\title{
Borylative Cyclization of 1,6-Allenynes Driven by $\mathrm{BCl}_{3}$
}

Chun-Hua Yang, ${ }^{\dagger}$ Xiangkun Sun, ${ }^{\dagger}$ Congcong Niu, ${ }^{\dagger}$ Zhiwei Zhang, $^{\dagger}$ Mingzhu Liu, ${ }^{\dagger}$ Fangjie

Zheng, ${ }^{\dagger}$ Ling Jiang, ${ }^{\dagger}$ Xiangtao Kong, ${ }^{*, \dagger}$ Zhantao Yang ${ }^{*},+$

${ }^{\dagger}$ Henan Key Laboratory of New Optoelectronic Functional Materials, College of Chemistry and Chemical Engineering, Anyang Normal University, 436 Xian'ge Road, Anyang 455000, People’s Republic of China

* State Key Laboratory of Molecular Reaction Dynamics, Dalian Institute of Chemical Physics, Chinese Academy of Sciences, Dalian 116023, People's Republic of 238 China

KEYWORDS Borylative Cyclization, $\mathrm{BCl}_{3}$, 1,6-Allenynes, Suzuki coupling 


\section{Table of Contents}

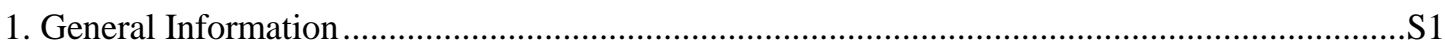

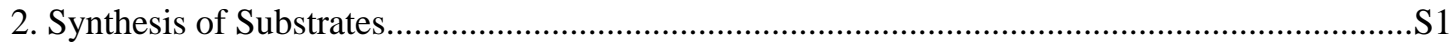

3. Typical Procedure for Borylative Cyclization.......................................................................... 11

4. Derivatization of Borylative Cyclization products.................................................................S22

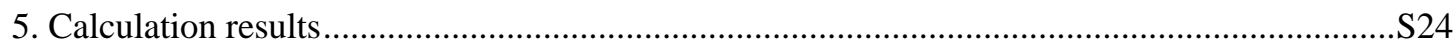

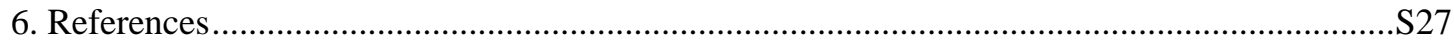

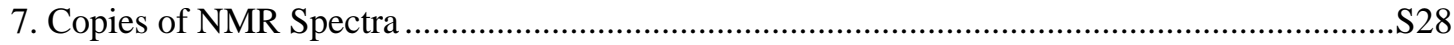

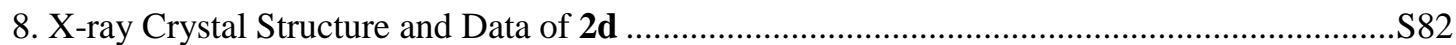

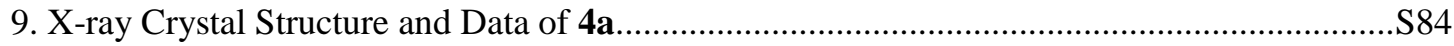




\section{General Information}

Unless otherwise noted, all reagents were used as received from commercial suppliers. Analytical thin layer chromatography (TLC) was performed on silica gel $\mathrm{GF}_{254}$. Visualization was accomplished by irradiation with UV light at $254 \mathrm{~nm}$ or $\mathrm{KMnO}_{4}$ stain solution. Column chromatography was performed on silica gel (200 - 300 mesh). ${ }^{1} \mathrm{H}$ NMR spectra were recorded on a Bruker DRX-400 spectrometer (400 MHz). Chemical shifts were reported in parts per million (ppm) referenced to $0.0 \mathrm{ppm}$ for tetramethylsilane. Data are reported as follows: chemical shift, multiplicity $(\mathrm{s}=$ singlet, $\mathrm{d}=$ doublet, $\mathrm{t}=$ triplet, $\mathrm{q}=$ quartet, $\mathrm{m}=$ multiplet, $\mathrm{br}=$ broad $)$, coupling constants (Hz) and integration. ${ }^{13} \mathrm{C}$ NMR spectra were recorded on a Bruker DRX-400 spectrometer $(100 \mathrm{MHz})$ and were fully decoupled by broad band proton decoupling. Chemical shifts were reported in parts per million (ppm) referenced to $77.16 \mathrm{ppm}$ for $\mathrm{CDCl}_{3} .{ }^{11} \mathrm{~B} \mathrm{NMR}$ Spectra were recorded on a Bruker DRX-400 spectrometer (128 MHz). High resolution mass spectra (HRMS) were recorded on a Waters LCT PremierxeTM (USA) (with Electron Spray Ionization as mass analyzer). Single-crystal experiments were recorded on Bruker Smart Apex II. Reactions that require heating were carried out with oil bath.

\section{Synthesis of Substrates}
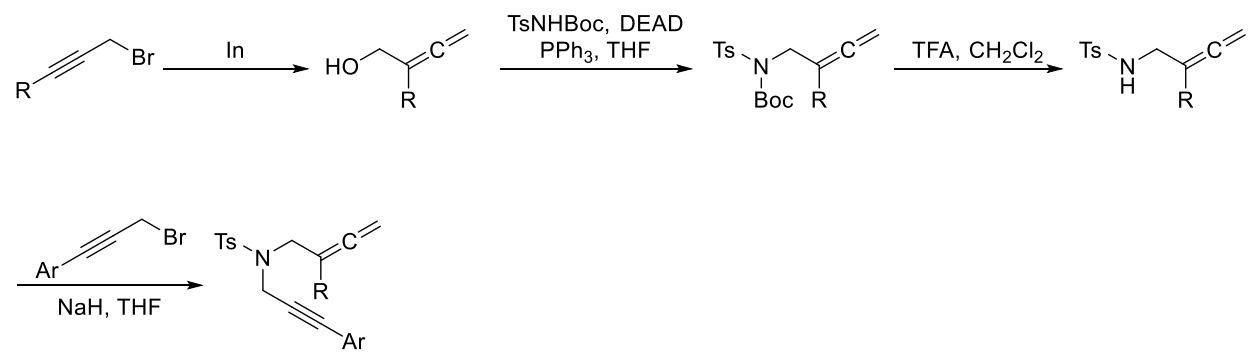

To a vigorously stirring mixture of propargyl bromide $(2.0 \mathrm{mmol}, 1.0$ equiv) and water at room temperature is added formaldehyde and indium ( $3.0 \mathrm{mmol}, 1.5$ equiv). After 12 hours, the reaction is extracted with $\mathrm{CH}_{2} \mathrm{Cl}_{2}$, and the organic layer dried $\left(\mathrm{Na}_{2} \mathrm{SO}_{4}\right)$ and concentrated. The residue was purified by column chromatography on silica gel to give buta-2,3-dien-1-ol.

To a solution of $\mathrm{PPh}_{3}$ (1.5 equiv) in THF was added DEAD (1.5 equiv) at $-15^{\circ} \mathrm{C}$, and the mixture was stirred at the same temperature for $15 \mathrm{~min}$. To the mixture was added alcohol (1.0 equiv) in 
THF at $-15^{\circ} \mathrm{C}$, and the mixture was stirred at the same temperature for $15 \mathrm{~min}$. To the mixture was added TsNHBoc ( 1.5 equiv) in THF at $-15^{\circ} \mathrm{C}$, and the mixture was stirred at the same temperature for $1 \mathrm{~h}$, and at room temperature overnight. After removal of THF, the residue was purified by column chromatography on silica gel to give tert-butyl buta-2,3-dien-1-yl(tosyl)carbamate.

To a solution of tert-butyl buta-2,3-dien-1-yl(tosyl)carbamate (1.0 equiv) in $\mathrm{CH}_{2} \mathrm{Cl}_{2}$ was added TFA (1.5 equiv) at room temperature, and the mixture was stirred for $30 \mathrm{~min}$. The reaction was quenched with Sat. $\mathrm{NaHCO}_{3}$ and then extracted with $\mathrm{CH}_{2} \mathrm{Cl}_{2}$. The organic layers were dried over $\mathrm{Na}_{2} \mathrm{SO}_{4}$, filtered and evaporated under reduced pressure. The crude product was purified by column chromatography on silica gel.

To a solution of N-(buta-2,3-dien-1-yl)-4-methylbenzenesulfonamide (1.0 equiv) in anhydrous THF was added $\mathrm{NaH}$ (1.3 equiv) with care and the mixture was stirred for 30 minutes. Propargyl bromide ((1.5 equiv)) was added and the reaction was stirred for $1 \mathrm{~h}$. It was then quenched with water and the product was extracted with EtOAc. The organic extracts were dried over $\mathrm{Na}_{2} \mathrm{SO}_{4}$ and then concentrated in vacuo. The crude product was purified by column chromatography on silica gel.

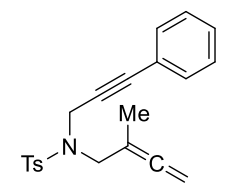

\section{4-methyl-N-(2-methylbuta-2,3-dien-1-yl)-N-(3-phenylprop-2-yn-1-yl)benzenesulfonamide}

(1a)

Compound 1a was prepared according to the general procedure from 1-bromobut-2-yne (265 mg, $2 \mathrm{mmol}$ ) and was isolated as a yellow oil (280 $\mathrm{mg}, 40 \%$ total yield) after short column chromatography (5\%-10\% EtOAc/Petroleum). ${ }^{1} \mathrm{H}$ NMR (400 MHz, $\left.\mathrm{CDCl}_{3}\right) \delta 7.76$ (d, $J=8.2 \mathrm{~Hz}$, 2H), $7.24(\mathrm{dt}, J=7.6,2.7 \mathrm{~Hz}, 5 \mathrm{H}), 7.04(\mathrm{~d}, J=6.8 \mathrm{~Hz}, 2 \mathrm{H}), 4.69(\mathrm{dd}, J=5.2,2.5 \mathrm{~Hz}, 2 \mathrm{H}), 4.31(\mathrm{~s}$, 2H), $3.84(\mathrm{~s}, 2 \mathrm{H}), 2.31(\mathrm{~s}, 3 \mathrm{H}), 1.76(\mathrm{t}, J=3.1 \mathrm{~Hz}, 3 \mathrm{H}) .{ }^{13} \mathrm{C} \mathrm{NMR}\left(100 \mathrm{MHz}, \mathrm{CDCl}_{3}\right) \delta 207.8$, $143.5,136.0,131.5,129.5,128.3,128.1,127.8,122.3,93.4,85.6,81.5,75.1,50.2,36.5,21.4,15.9$. HRMS-ESI (m/z): $[\mathrm{M}+\mathrm{H}]^{+}$calcd for $\mathrm{C}_{21} \mathrm{H}_{22} \mathrm{NO}_{2} \mathrm{~S}, 352.1371$; found: 352.1384 .

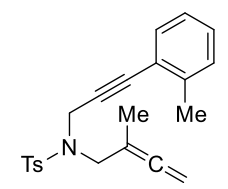




\section{4-methyl-N-(2-methylbuta-2,3-dien-1-yl)-N-(3-(o-tolyl)prop-2-yn-1-yl)benzenesulfonamide}

(1b)

Compound $1 \mathbf{b}$ was prepared according to the general procedure from 1-bromobut-2-yne (266 mg, $2 \mathrm{mmol}$ ) and was isolated as a yellow oil (275 mg, 38\% total yield) after short column chromatography (5\%-10\% EtOAc/Petroleum). ${ }^{1} \mathrm{H}$ NMR (400 MHz, $\left.\mathrm{CDCl}_{3}\right) \delta 7.64$ (d, J=7.8 Hz, 2H), $7.11-6.85(\mathrm{~m}, 6 \mathrm{H}), 4.57$ (s, 2H), $4.26(\mathrm{~s}, 2 \mathrm{H}), 3.77$ (s, 2H), 2.13 (s, 3H), 2.03 (s, 3H), 1.66 $(\mathrm{d}, J=2.3 \mathrm{~Hz}, 3 \mathrm{H}) .{ }^{13} \mathrm{C} \mathrm{NMR}\left(100 \mathrm{MHz}, \mathrm{CDCl}_{3}\right) \delta 207.7,143.5,139.9,136.0,131.9,129.4,129.2$, 128.3, 127.6, 125.3, 122.1, 93.3, 85.3, 84.5, 75.1, 50.1, 36.5, 21.3, 20.4, 15.8. HRMS-ESI (m/z): $[\mathrm{M}+\mathrm{H}]^{+}$calcd for $\mathrm{C}_{22} \mathrm{H}_{24} \mathrm{NO}_{2} \mathrm{~S}, 366.1528$; found: 366.1524 .

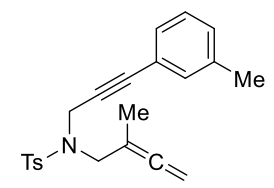

\section{4-methyl-N-(2-methylbuta-2,3-dien-1-yl)-N-(3-(m-tolyl)prop-2-yn-1-yl)benzenesulfonamide}

(1c)

Compound 1c was prepared according to the general procedure from 1-bromobut-2-yne (265 mg, $2 \mathrm{mmol})$ and was isolated as a yellow oil (250 $\mathrm{mg}, 34 \%$ total yield) after short column chromatography (5\%-10\% EtOAc/Petroleum). ${ }^{1} \mathrm{H}$ NMR (400 MHz, $\left.\mathrm{CDCl}_{3}\right) \delta 7.65$ (d, $J=7.8 \mathrm{~Hz}$, 2H), $7.11(\mathrm{~d}, J=7.7 \mathrm{~Hz}, 2 \mathrm{H}), 7.03-6.89(\mathrm{~m}, 2 \mathrm{H}), 6.78-6.69(\mathrm{~m}, 2 \mathrm{H}), 4.58(\mathrm{~s}, 2 \mathrm{H}), 4.20(\mathrm{~s}, 2 \mathrm{H})$, $3.74(\mathrm{~s}, 2 \mathrm{H}), 2.19$ (s, 3H), 2.15 (s, 3H), 1.64 (d, $J=2.4 \mathrm{~Hz}, 3 \mathrm{H}) .{ }^{13} \mathrm{C} \mathrm{NMR}\left(100 \mathrm{MHz}, \mathrm{CDCl}_{3}\right) \delta$ 207.6, 143.3, 137.6, 136.0, 131.9, 129.4, 129.1, 128.5, 127.9, 127.7, 122.0, 93.3, 85.7, 81.0, 75.1, 50.1, 36.5, 21.3, 21.1, 15.8. HRMS-ESI (m/z): $[\mathrm{M}+\mathrm{H}]^{+}$calcd for $\mathrm{C}_{22} \mathrm{H}_{24} \mathrm{NO}_{2} \mathrm{~S}, 366.1528$; found: 366.1534 .

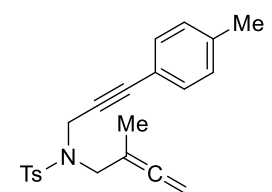

\section{4-methyl-N-(2-methylbuta-2,3-dien-1-yl)-N-(3-(p-tolyl)prop-2-yn-1-yl)benzenesulfonamide} (1d)

Compound 1d was prepared according to the general procedure from 1-bromobut-2-yne (265 mg, $2 \mathrm{mmol}$ ) and was isolated as a yellow oil $(260 \mathrm{mg}, 36 \%$ total yield) after short column chromatography (5\%-10\% EtOAc/Petroleum). ${ }^{1} \mathrm{H}$ NMR (400 MHz, $\left.\mathrm{CDCl}_{3}\right) \delta 7.61$ (d, J=8.3 Hz, 
2H), $7.05(\mathrm{~d}, J=8.1 \mathrm{~Hz}, 2 \mathrm{H}), 6.86(\mathrm{~d}, J=8.0 \mathrm{~Hz}, 2 \mathrm{H}), 6.79(\mathrm{~d}, J=8.1 \mathrm{~Hz}, 2 \mathrm{H}), 4.63-4.40(\mathrm{~m}$, $2 \mathrm{H}), 4.16(\mathrm{~s}, 2 \mathrm{H}), 3.71(\mathrm{~s}, 2 \mathrm{H}), 2.13(\mathrm{~s}, 6 \mathrm{H}), 1.61(\mathrm{t}, J=3.1 \mathrm{~Hz}, 3 \mathrm{H}) .{ }^{13} \mathrm{C}$ NMR $\left(100 \mathrm{MHz}, \mathrm{CDCl}_{3}\right)$ $\delta 207.5,143.2,138.1,135.9,131.2,129.3,128.6,127.5,119.0,93.2,85.5,80.5,74.9,50.0,36.3$, 21.1, 21.1, 15.6. HRMS-ESI (m/z): $[\mathrm{M}+\mathrm{H}]^{+}$calcd for $\mathrm{C}_{22} \mathrm{H}_{24} \mathrm{NO}_{2} \mathrm{~S}, 366.1528$; found: 366.1543 .

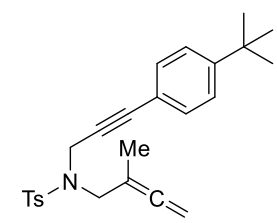

N-(3-(4-(tert-butyl)phenyl)prop-2-yn-1-yl)-4-methyl-N-(2-methylbuta-2,3-dien-1-yl)benzenes ulfonamide (1e)

Compound 1e was prepared according to the general procedure from 1-bromobut-2-yne (265 mg, $2 \mathrm{mmol})$ and was isolated as a yellow oil $(290 \mathrm{mg}, 36 \%$ total yield) after short column chromatography (5\%-10\% EtOAc/Petroleum). ${ }^{1} \mathrm{H}$ NMR (400 MHz, $\left.\mathrm{CDCl}_{3}\right) \delta 7.65$ (d, $J=8.1 \mathrm{~Hz}$, 2H), $7.12(\mathrm{~d}, J=8.4 \mathrm{~Hz}, 2 \mathrm{H}), 7.09(\mathrm{~d}, J=8.3 \mathrm{~Hz}, 2 \mathrm{H}), 6.88(\mathrm{~d}, J=8.3 \mathrm{~Hz}, 2 \mathrm{H}), 4.57(\mathrm{~d}, J=2.5$ $\mathrm{Hz}, 2 \mathrm{H}), 4.19$ (s, 2H), 3.74 (s, 2H), 2.16 (s, 3H), 1.64 (t, $J=2.9 \mathrm{~Hz}, 3 \mathrm{H}), 1.16$ (s, 9H). ${ }^{13} \mathrm{C}$ NMR $\left(100 \mathrm{MHz}, \mathrm{CDCl}_{3}\right) \delta$ 207.7, 151.4, 143.3, 136.0, 131.2, 129.4, 127.6, 124.9, 119.2, 93.3, 85.7, $80.7, \quad 75.0, \quad 50.1, \quad 36.5, \quad 34.6, \quad 31.0, \quad 21.3, \quad 15.8$. HRMS-ESI $(\mathrm{m} / \mathrm{z}):[\mathrm{M}+\mathrm{Na}]^{+}$calcd for $\mathrm{C}_{25} \mathrm{H}_{29} \mathrm{NNaO}_{2} \mathrm{~S}$, 430.1817; found: 430.1826 .

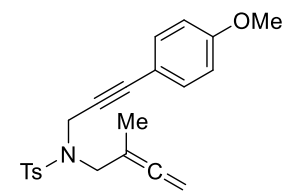

N-(3-(4-methoxyphenyl)prop-2-yn-1-yl)-4-methyl-N-(2-methylbuta-2,3-dien-1-yl)benzenesulf onamide (1f)

Compound 1f was prepared according to the general procedure from 1-bromobut-2-yne (265 mg, $2 \mathrm{mmol})$ and was isolated as a yellow oil $(190 \mathrm{mg}, 25 \%$ total yield) after short column chromatography (5\%-10\% EtOAc/Petroleum). ${ }^{1} \mathrm{H}$ NMR (400 MHz, $\left.\mathrm{CDCl}_{3}\right) \delta 7.76$ (d, $J=8.2 \mathrm{~Hz}$, 2H), $7.24(\mathrm{~d}, J=8.2 \mathrm{~Hz}, 2 \mathrm{H}), 6.99(\mathrm{~d}, J=8.7 \mathrm{~Hz}, 2 \mathrm{H}), 6.75(\mathrm{~d}, J=8.7 \mathrm{~Hz}, 2 \mathrm{H}), 4.69(\mathrm{~d}, J=2.7$ $\mathrm{Hz}, 2 \mathrm{H}), 4.29$ (s, 2H), 3.83 (s, 2H), 3.78 (s, 3H), 2.33 (s, 3H), 1.75 (t, $J=3.1 \mathrm{~Hz}, 3 \mathrm{H}) .{ }^{13} \mathrm{C}$ NMR $\left(100 \mathrm{MHz}, \mathrm{CDCl}_{3}\right) \delta 207.8,159.6,143.4,136.1,133.0,129.5,127.8,114.4,113.7,93.5,85.5,80.0$, 75.1, 55.3, 50.2, 36.6, 21.4, 15.9. HRMS-ESI (m/z): $[\mathrm{M}+\mathrm{H}]^{+}$calcd for $\mathrm{C}_{22} \mathrm{H}_{24} \mathrm{NO}_{3} \mathrm{~S}, 382.1477$; found: 382.1463 . 


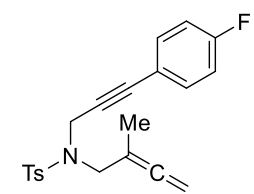

N-(3-(4-fluorophenyl)prop-2-yn-1-yl)-4-methyl-N-(2-methylbuta-2,3-dien-1-yl)benzenesulfon amide (1g)

Compound $1 \mathrm{~g}$ was prepared according to the general procedure from 1-bromobut-2-yne (265 mg, $2 \mathrm{mmol}$ ) and was isolated as a yellow oil (300 $\mathrm{mg}, 41 \%$ total yield) after short column chromatography (5\%-10\% EtOAc/Petroleum). ${ }^{1} \mathrm{H}$ NMR $\left(400 \mathrm{MHz}, \mathrm{CDCl}_{3}\right) \delta 7.76(\mathrm{~d}, J=8.2 \mathrm{~Hz}$, 2H), $7.24(\mathrm{~d}, J=8.1 \mathrm{~Hz}, 2 \mathrm{H}), 7.10-7.00(\mathrm{~m}, 2 \mathrm{H}), 6.92(\mathrm{t}, J=8.7 \mathrm{~Hz}, 2 \mathrm{H}), 4.69(\mathrm{dd}, J=5.2,2.3$ $\mathrm{Hz}, 2 \mathrm{H}), 4.29(\mathrm{~s}, 2 \mathrm{H}), 3.84(\mathrm{~s}, 2 \mathrm{H}), 2.33(\mathrm{~s}, 3 \mathrm{H}), 1.75(\mathrm{t}, J=3.1 \mathrm{~Hz}, 3 \mathrm{H}) .{ }^{13} \mathrm{C} \mathrm{NMR}(100 \mathrm{MHz}$, $\left.\mathrm{CDCl}_{3}\right) \delta 207.9,143.5,136.2,133.6,133.5,129.6,127.9,115.6,115.4,93.5,84.6,81.4,75.2,50.4$, 36.6, 21.5, 16.0. HRMS-ESI (m/z): $[\mathrm{M}+\mathrm{H}]^{+}$calcd for $\mathrm{C}_{21} \mathrm{H}_{21} \mathrm{FNO}_{2} \mathrm{~S}, 370.1277$; found: 370.1292 .

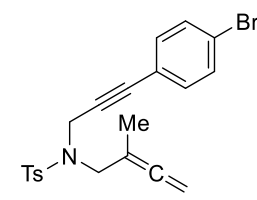

N-(3-(4-bromophenyl)prop-2-yn-1-yl)-4-methyl-N-(2-methylbuta-2,3-dien-1-yl)benzenesulfo namide (1h)

Compound 1h was prepared according to the general procedure from 1-bromobut-2-yne (265 mg, $2 \mathrm{mmol}$ ) and was isolated as a yellow oil (320 mg, 37\% total yield) after short column chromatography (5\%-10\% EtOAc/Petroleum). ${ }^{1} \mathrm{H}$ NMR $\left(400 \mathrm{MHz}, \mathrm{CDCl}_{3}\right) \delta 7.75(\mathrm{~d}, J=8.2 \mathrm{~Hz}$, 2H), $7.36(\mathrm{~d}, J=8.4 \mathrm{~Hz}, 2 \mathrm{H}), 7.24(\mathrm{~d}, J=8.2 \mathrm{~Hz}, 2 \mathrm{H}), 6.90(\mathrm{~d}, J=8.4 \mathrm{~Hz}, 2 \mathrm{H}), 4.68(\mathrm{dd}, J=5.2$, $2.4 \mathrm{~Hz}, 2 \mathrm{H}), 4.29(\mathrm{~s}, 2 \mathrm{H}), 3.83(\mathrm{t}, J=1.9 \mathrm{~Hz}, 2 \mathrm{H}), 2.33(\mathrm{~s}, 3 \mathrm{H}), 1.75(\mathrm{t}, J=3.1 \mathrm{~Hz}, 3 \mathrm{H}) .{ }^{13} \mathrm{C} \mathrm{NMR}$ $\left(100 \mathrm{MHz}, \mathrm{CDCl}_{3}\right) \delta 207.8,143.5,136.0,132.9,131.3,129.5,127.8,122.6,121.2,93.3,84.5$, 82.8, 75.1, 50.3, 36.5, 21.5, 15.8. HRMS-ESI $(\mathrm{m} / \mathrm{z})$ : $[\mathrm{M}+\mathrm{Na}]^{+}$calcd for $\mathrm{C}_{21} \mathrm{H}_{20} \mathrm{BrNNaO}_{2} \mathrm{~S}$, 452.0296; found: 452.0279 .

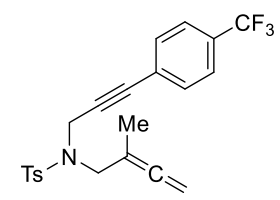

4-methyl-N-(2-methylbuta-2,3-dien-1-yl)-N-(3-(4-(trifluoromethyl)phenyl)prop-2-yn-1-yl)ben zenesulfonamide (1i)

Compound 1i was prepared according to the general procedure from 1-bromobut-2-yne (265 mg, 2 
mmol) and was isolated as a yellow oil (280 $\mathrm{mg}, 33 \%$ total yield) after short column chromatography (5\%-10\% EtOAc/Petroleum). ${ }^{1} \mathrm{H}$ NMR (400 MHz, $\left.\mathrm{CDCl}_{3}\right) \delta 7.76$ (d, $J=8.3 \mathrm{~Hz}$, 2H), $7.49(\mathrm{~d}, J=8.1 \mathrm{~Hz}, 2 \mathrm{H}), 7.24(\mathrm{~d}, J=8.0 \mathrm{~Hz}, 2 \mathrm{H}), 7.14(\mathrm{~d}, J=8.0 \mathrm{~Hz}, 2 \mathrm{H}), 4.74-4.62(\mathrm{~m}$, 2H), $4.32(\mathrm{~s}, 2 \mathrm{H}), 3.85(\mathrm{t}, J=2.1 \mathrm{~Hz}, 2 \mathrm{H}), 2.31(\mathrm{~s}, 3 \mathrm{H}), 1.76(\mathrm{t}, J=3.1 \mathrm{~Hz}, 3 \mathrm{H}) .{ }^{13} \mathrm{C}$ NMR $(100$ $\left.\mathrm{MHz}, \mathrm{CDCl}_{3}\right) \delta 208.0,143.7,136.2,131.9,129.6,127.9,125.2,125.2,125.1,125.1,93.5,84.4$, 84.3, 75.3, 50.6, 36.5, 21.5, 16.0. HRMS-ESI (m/z): $[\mathrm{M}+\mathrm{H}]^{+}$calcd for $\mathrm{C}_{22} \mathrm{H}_{21} \mathrm{~F}_{3} \mathrm{NO}_{2} \mathrm{~S}, 420.1245$; found: 420.1225 .

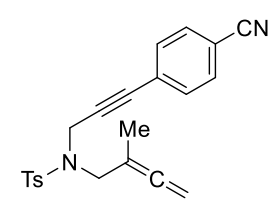

N-(3-(4-cyanophenyl)prop-2-yn-1-yl)-4-methyl-N-(2-methylbuta-2,3-dien-1-yl)benzenesulfon amide (1j)

Compound $\mathbf{1} \mathbf{j}$ was prepared according to the general procedure from 1-bromobut-2-yne (265 mg, $2 \mathrm{mmol})$ and was isolated as a yellow oil (220 mg, $29 \%$ total yield) after short column chromatography (5\%-10\% EtOAc/Petroleum). ${ }^{1} \mathrm{H}$ NMR (400 MHz, $\left.\mathrm{CDCl}_{3}\right) \delta 7.68(\mathrm{~d}, J=6.9 \mathrm{~Hz}$, 2H), $7.45(\mathrm{~d}, J=6.9 \mathrm{~Hz}, 2 \mathrm{H}), 7.17(\mathrm{~d}, J=7.6 \mathrm{~Hz}, 2 \mathrm{H}), 7.05(\mathrm{~d}, J=7.0 \mathrm{~Hz}, 2 \mathrm{H}), 4.61(\mathrm{~s}, 2 \mathrm{H}), 4.25$ (s, 2H), 3.76 (s, 2H), 2.24 (s, 3H), 1.67 (d, $J=2.1 \mathrm{~Hz}, 3 \mathrm{H}) .{ }^{13} \mathrm{C} \mathrm{NMR}\left(100 \mathrm{MHz}, \mathrm{CDCl}_{3}\right) \delta 207.9$, $143.6,136.0,132.1,131.9,129.6,127.8,127.2,118.3,111.8,93.3,86.5,84.0,75.2,50.6,36.5$, 21.5, 15.9. HRMS-ESI (m/z): [M+H] $]^{+}$calcd for $\mathrm{C}_{22} \mathrm{H}_{21} \mathrm{~N}_{2} \mathrm{O}_{2} \mathrm{~S}, 377.1324$; found: 377.1346 .

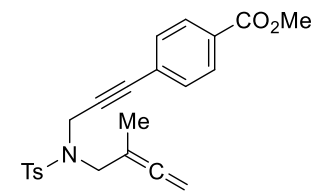

methyl 4-(3-((4-methyl-N-(2-methylbuta-2,3-dien-1-yl)phenyl)sulfonamido)prop-1-yn-1-yl) benzoate (1k)

Compound 1k was prepared according to the general procedure from 1-bromobut-2-yne (265 mg, $2 \mathrm{mmol})$ and was isolated as a yellow oil (250 $\mathrm{mg}, 31 \%$ total yield) after short column chromatography (5\%-10\% EtOAc/Petroleum). ${ }^{1} \mathrm{H}$ NMR (400 MHz, $\left.\mathrm{CDCl}_{3}\right) \delta 7.90$ (d, $J=8.3 \mathrm{~Hz}$, 2H), $7.76(\mathrm{~d}, J=8.2 \mathrm{~Hz}, 2 \mathrm{H}), 7.24(\mathrm{~d}, J=8.1 \mathrm{~Hz}, 2 \mathrm{H}), 7.09(\mathrm{~d}, J=8.3 \mathrm{~Hz}, 2 \mathrm{H}), 4.69$ (d, $J=2.8$ $\mathrm{Hz}, 2 \mathrm{H}), 4.33$ (s, 2H), 3.91 (s, 3H), 3.85 (s, 2H), 2.31 (s, 3H), 1.76 (t, $J=3.1 \mathrm{~Hz}, 3 \mathrm{H}) .{ }^{13} \mathrm{C}$ NMR $\left(100 \mathrm{MHz}, \mathrm{CDCl}_{3}\right) \delta 207.9,166.4,143.6,136.0,131.5,129.7,129.6,129.3,127.8,127.0,93.4$, 
84.9, 84.8, 75.2, 52.3, 50.5, 36.5, 21.5, 15.9. HRMS-ESI (m/z): $[\mathrm{M}+\mathrm{H}]^{+}$calcd for $\mathrm{C}_{23} \mathrm{H}_{24} \mathrm{NO}_{4} \mathrm{~S}$, 410.1426; found: 410.1412 .

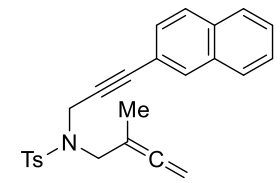

4-methyl-N-(2-methylbuta-2,3-dien-1-yl)-N-(3-(naphthalen-2-yl)prop-2-yn-1-yl)benzenesulfo namide (11)

Compound 11 was prepared according to the general procedure from 1-bromobut-2-yne (265 mg, 2 mmol) and was isolated as a yellow oil (320 mg, $40 \%$ total yield) after short column chromatography (5\%-10\% EtOAc/Petroleum). ${ }^{1} \mathrm{H}$ NMR (400 MHz, $\left.\mathrm{CDCl}_{3}\right) \delta 7.74$ (d, $J=7.7 \mathrm{~Hz}$, 1H), $7.67-7.58(\mathrm{~m}, 4 \mathrm{H}), 7.39-7.25(\mathrm{~m}, 2 \mathrm{H}), 7.16(\mathrm{q}, J=7.2 \mathrm{~Hz}, 2 \mathrm{H}), 6.93(\mathrm{~d}, J=7.7 \mathrm{~Hz}, 2 \mathrm{H})$, $4.59(\mathrm{~s}, 2 \mathrm{H}), 4.35$ (s, 2H), $3.82(\mathrm{~s}, 2 \mathrm{H}), 1.91$ (s, 3H), $1.66(\mathrm{~s}, 3 \mathrm{H}) .{ }^{13} \mathrm{C} \mathrm{NMR}\left(100 \mathrm{MHz}, \mathrm{CDCl}_{3}\right) \delta$ 207.8, 143.5, 135.9, 132.9, 130.5, 129.5, 128.8, 128.1, 127.6, 126.6, 126.3, 125.9, 124.9, 119.9, 93.4, 86.3, 83.8, 75.2, 50.3, 36.7, 21.1, 15.8. HRMS-ESI (m/z): $[\mathrm{M}+\mathrm{H}]^{+}$calcd for $\mathrm{C}_{25} \mathrm{H}_{24} \mathrm{NO}_{2} \mathrm{~S}$, 402.1528; found: 402.1539 .

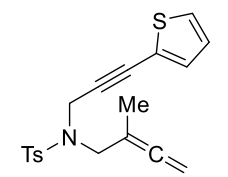

4-methyl-N-(2-methylbuta-2,3-dien-1-yl)-N-(3-(thiophen-2-yl)prop-2-yn-1-yl)benzenesulfona mide (1m)

Compound 1m was prepared according to the general procedure from 1-bromobut-2-yne (265 mg, $2 \mathrm{mmol})$ and was isolated as a yellow oil $(250 \mathrm{mg}, 35 \%$ total yield) after short column chromatography (5\%-10\% EtOAc/Petroleum). ${ }^{1} \mathrm{H}$ NMR (400 MHz, $\left.\mathrm{CDCl}_{3}\right) \delta 7.67$ (d, J=8.2 Hz, 2H), $7.18(\mathrm{~d}, J=8.1 \mathrm{~Hz}, 2 \mathrm{H}), 7.10(\mathrm{dd}, J=4.5,1.5 \mathrm{~Hz}, 1 \mathrm{H}), 6.88-6.75(\mathrm{~m}, 2 \mathrm{H}), 4.62(\mathrm{~d}, J=2.7$ $\mathrm{Hz}, 2 \mathrm{H}), 4.24$ (s, 2H), 3.73 (s, 2H), 2.27 (s, 3H), 1.67 (t, $J=3.0 \mathrm{~Hz}, 3 \mathrm{H}) .{ }^{13} \mathrm{C}$ NMR (100 MHz, $\left.\mathrm{CDCl}_{3}\right) \delta 207.8,143.6,135.9,132.2,129.6,127.7,127.2,126.7,122.2,93.4,85.6,78.9,75.2,50.3$, 36.7, 21.5, 15.9. HRMS-ESI (m/z): [M+H] $]^{+}$calcd for $\mathrm{C}_{19} \mathrm{H}_{20} \mathrm{NO}_{2} \mathrm{~S}_{2}, 358.0935$; found: 358.0929 .

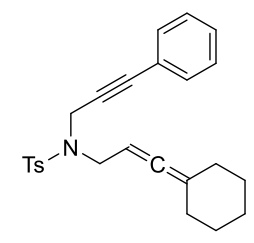




\section{N-(3-cyclohexylideneallyl)-4-methyl-N-(3-phenylprop-2-yn-1-yl)benzenesulfonamide (1n)}

Compound 1n was prepared according to the general procedure from 1-bromobut-2-yne (265 mg, $2 \mathrm{mmol})$ and was isolated as a yellow oil $(190 \mathrm{mg}, 23 \%$ total yield) after short column chromatography (5\%-10\% EtOAc/Petroleum). ${ }^{1} \mathrm{H}$ NMR (400 MHz, $\left.\mathrm{CDCl}_{3}\right) \delta 7.77$ (d, $J=8.2 \mathrm{~Hz}$, 2H), $7.27-7.22(\mathrm{~m}, 5 \mathrm{H}), 7.05(\mathrm{dd}, J=7.9,1.3 \mathrm{~Hz}, 2 \mathrm{H}), 5.03-4.75(\mathrm{~m}, 1 \mathrm{H}), 4.38(\mathrm{~s}, 2 \mathrm{H}), 3.86(\mathrm{~d}$ $J=7.0 \mathrm{~Hz}, 2 \mathrm{H}), 2.32(\mathrm{~s}, 3 \mathrm{H}), 2.18-2.00(\mathrm{~m}, 4 \mathrm{H}), 1.59-1.34(\mathrm{~m}, 6 \mathrm{H}) .{ }^{13} \mathrm{C}$ NMR $(100 \mathrm{MHz}$, $\left.\mathrm{CDCl}_{3}\right) \delta 200.8,143.4,136.1,131.5,129.5,128.3,128.1,127.8,122.4,104.2,85.4,83.5,81.8$, 47.1, 36.4, 31.3, 27.2, 25.9, 21.4. HRMS-ESI (m/z): $[\mathrm{M}+\mathrm{H}]^{+}$calcd for $\mathrm{C}_{25} \mathrm{H}_{28} \mathrm{NO}_{2} \mathrm{~S}, 406.1841$; found: 406.1867.

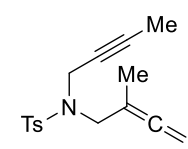

\section{N-(but-2-yn-1-yl)-4-methyl-N-(2-methylbuta-2,3-dien-1-yl)benzenesulfonamide (10)}

Compound 10 was prepared according to the general procedure from 1-bromobut-2-yne (265 mg, $2 \mathrm{mmol})$ and was isolated as a yellow oil (115 $\mathrm{mg}, 20 \%$ total yield) after short column chromatography (5\%-10\% EtOAc/Petroleum). ${ }^{1} \mathrm{H}$ NMR (400 MHz, $\left.\mathrm{CDCl}_{3}\right) \delta 7.73$ (d, J=8.3 Hz, 2H), $7.29(\mathrm{~d}, J=8.9 \mathrm{~Hz}, 2 \mathrm{H}), 4.75-4.59(\mathrm{~m}, 2 \mathrm{H}), 4.03(\mathrm{q}, J=2.3 \mathrm{~Hz}, 2 \mathrm{H}), 3.75(\mathrm{t}, J=2.3 \mathrm{~Hz}$, 2H), $2.42(\mathrm{~s}, 3 \mathrm{H}), 1.71(\mathrm{t}, J=3.2 \mathrm{~Hz}, 3 \mathrm{H}), 1.50(\mathrm{t}, J=2.4 \mathrm{~Hz}, 3 \mathrm{H}) .{ }^{13} \mathrm{C} \mathrm{NMR}\left(100 \mathrm{MHz}, \mathrm{CDCl}_{3}\right) \delta$ 207.6, 143.3, 136.3, 129.3, 127.9, 93.7, 81.5, 75.1, 71.5, 49.9, 36.2, 21.6, 15.9, 3.3. Spectral data was

consistent with

N-(but-2-yn-1-yl)-4-methyl-N-(2-methylbuta-2,3-dien-1-yl)benzenesulfonamide. ${ }^{1}$

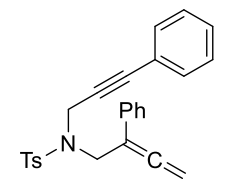

4-methyl-N-(2-phenylbuta-2,3-dien-1-yl)-N-(3-phenylprop-2-yn-1-yl)benzenesulfonamide (3a)

Compound 3a was prepared according to the general procedure from (3-bromoprop-1-yn-1-yl)benzene (390 mg, 2 mmol) and was isolated as a yellow oil (340 mg, 41\% total yield) after short column chromatography (5\%-10\% EtOAc/Petroleum). ${ }^{1} \mathrm{H}$ NMR (400 MHz, $\left.\mathrm{CDCl}_{3}\right) \delta 7.80(\mathrm{~d}, J=7.3 \mathrm{~Hz}, 2 \mathrm{H}), 7.59(\mathrm{~d}, J=7.7 \mathrm{~Hz}, 2 \mathrm{H}), 7.34(\mathrm{t}, J=7.3 \mathrm{~Hz}, 2 \mathrm{H}), 7.28-7.15$ $(\mathrm{m}, 6 \mathrm{H}), 7.02(\mathrm{~d}, J=7.4 \mathrm{~Hz}, 2 \mathrm{H}), 5.13(\mathrm{~s}, 2 \mathrm{H}), 4.35(\mathrm{~s}, 2 \mathrm{H}), 4.27$ (s, 2H), 2.30 (s, 3H). ${ }^{13} \mathrm{C}$ NMR 
$\left(100 \mathrm{MHz}, \mathrm{CDCl}_{3}\right) \delta 210.3,143.7,135.4,133.3,131.5,129.6,128.7,128.4,128.2,128.1,127.4$, 126.6, 122.4, 99.7, 86.1, 81.3, 78.9, 47.4, 36.6, 21.5. HRMS-ESI $(\mathrm{m} / \mathrm{z}):[\mathrm{M}+\mathrm{H}]^{+}$calcd for $\mathrm{C}_{26} \mathrm{H}_{24} \mathrm{NO}_{2} \mathrm{~S}, 414.1528$; found: 414.1552 .

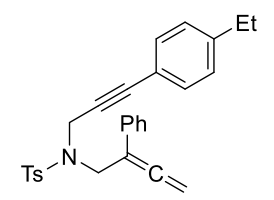

N-(3-(4-ethylphenyl)prop-2-yn-1-yl)-4-methyl-N-(2-phenylbuta-2,3-dien-1-yl)benzenesulfona mide (3b)

Compound 3b was prepared according to the general procedure from (3-bromoprop-1-yn-1-yl)benzene (390 mg, 2 mmol) and was isolated as a yellow oil (350 mg, 40\% total yield) after short column chromatography (5\%-10\% EtOAc/Petroleum). ${ }^{1} \mathrm{H}$ NMR (400 MHz, $\left.\mathrm{CDCl}_{3}\right) \delta 7.80(\mathrm{~d}, J=8.2 \mathrm{~Hz}, 2 \mathrm{H}), 7.59(\mathrm{~d}, J=7.5 \mathrm{~Hz}, 2 \mathrm{H}), 7.35(\mathrm{t}, J=7.7 \mathrm{~Hz}, 2 \mathrm{H}), 7.29-7.21$ (m, 3H), 7.05 (d, J=8.1 Hz, 2H), $6.94(\mathrm{~d}, J=8.1 \mathrm{~Hz}, 2 \mathrm{H}), 5.13(\mathrm{~s}, 2 \mathrm{H}), 4.35$ (s, 2H), 4.27 (s, 2H), $2.61(\mathrm{q}, J=7.6 \mathrm{~Hz}, 2 \mathrm{H}), 2.32(\mathrm{~s}, 3 \mathrm{H}), 1.20(\mathrm{t}, J=7.6 \mathrm{~Hz}, 3 \mathrm{H}) .{ }^{13} \mathrm{C} \mathrm{NMR}\left(100 \mathrm{MHz}, \mathrm{CDCl}_{3}\right) \delta$ $210.3,144.8,143.6,135.4,133.3,131.5,129.6,128.6,128.0,127.7,127.3,126.5,119.5,99.7$, 86.2, 80.5, 78.8, 47.2, 36.6, 28.8, 21.5, 15.4. HRMS-ESI (m/z): $[\mathrm{M}+\mathrm{H}]^{+}$calcd for $\mathrm{C}_{28} \mathrm{H}_{28} \mathrm{NO}_{2} \mathrm{~S}$, 442.1841; found: 442.1857 .

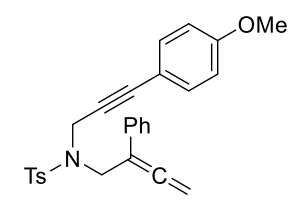

N-(3-(4-methoxyphenyl)prop-2-yn-1-yl)-4-methyl-N-(2-phenylbuta-2,3-dien-1-yl)benzenesulf onamide (3c)

Compound 3c was prepared according to the general procedure from (3-bromoprop-1-yn-1-yl)benzene (390 mg, 2 mmol) and was isolated as a yellow oil (260 mg, 29\% total yield) after short column chromatography (5\%-10\% EtOAc/Petroleum). ${ }^{1} \mathrm{H}$ NMR (400 MHz, $\left.\mathrm{CDCl}_{3}\right) \delta 7.80(\mathrm{~d}, J=8.1 \mathrm{~Hz}, 2 \mathrm{H}), 7.58(\mathrm{~d}, J=7.6 \mathrm{~Hz}, 2 \mathrm{H}), 7.35(\mathrm{t}, J=7.6 \mathrm{~Hz}, 2 \mathrm{H}), 7.25(\mathrm{t}, J=$ $6.7 \mathrm{~Hz}, 3 \mathrm{H}), 6.97(\mathrm{~d}, J=8.7 \mathrm{~Hz}, 2 \mathrm{H}), 6.75(\mathrm{~d}, J=8.7 \mathrm{~Hz}, 2 \mathrm{H}), 5.14(\mathrm{~s}, 2 \mathrm{H}), 4.34(\mathrm{~s}, 2 \mathrm{H}), 4.26(\mathrm{~s}$, 2H), 3.79 (s, 3H), 2.34 (s, 3H). ${ }^{13} \mathrm{C}$ NMR (100 MHz, $\left.\mathrm{CDCl}_{3}\right) \delta 210.2,159.6,143.5,135.5,132.9$, 129.5, 128.6, 128.0, 127.3, 126.5, 114.5, 113.7, 99.7, 85.9, 79.8, 78.8, 55.3, 47.2, 36.6, 21.5. HRMS-ESI (m/z): $[\mathrm{M}+\mathrm{H}]^{+}$calcd for $\mathrm{C}_{27} \mathrm{H}_{26} \mathrm{NO}_{3} \mathrm{~S}, 444.1633$; found: 444.1615 . 


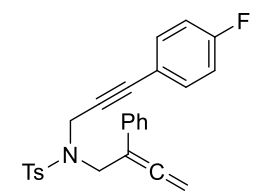

N-(3-(4-fluorophenyl)prop-2-yn-1-yl)-4-methyl-N-(2-phenylbuta-2,3-dien-1-yl)benzenesulfon amide (3d)

Compound 3d was prepared according to the general procedure from (3-bromoprop-1-yn-1-yl)benzene (390 mg, $2 \mathrm{mmol}$ ) and was isolated as a yellow oil (260 mg, 30\% total yield) after short column chromatography (5\%-10\% EtOAc/Petroleum). ${ }^{1} \mathrm{H}$ NMR (400 MHz, $\left.\mathrm{CDCl}_{3}\right) \delta 7.80(\mathrm{~d}, J=8.3 \mathrm{~Hz}, 2 \mathrm{H}), 7.58(\mathrm{~d}, J=7.5 \mathrm{~Hz}, 2 \mathrm{H}), 7.35(\mathrm{t}, J=7.7 \mathrm{~Hz}, 2 \mathrm{H}), 7.24(\mathrm{t}, J=$ $8.6 \mathrm{~Hz}, 3 \mathrm{H}), 7.06-6.95(\mathrm{~m}, 2 \mathrm{H}), 6.96-6.87(\mathrm{~m}, 2 \mathrm{H}), 5.13(\mathrm{~s}, 2 \mathrm{H}), 4.34(\mathrm{t}, J=1.8 \mathrm{~Hz}, 2 \mathrm{H}), 4.25$ (s, 2H), $2.32(\mathrm{~s}, 3 \mathrm{H}) .{ }^{13} \mathrm{C}$ NMR $\left(100 \mathrm{MHz}, \mathrm{CDCl}_{3}\right) \delta 210.3,163.7,161.2,143.7,135.4,133.4$, 133.4, 133.2, 129.6, 128.6, 128.1, 127.4, 126.5, 118.4, 118.4, 115.5, 115.3, 99.7, 85.0, 81.1, 78.8, 47.3, 36.5, 21.5. HRMS-ESI (m/z): $[\mathrm{M}+\mathrm{H}]^{+}$calcd for $\mathrm{C}_{26} \mathrm{H}_{23} \mathrm{FNO}_{2} \mathrm{~S}$, 432.1434; found: 432.1448.

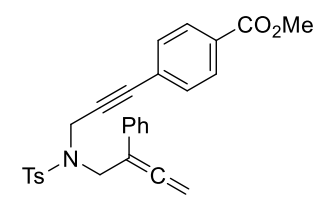

methyl 4-(3-((4-methyl-N-(2-phenylbuta-2,3-dien-1-yl)phenyl)sulfonamido)prop-1-yn-1-yl) benzoate (3e)

Compound $\mathbf{3 e}$ was prepared according to the general procedure from (3-bromoprop-1-yn-1-yl)benzene (390 mg, $2 \mathrm{mmol}$ ) and was isolated as a yellow oil (330 $\mathrm{mg}, 35 \%$ total yield) after short column chromatography (5\%-10\% EtOAc/Petroleum). ${ }^{1} \mathrm{H}$ NMR (400 MHz, $\left.\mathrm{CDCl}_{3}\right) \delta 7.90(\mathrm{~d}, J=8.3 \mathrm{~Hz}, 2 \mathrm{H}), 7.80(\mathrm{~d}, J=8.2 \mathrm{~Hz}, 2 \mathrm{H}), 7.58(\mathrm{~d}, J=7.7 \mathrm{~Hz}, 2 \mathrm{H}), 7.35(\mathrm{t}, J=$ $7.7 \mathrm{~Hz}, 2 \mathrm{H}), 7.25(\mathrm{~d}, J=8.1 \mathrm{~Hz}, 3 \mathrm{H}), 7.07(\mathrm{~d}, J=8.3 \mathrm{~Hz}, 2 \mathrm{H}), 5.13(\mathrm{~s}, 2 \mathrm{H}), 4.35(\mathrm{~s}, 2 \mathrm{H}), 4.29(\mathrm{~s}$, 2H), $3.91(\mathrm{~s}, 3 \mathrm{H}), 2.31(\mathrm{~s}, 3 \mathrm{H}) .{ }^{13} \mathrm{C} \mathrm{NMR}\left(100 \mathrm{MHz}, \mathrm{CDCl}_{3}\right) \delta 210.2,166.4,143.8,135.3,133.1$, $131.4,129.6,129.2,128.6,128.0,127.4,126.9,126.5,99.6,85.3,84.5,78.8,52.3,47.4,36.5,21.5$. HRMS-ESI (m/z): $[\mathrm{M}+\mathrm{H}]^{+}$calcd for $\mathrm{C}_{28} \mathrm{H}_{26} \mathrm{NO}_{4} \mathrm{~S}, 472.1583$; found: 472.1577.

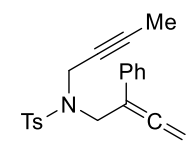

\section{N-(but-2-yn-1-yl)-4-methyl-N-(2-phenylbuta-2,3-dien-1-yl)benzenesulfonamide 3f}

Compound $\mathbf{3 f}$ was prepared according to the general procedure from 
(3-bromoprop-1-yn-1-yl)benzene (390 mg, $2 \mathrm{mmol}$ ) and was isolated as a yellow oil (210 mg, 30\% total yield) after short column chromatography (5\%-10\% EtOAc/Petroleum). ${ }^{1} \mathrm{H}$ NMR (400 MHz, $\left.\mathrm{CDCl}_{3}\right) \delta 7.77(\mathrm{~d}, J=8.1 \mathrm{~Hz}, 2 \mathrm{H}), 7.54(\mathrm{~d}, J=7.7 \mathrm{~Hz}, 2 \mathrm{H}), 7.32(\mathrm{dd}, J=14.1,7.6 \mathrm{~Hz}, 4 \mathrm{H}), 7.23$ (dd, $J=13.3,6.0 \mathrm{~Hz}, 1 \mathrm{H}), 5.11(\mathrm{~s}, 2 \mathrm{H}), 4.26(\mathrm{~s}, 2 \mathrm{H}), 4.00(\mathrm{~d}, J=2.0 \mathrm{~Hz}, 2 \mathrm{H}), 2.43$ (s, 3H), 1.49 (s, 3H). ${ }^{13} \mathrm{C} \mathrm{NMR}\left(100 \mathrm{MHz}, \mathrm{CDCl}_{3}\right) \delta 210.0,143.3,135.7,133.4,129.2,128.6,128.1,127.3,126.5$, $99.9, \quad 81.9, \quad 78.7, \quad 71.2, \quad 46.9, \quad 36.2, \quad 21.5, \quad 3.2$. Spectral data was consistent with N-(but-2-yn-1-yl)-4-methyl-N-(2-phenylbuta-2,3-dien-1-yl)benzenesulfonamide. ${ }^{1}$

\section{Typical Procedure for Borylative Cyclization}

To a solution of 1,6-allenynes $\left(0.1 \mathrm{mmol}, 1.0\right.$ equiv) in dichloromethane $(1 \mathrm{~mL})$ was added $\mathrm{BCl}_{3}$ ( $0.15 \mathrm{~mL}, 1 \mathrm{M}$ in $\mathrm{CH}_{2} \mathrm{Cl}_{2}, 1.5$ equiv) at room temperature. After 3 hours' stirring, a mixture of pinacol ( $0.11 \mathrm{mmol}, 1.1$ equiv) and $\mathrm{Et}_{3} \mathrm{~N}(1.5 \mathrm{mmol}, 15.0$ equiv) was added. The resulting mixture was stirred at room temperature for $30 \mathrm{~min}$ as monitored by TLC. Upon completion, the reaction mixture was quenched with water $(2 \mathrm{~mL})$ and extracted with $\mathrm{CH}_{2} \mathrm{Cl}_{2}(5 \mathrm{~mL} \times 3)$. The combined organic layers were dried over anhydrous $\mathrm{Na}_{2} \mathrm{SO}_{4}$, filtered and concentrated. The residue was purified by a short column chromatography on silica gel to afford borylative cyclization products.

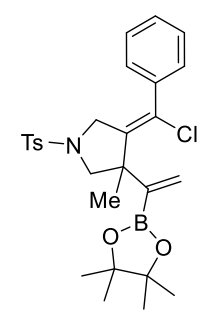

(E)-4-(chloro(phenyl)methylene)-3-methyl-3-(1-(4,4,5,5-tetramethyl-1,3,2-dioxaborolan-2-yl) vinyl)-1-tosylpyrrolidine (2a)

Prepared according to general procedure to give $\mathbf{2 a}(46 \mathrm{mg}, 90 \%)$ as a white solid after short column chromatography (5\%-10\% EtOAc/Petroleum). ${ }^{1} \mathrm{H}$ NMR $\left(400 \mathrm{MHz}, \mathrm{CDCl}_{3}\right) \delta 7.65(\mathrm{~d}, J=$ $7.5 \mathrm{~Hz}, 2 \mathrm{H}), 7.33$ (t, $J=9.3 \mathrm{~Hz}, 5 \mathrm{H}), 7.24(\mathrm{~d}, J=7.2 \mathrm{~Hz}, 2 \mathrm{H}), 6.02(\mathrm{~s}, 1 \mathrm{H}), 5.85(\mathrm{~s}, 1 \mathrm{H}), 3.97$ (d, $J$ $=13.3 \mathrm{~Hz}, 1 \mathrm{H}), 3.67(\mathrm{~d}, J=13.3 \mathrm{~Hz}, 1 \mathrm{H}), 3.15(\mathrm{~d}, J=8.8 \mathrm{~Hz}, 1 \mathrm{H}), 2.95(\mathrm{~d}, J=8.8 \mathrm{~Hz}, 1 \mathrm{H}), 2.43$ (s, 3H), 1.64 (s, 3H), 1.11 (s, 6H), 0.97 (s, 6H). $\left.{ }^{13} \mathrm{C} \mathrm{NMR} \mathrm{(100} \mathrm{MHz,} \mathrm{CDCl}_{3}\right) \delta$ 143.7, 140.9, 139.4, $131.2,129.5,129.4,128.5,128.4,127.7,123.5,83.3,61.3,53.9,50.6,24.7,24.3,21.5,20.6 .{ }^{11} \mathrm{~B}$ NMR (128 MHz, $\left.\mathrm{CDCl}_{3}\right) \delta$ 29.6. HRMS-ESI (m/z): $[\mathrm{M}+\mathrm{H}]^{+}$calcd for $\mathrm{C}_{27} \mathrm{H}_{34} \mathrm{BCINO}_{4} \mathrm{~S}$, 514.1990; found: 514.1996. 


\section{1 mmol scale synthesis of $2 a$}

To

a

solution

of

4-methyl-N-(2-methylbuta-2,3-dien-1-yl)-N-(3-phenylprop-2-yn-1-yl)benzenesulfonamide

$1 a$

(350 mg, $1.0 \mathrm{mmol}, 1.0$ equiv) in dichloromethane $(10 \mathrm{~mL})$ was added $\mathrm{BCl}_{3}(1.5 \mathrm{~mL}, 1 \mathrm{M}$ in

$\mathrm{CH}_{2} \mathrm{Cl}_{2}, 1.5$ equiv) at room temperature. After 3 hours' stirring, a mixture of pinacol (130 mg, 1.1 mmol, 1.1 equiv) and $\mathrm{Et}_{3} \mathrm{~N}(2.1 \mathrm{~mL}, 15.1 \mathrm{mmol}, 15.0$ equiv) was added. The resulting mixture was stirred at room temperature for $30 \mathrm{~min}$ as monitored by TLC. Upon completion, the reaction mixture was quenched with water $(20 \mathrm{~mL})$ and extracted with $\mathrm{CH}_{2} \mathrm{Cl}_{2}(25 \mathrm{~mL} \times 3)$. The combined organic layers were dried over anhydrous $\mathrm{Na}_{2} \mathrm{SO}_{4}$, filtered and concentrated. The residue was purified by a short column chromatography on silica gel to afford borylative cyclization product 2a (440 $\mathrm{mg}, 86 \%)$ as a white solid after short column chromatography (5\%-10\% EtOAc/Petroleum).

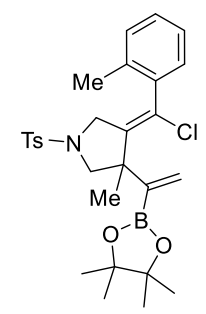

(E)-4-(chloro(o-tolyl)methylene)-3-methyl-3-(1-(4,4,5,5-tetramethyl-1,3,2-dioxaborolan-2-yl) vinyl)-1-tosylpyrrolidine (2b)

Prepared according to general procedure to give $\mathbf{2 b}(42 \mathrm{mg}, 80 \%)$ as a white solid after short column chromatography (5\%-10\% EtOAc/Petroleum). ${ }^{1} \mathrm{H}$ NMR $\left(400 \mathrm{MHz}, \mathrm{CDCl}_{3}\right) \delta 7.60(\mathrm{~d}, J=$ $7.5 \mathrm{~Hz}, 2 \mathrm{H}), 7.32(\mathrm{~d}, J=6.9 \mathrm{~Hz}, 2 \mathrm{H}), 7.26-7.20(\mathrm{~m}, 2 \mathrm{H}), 7.16-7.09(\mathrm{~m}, 1 \mathrm{H}), 7.01(\mathrm{~d}, J=7.0$ Hz, 1H), 6.04 (s, 1H), $5.87(\mathrm{~s}, 1 \mathrm{H}), 3.64(\mathrm{~d}, J=13.5 \mathrm{~Hz}, 1 \mathrm{H}), 3.45(\mathrm{~d}, J=13.6 \mathrm{~Hz}, 1 \mathrm{H}), 3.16(\mathrm{~d}, J$ $=8.5 \mathrm{~Hz}, 1 \mathrm{H}), 2.93(\mathrm{~d}, J=8.6 \mathrm{~Hz}, 1 \mathrm{H}), 2.42(\mathrm{~s}, 3 \mathrm{H}), 2.30(\mathrm{~s}, 3 \mathrm{H}), 1.63(\mathrm{~s}, 3 \mathrm{H}), 1.15(\mathrm{~s}, 6 \mathrm{H}), 0.98$ $(\mathrm{s}, 6 \mathrm{H}) .{ }^{13} \mathrm{C} \mathrm{NMR}\left(100 \mathrm{MHz}, \mathrm{CDCl}_{3}\right) \delta 143.7,141.4,138.6,136.1,131.3,131.0,129.6,129.1$, $128.4,127.5,126.1,122.9,83.5,61.5,53.7,50.1,25.0,24.2,21.6,20.8,19.0 .{ }^{11} \mathrm{~B}$ NMR (128 MHz, $\left.\mathrm{CDCl}_{3}\right) \delta$ 29.5. HRMS-ESI (m/z): $[\mathrm{M}+\mathrm{H}]^{+}$calcd for $\mathrm{C}_{28} \mathrm{H}_{36} \mathrm{BClNO}_{4} \mathrm{~S}$, 528.2147; found: 528.2132. 


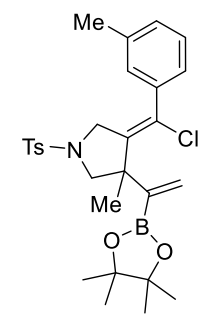

(E)-4-(chloro(m-tolyl)methylene)-3-methyl-3-(1-(4,4,5,5-tetramethyl-1,3,2-dioxaborolan-2-yl) vinyl)-1-tosylpyrrolidine (2c)

Prepared according to general procedure to give $2 \mathrm{c}(45 \mathrm{mg}, 85 \%)$ as a white solid after short column chromatography (5\%-10\% EtOAc/Petroleum). ${ }^{1} \mathrm{H}$ NMR $\left(400 \mathrm{MHz}, \mathrm{CDCl}_{3}\right) \delta 7.56(\mathrm{~d}, J=$ $7.7 \mathrm{~Hz}, 2 \mathrm{H}), 7.26(\mathrm{~d}, J=7.5 \mathrm{~Hz}, 2 \mathrm{H}), 7.14(\mathrm{t}, J=7.3 \mathrm{~Hz}, 1 \mathrm{H}), 7.06-6.98(\mathrm{~m}, 2 \mathrm{H}), 6.93(\mathrm{~d}, J=$ $7.5 \mathrm{~Hz}, 1 \mathrm{H}), 5.94(\mathrm{~s}, 1 \mathrm{H}), 5.77(\mathrm{~s}, 1 \mathrm{H}), 3.88(\mathrm{~d}, J=13.3 \mathrm{~Hz}, 1 \mathrm{H}), 3.59$ (d, $J=13.2 \mathrm{~Hz}, 1 \mathrm{H}), 3.06$ $(\mathrm{d}, J=8.8 \mathrm{~Hz}, 1 \mathrm{H}), 2.86(\mathrm{~d}, J=8.7 \mathrm{~Hz}, 1 \mathrm{H}), 2.35(\mathrm{~s}, 3 \mathrm{H}), 2.26(\mathrm{~s}, 3 \mathrm{H}), 1.55(\mathrm{~s}, 3 \mathrm{H}), 1.03(\mathrm{~s}, 6 \mathrm{H})$, $0.88(\mathrm{~s}, 6 \mathrm{H}) .{ }^{13} \mathrm{C} \mathrm{NMR}\left(100 \mathrm{MHz}, \mathrm{CDCl}_{3}\right) \delta 143.8,140.8,139.5,138.3,131.2,129.6,129.5,129.4$, 128.6, 128.4, 124.7, 123.7, 83.4, 61.4, 54.1, 50.5, 24.8, 24.3, 21.6, 21.4, 20.7. ${ }^{11}$ B NMR (128 MHz, $\left.\mathrm{CDCl}_{3}\right) \delta$ 29.4. HRMS-ESI (m/z): $[\mathrm{M}+\mathrm{H}]^{+}$calcd for $\mathrm{C}_{28} \mathrm{H}_{36} \mathrm{BClNO}_{4} \mathrm{~S}, 528.2147$; found: 528.2148.

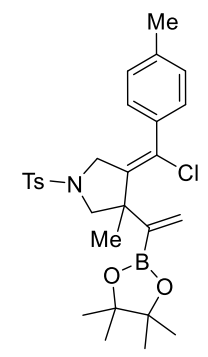

(E)-4-(chloro(p-tolyl)methylene)-3-methyl-3-(1-(4,4,5,5-tetramethyl-1,3,2-dioxaborolan-2-yl) vinyl)-1-tosylpyrrolidine (2d)

Prepared according to general procedure to give $\mathbf{2 d}(46 \mathrm{mg}, 87 \%)$ as a white solid after short column chromatography (5\%-10\% EtOAc/Petroleum). ${ }^{1} \mathrm{H}$ NMR $\left(400 \mathrm{MHz}, \mathrm{CDCl}_{3}\right) \delta 7.65(\mathrm{~d}, J=$ $8.2 \mathrm{~Hz}, 2 \mathrm{H}), 7.34(\mathrm{~d}, J=8.0 \mathrm{~Hz}, 2 \mathrm{H}), 7.15(\mathrm{~d}, J=8.7 \mathrm{~Hz}, 2 \mathrm{H}), 7.13(\mathrm{~d}, J=8.7 \mathrm{~Hz}, 2 \mathrm{H}), 6.01(\mathrm{~d}, J$ $=2.1 \mathrm{~Hz}, 1 \mathrm{H}), 5.84(\mathrm{~d}, J=1.9 \mathrm{~Hz}, 1 \mathrm{H}), 3.97(\mathrm{~d}, J=13.3 \mathrm{~Hz}, 1 \mathrm{H}), 3.67(\mathrm{~d}, J=13.3 \mathrm{~Hz}, 1 \mathrm{H}), 3.14$ $(\mathrm{d}, J=8.8 \mathrm{~Hz}, 1 \mathrm{H}), 2.94(\mathrm{~d}, J=8.8 \mathrm{~Hz}, 1 \mathrm{H}), 2.43(\mathrm{~s}, 3 \mathrm{H}), 2.36(\mathrm{~s}, 3 \mathrm{H}), 1.63(\mathrm{~s}, 3 \mathrm{H}), 1.10(\mathrm{~s}, 6 \mathrm{H})$, $0.96(\mathrm{~s}, 6 \mathrm{H}) .{ }^{13} \mathrm{C} \mathrm{NMR}\left(100 \mathrm{MHz}, \mathrm{CDCl}_{3}\right) \delta 143.7,140.6,138.6,136.8,131.3,129.6,129.4,129.2$, $128.5,127.7,123.8,83.4,61.5,54.1,50.6,24.8,24.4,21.6,21.4,20.8 .{ }^{11} \mathrm{~B}$ NMR (128 MHz, $\left.\mathrm{CDCl}_{3}\right) \delta$ 29.7. HRMS-ESI (m/z): $[\mathrm{M}+\mathrm{H}]^{+}$calcd for $\mathrm{C}_{28} \mathrm{H}_{36} \mathrm{BClNO}_{4} \mathrm{~S}, 528.2147$; found: 528.2105. 


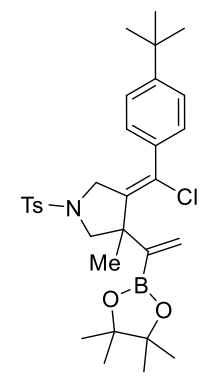

(E)-4-((4-(tert-butyl)phenyl)chloromethylene)-3-methyl-3-(1-(4,4,5,5-tetramethyl-1,3,2-dioxa borolan-2-yl)vinyl)-1-tosylpyrrolidine (2e)

Prepared according to general procedure to give $\mathbf{2 e}(47 \mathrm{mg}, 82 \%)$ as a white solid after short column chromatography (5\%-10\% EtOAc/Petroleum). ${ }^{1} \mathrm{H}$ NMR $\left(400 \mathrm{MHz}, \mathrm{CDCl}_{3}\right) \delta 7.67(\mathrm{~d}, J=$ $8.2 \mathrm{~Hz}, 2 \mathrm{H}), 7.36(\mathrm{~d}, J=3.4 \mathrm{~Hz}, 2 \mathrm{H}), 7.34(\mathrm{~d}, J=4.0 \mathrm{~Hz}, 2 \mathrm{H}), 7.16(\mathrm{~d}, J=8.5 \mathrm{~Hz}, 2 \mathrm{H}), 6.01(\mathrm{~d}, J$ $=2.2 \mathrm{~Hz}, 1 \mathrm{H}), 5.84(\mathrm{~d}, J=2.2 \mathrm{~Hz}, 1 \mathrm{H}), 4.00(\mathrm{~d}, J=13.3 \mathrm{~Hz}, 1 \mathrm{H}), 3.71(\mathrm{~d}, J=13.3 \mathrm{~Hz}, 1 \mathrm{H}), 3.13$ $(\mathrm{d}, J=8.8 \mathrm{~Hz}, 1 \mathrm{H}), 2.94(\mathrm{~d}, J=8.8 \mathrm{~Hz}, 1 \mathrm{H}), 2.44(\mathrm{~s}, 3 \mathrm{H}), 1.63(\mathrm{~s}, 3 \mathrm{H}), 1.34(\mathrm{~s}, 9 \mathrm{H}), 1.11(\mathrm{~s}, 6 \mathrm{H})$, $0.96(\mathrm{~s}, 6 \mathrm{H}) .{ }^{13} \mathrm{C} \mathrm{NMR}\left(100 \mathrm{MHz}, \mathrm{CDCl}_{3}\right) \delta 151.5,143.6,140.5,136.5,131.2,129.5,129.3,128.4$, $127.4,125.4,123.7,83.3,61.4,54.1,50.5,34.7,31.3,24.7,24.3,21.5,20.7 .{ }^{11} \mathrm{~B}$ NMR $(128 \mathrm{MHz}$ $\left.\mathrm{CDCl}_{3}\right) \delta$ 29.7. HRMS-ESI (m/z): $[\mathrm{M}+\mathrm{H}]^{+}$calcd for $\mathrm{C}_{31} \mathrm{H}_{42} \mathrm{BCINO}_{4} \mathrm{~S}$, 570.2616; found: 570.2612.<smiles>[Y5]N1CC(=C(Cl)c2ccc(OC)cc2)C([M])(C(=C)B2OC(C)(C)C(C)(C)O2)C1</smiles>

(E)-4-(chloro(4-methoxyphenyl)methylene)-3-methyl-3-(1-(4,4,5,5-tetramethyl-1,3,2-dioxabo rolan-2-yl)vinyl)-1-tosylpyrrolidine (2f)

Prepared according to general procedure to give $\mathbf{2 f}(14 \mathrm{mg}, 26 \%)$ as a white solid after short column chromatography (5\%-10\% EtOAc/Petroleum). ${ }^{1} \mathrm{H}$ NMR $\left(400 \mathrm{MHz}, \mathrm{CDCl}_{3}\right) \delta 7.65(\mathrm{~d}, J=$ $8.2 \mathrm{~Hz}, 2 \mathrm{H}), 7.35(\mathrm{~d}, J=8.0 \mathrm{~Hz}, 2 \mathrm{H}), 7.18(\mathrm{~d}, J=8.8 \mathrm{~Hz}, 2 \mathrm{H}), 6.86(\mathrm{~d}, J=8.8 \mathrm{~Hz}, 2 \mathrm{H}), 6.00(\mathrm{~d}, J$ $=2.2 \mathrm{~Hz}, 1 \mathrm{H}), 5.84(\mathrm{~d}, J=2.1 \mathrm{~Hz}, 1 \mathrm{H}), 3.97(\mathrm{~d}, J=13.2 \mathrm{~Hz}, 1 \mathrm{H}), 3.83(\mathrm{~s}, 3 \mathrm{H}), 3.66(\mathrm{~d}, J=13.3$ $\mathrm{Hz}, 1 \mathrm{H}), 3.14(\mathrm{~d}, J=8.8 \mathrm{~Hz}, 1 \mathrm{H}), 2.94(\mathrm{~d}, J=8.9 \mathrm{~Hz}, 1 \mathrm{H}), 2.44(\mathrm{~s}, 3 \mathrm{H}), 1.62(\mathrm{~s}, 3 \mathrm{H}), 1.10(\mathrm{~s}, 6 \mathrm{H})$, 0.96 (s, 6H). ${ }^{13} \mathrm{C}$ NMR (100 MHz, $\left.\mathrm{CDCl}_{3}\right) \delta 159.5,143.6,140.3,131.9,131.2,129.5,129.3,129.1$, $128.4,123.5,113.8,83.2,61.4,55.4,54.0,50.5,24.7,24.3,21.5,20.7 .{ }^{11} \mathrm{~B}$ NMR (128 MHz, $\left.\mathrm{CDCl}_{3}\right) \delta$ 29.3. HRMS-ESI (m/z): $[\mathrm{M}+\mathrm{H}]^{+}$calcd for $\mathrm{C}_{28} \mathrm{H}_{36} \mathrm{BCINO}_{5} \mathrm{~S}$, 544.2096; found: 544.2086. 


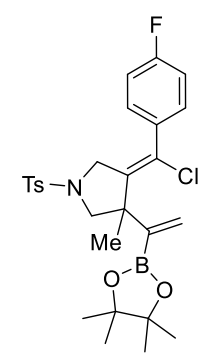

(E)-4-(chloro(4-fluorophenyl)methylene)-3-methyl-3-(1-(4,4,5,5-tetramethyl-1,3,2-dioxaborol an-2-yl)vinyl)-1-tosylpyrrolidine (2g)

Prepared according to general procedure to give $\mathbf{2 g}(45 \mathrm{mg}, 85 \%)$ as a white solid after short column chromatography (5\%-10\% EtOAc/Petroleum). ${ }^{1} \mathrm{H}$ NMR $\left(400 \mathrm{MHz}, \mathrm{CDCl}_{3}\right) \delta 7.65(\mathrm{~d}, J=$ $8.2 \mathrm{~Hz}, 2 \mathrm{H}), 7.35(\mathrm{~d}, J=8.0 \mathrm{~Hz}, 2 \mathrm{H}), 7.25-7.18(\mathrm{~m}, 2 \mathrm{H}), 7.08-6.98(\mathrm{~m}, 2 \mathrm{H}), 6.03(\mathrm{~d}, J=2.1$ $\mathrm{Hz}, 1 \mathrm{H}), 5.85(\mathrm{~d}, J=2.1 \mathrm{~Hz}, 1 \mathrm{H}), 3.94(\mathrm{~d}, J=13.3 \mathrm{~Hz}, 1 \mathrm{H}), 3.63(\mathrm{~d}, J=13.3 \mathrm{~Hz}, 1 \mathrm{H}), 3.15(\mathrm{~d}, J=$ $8.8 \mathrm{~Hz}, 1 \mathrm{H}), 2.92(\mathrm{~d}, J=8.9 \mathrm{~Hz}, 1 \mathrm{H}), 2.44(\mathrm{~s}, 3 \mathrm{H}), 1.63(\mathrm{~s}, 3 \mathrm{H}), 1.10(\mathrm{~s}, 6 \mathrm{H}), 0.96(\mathrm{~s}, 6 \mathrm{H}) .{ }^{13} \mathrm{C}$ NMR $\left(100 \mathrm{MHz}, \mathrm{CDCl}_{3}\right) \delta 163.6,161.1,143.7,141.4,135.5,135.5,131.0,129.7,129.7,129.6$, $129.6,128.4,122.5,115.7,115.4,83.3,61.3,53.9,50.5,24.7,24.2,21.5,20.6 .{ }^{11} \mathrm{~B}$ NMR $(128$ $\left.\mathrm{MHz}, \mathrm{CDCl}_{3}\right) \delta$ 29.5. HRMS-ESI (m/z): $[\mathrm{M}+\mathrm{H}]^{+}$calcd for $\mathrm{C}_{27} \mathrm{H}_{33} \mathrm{BClFNO}_{4} \mathrm{~S}, 532.1896$; found: 532.1828 .

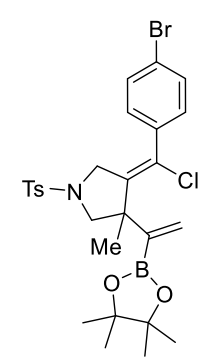

(E)-4-((4-bromophenyl)chloromethylene)-3-methyl-3-(1-(4,4,5,5-tetramethyl-1,3,2-dioxaboro lan-2-yl)vinyl)-1-tosylpyrrolidine (2h)

Prepared according to general procedure to give $\mathbf{2 h}(50 \mathrm{mg}, 84 \%)$ as a white solid after short column chromatography $\left(5 \%-10 \%\right.$ EtOAc/Petroleum). ${ }^{1} \mathrm{H}$ NMR $\left(400 \mathrm{MHz}, \mathrm{CDCl}_{3}\right) \delta 7.65(\mathrm{~d}, J=$ $8.2 \mathrm{~Hz}, 2 \mathrm{H}), 7.49(\mathrm{~d}, J=8.4 \mathrm{~Hz}, 2 \mathrm{H}), 7.35(\mathrm{~d}, J=8.1 \mathrm{~Hz}, 2 \mathrm{H}), 7.12(\mathrm{~d}, J=8.4 \mathrm{~Hz}, 2 \mathrm{H}), 6.02(\mathrm{~d}, J$ $=2.0 \mathrm{~Hz}, 1 \mathrm{H}), 5.85(\mathrm{~d}, J=1.9 \mathrm{~Hz}, 1 \mathrm{H}), 3.94(\mathrm{~d}, J=13.3 \mathrm{~Hz}, 1 \mathrm{H}), 3.62(\mathrm{~d}, J=13.3 \mathrm{~Hz}, 1 \mathrm{H}), 3.15$ $(\mathrm{d}, J=8.8 \mathrm{~Hz}, 1 \mathrm{H}), 2.91(\mathrm{~d}, J=8.9 \mathrm{~Hz}, 1 \mathrm{H}), 2.44(\mathrm{~s}, 3 \mathrm{H}), 1.62(\mathrm{~s}, 3 \mathrm{H}), 1.09(\mathrm{~s}, 6 \mathrm{H}), 0.95(\mathrm{~s}, 6 \mathrm{H})$. ${ }^{13} \mathrm{C}$ NMR $\left(100 \mathrm{MHz}, \mathrm{CDCl}_{3}\right) \delta 143.8,141.8,138.2,131.7,131.0,129.7,129.6,129.4,128.4$, 122.6, 122.3, 83.3, 61.3, 53.9, 50.6, 24.7, 24.2, 21.5, 20.5. ${ }^{11} \mathrm{~B}$ NMR $\left(128 \mathrm{MHz}, \mathrm{CDCl}_{3}\right) \delta 29.2$. 
HRMS-ESI (m/z): $[\mathrm{M}+\mathrm{H}]^{+}$calcd for $\mathrm{C}_{27} \mathrm{H}_{33} \mathrm{BBrClNO}_{4} \mathrm{~S}, 592.1095$; found: 592.1096 .

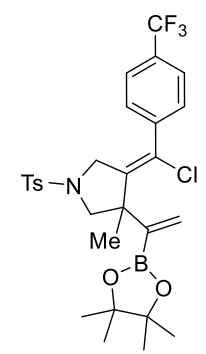

(E)-4-(chloro(4-(trifluoromethyl)phenyl)methylene)-3-methyl-3-(1-(4,4,5,5-tetramethyl-1,3,2dioxaborolan-2-yl)vinyl)-1-tosylpyrrolidine (2i)

Prepared according to general procedure to give $2 \mathbf{i}$ (52 $\mathrm{mg}, 89 \%$ ) as a white solid after short column chromatography (5\%-10\% EtOAc/Petroleum). ${ }^{1} \mathrm{H}$ NMR $\left(400 \mathrm{MHz}, \mathrm{CDCl}_{3}\right) \delta 7.65(\mathrm{~d}, J=$ $8.3 \mathrm{~Hz}, 2 \mathrm{H}), 7.63(\mathrm{~d}, J=8.3 \mathrm{~Hz}, 2 \mathrm{H}), 7.37(\mathrm{~d}, J=4.6 \mathrm{~Hz}, 2 \mathrm{H}), 7.35(\mathrm{~d}, J=4.5 \mathrm{~Hz}, 2 \mathrm{H}), 6.05(\mathrm{~d}, J$ $=2.1 \mathrm{~Hz}, 1 \mathrm{H}), 5.87(\mathrm{~d}, J=2.0 \mathrm{~Hz}, 1 \mathrm{H}), 3.94(\mathrm{~d}, J=13.3 \mathrm{~Hz}, 1 \mathrm{H}), 3.66(\mathrm{~d}, J=13.3 \mathrm{~Hz}, 1 \mathrm{H}), 3.17$ $(\mathrm{d}, J=8.9 \mathrm{~Hz}, 1 \mathrm{H}), 2.92(\mathrm{~d}, J=8.9 \mathrm{~Hz}, 1 \mathrm{H}), 2.44$ (s, 3H), $1.64(\mathrm{~s}, 3 \mathrm{H}), 1.11(\mathrm{~s}, 6 \mathrm{H}), 0.96(\mathrm{~s}, 6 \mathrm{H})$. ${ }^{13} \mathrm{C}$ NMR $\left(100 \mathrm{MHz}, \mathrm{CDCl}_{3}\right) \delta 143.8,142.8,142.7,131.0,130.7,130.4,129.9,129.6,128.4$, $128.2,125.7,125.6,125.6,125.5,125.2,122.5,121.9,83.4,61.3,53.9,50.8,24.7,24.3,21.5,20.5$. ${ }^{11} \mathrm{~B}$ NMR $\left(128 \mathrm{MHz}, \mathrm{CDCl}_{3}\right) \delta$ 29.5. HRMS-ESI $(\mathrm{m} / \mathrm{z}):[\mathrm{M}+\mathrm{H}]^{+}$calcd for $\mathrm{C}_{28} \mathrm{H}_{33} \mathrm{BClF}_{3} \mathrm{NO}_{4} \mathrm{~S}$, 582.1864; found: 582.1834 .

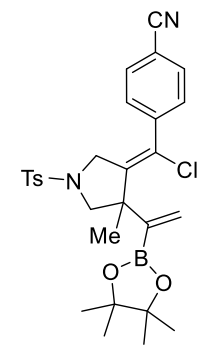

(E)-4-(chloro(4-methyl-4-(1-(4,4,5,5-tetramethyl-1,3,2-dioxaborolan-2-yl)vinyl)-1-tosylpyrrol idin-3-ylidene)methyl)benzonitrile (2j)

Prepared according to general procedure to give $\mathbf{2} \mathbf{j}$ (42 $\mathrm{mg}, 78 \%$ ) as a white solid after short column chromatography (5\%-10\% EtOAc/Petroleum). ${ }^{1} \mathrm{H}$ NMR (400 MHz, $\left.\mathrm{CDCl}_{3}\right) \delta 7.67(\mathrm{~d}, J=$ $6.1 \mathrm{~Hz}, 2 \mathrm{H}), 7.65(\mathrm{~d}, J=5.9 \mathrm{~Hz}, 2 \mathrm{H}), 7.36(\mathrm{~d}, J=8.2 \mathrm{~Hz}, 4 \mathrm{H}), 6.05(\mathrm{~d}, J=2.0 \mathrm{~Hz}, 1 \mathrm{H}), 5.87(\mathrm{~d}, J$ $=1.9 \mathrm{~Hz}, 1 \mathrm{H}), 3.94(\mathrm{~d}, J=13.3 \mathrm{~Hz}, 1 \mathrm{H}), 3.65(\mathrm{~d}, J=13.4 \mathrm{~Hz}, 1 \mathrm{H}), 3.17(\mathrm{~d}, J=8.9 \mathrm{~Hz}, 1 \mathrm{H}), 2.91$ (d, $J=8.9 \mathrm{~Hz}, 1 \mathrm{H}), 2.44$ (s, 3H), 1.63 (s, 3H), 1.10 (s, 6H), 0.96 (s, 6H). ${ }^{13} \mathrm{C}$ NMR (100 MHz, $\left.\mathrm{CDCl}_{3}\right) \delta 143.9,143.6,143.5,132.4,131.0,130.1,129.6,128.6,128.3,121.4,118.2,112.3,83.4$, 
61.2, 53.8, 50.9, 24.7, 24.3, 21.5, 20.4. ${ }^{11}$ B NMR (128 MHz, $\left.\mathrm{CDCl}_{3}\right) \delta$ 29.4. HRMS-ESI (m/z): $[\mathrm{M}+\mathrm{Na}]^{+}$calcd for $\mathrm{C}_{28} \mathrm{H}_{32} \mathrm{BClN}_{2} \mathrm{NaO}_{4} \mathrm{~S}, 561.1762$; found: 561.1741 .

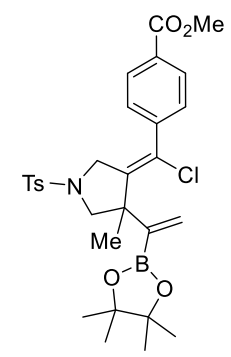

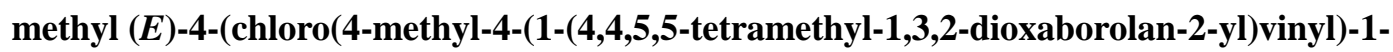
tosylpyrrolidin-3-ylidene)methyl)benzoate (2k)

Prepared according to general procedure to give $2 \mathbf{k}(50 \mathrm{mg}, 87 \%)$ as a white solid after short column chromatography (5\%-10\% EtOAc/Petroleum). ${ }^{1} \mathrm{H}$ NMR $\left(400 \mathrm{MHz}, \mathrm{CDCl}_{3}\right) \delta 8.03(\mathrm{~d}, J=$ $8.2 \mathrm{~Hz}, 2 \mathrm{H}), 7.64(\mathrm{~d}, J=8.1 \mathrm{~Hz}, 2 \mathrm{H}), 7.35(\mathrm{~d}, J=8.2 \mathrm{~Hz}, 2 \mathrm{H}), 7.32(\mathrm{~d}, J=8.4 \mathrm{~Hz}, 2 \mathrm{H}), 6.04(\mathrm{~d}, J$ $=1.8 \mathrm{~Hz}, 1 \mathrm{H}), 5.86(\mathrm{~d}, J=1.6 \mathrm{~Hz}, 1 \mathrm{H}), 4.03-3.87(\mathrm{~m}, 4 \mathrm{H}), 3.66(\mathrm{~d}, J=13.4 \mathrm{~Hz}, 1 \mathrm{H}), 3.16(\mathrm{~d}, J$ $=8.8 \mathrm{~Hz}, 1 \mathrm{H}), 2.92(\mathrm{~d}, J=8.9 \mathrm{~Hz}, 1 \mathrm{H}), 2.44(\mathrm{~s}, 3 \mathrm{H}), 1.64(\mathrm{~s}, 3 \mathrm{H}), 1.10(\mathrm{~s}, 6 \mathrm{H}), 0.96(\mathrm{~s}, 6 \mathrm{H}) .{ }^{13} \mathrm{C}$ NMR $\left(100 \mathrm{MHz}, \mathrm{CDCl}_{3}\right) \delta 166.6,143.9,143.8,142.5,131.1,130.2,129.9,129.9,129.7,128.5$, 128.0, 122.5, 83.5, 61.4, 54.0, 52.4, 50.9, 24.8, 24.4, 21.6, 20.6. ${ }^{11} \mathrm{~B}$ NMR $\left(128 \mathrm{MHz}, \mathrm{CDCl}_{3}\right) \delta$ 29.7. HRMS-ESI (m/z): $[\mathrm{M}+\mathrm{H}]^{+}$calcd for $\mathrm{C}_{29} \mathrm{H}_{36} \mathrm{BCINO}_{6} \mathrm{~S}, 572.2045$; found: 572.1990 .

4-(chloro(naphthalen-2-yl)methylene)-3-methyl-3-(1-(4,4,5,5-tetramethyl-1,3,2-dioxaborolan2-yl)vinyl)-1-tosylpyrrolidine (2l)

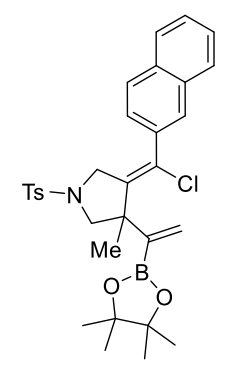

Prepared according to general procedure to give 21 (46 $\mathrm{mg}, 82 \%)$ as a white solid after short column chromatography (5\%-10\% EtOAc/Petroleum). The title compound was obtained as a mixture of distereoisomers (2:1). ${ }^{1} \mathrm{H}$ NMR $\left(400 \mathrm{MHz}, \mathrm{CDCl}_{3}\right) \delta 8.02-7.80(\mathrm{~m}, 3 \mathrm{H}), 7.61-7.45$ (m, 4H), $7.45-7.35(\mathrm{~m}, 1 \mathrm{H}), 7.30(\mathrm{~d}, J=7.4 \mathrm{~Hz}, 1 \mathrm{H}), 7.28-7.22(\mathrm{~m}, 2 \mathrm{H}), 6.12(\mathrm{~d}, J=24.9 \mathrm{~Hz}$, 1H), $5.97(\mathrm{~d}, J=22.7 \mathrm{~Hz}, 1 \mathrm{H}), 3.73(\mathrm{dd}, J=84.3,14.0 \mathrm{~Hz}, 1 \mathrm{H}), 3.47$ (dd, $J=46.4,13.6 \mathrm{~Hz}, 1 \mathrm{H})$, $3.19(\mathrm{t}, J=8.0 \mathrm{~Hz}, 1 \mathrm{H}), 3.02(\mathrm{dd}, J=18.6,8.9 \mathrm{~Hz}, 1 \mathrm{H}), 2.42(\mathrm{~s}, 3 \mathrm{H}), 1.75(\mathrm{~d}, J=22.2 \mathrm{~Hz}, 3 \mathrm{H})$, 
$1.20(\mathrm{~s}, 6 \mathrm{H}), 1.02(\mathrm{~s}, 6 \mathrm{H}) .{ }^{13} \mathrm{C} \mathrm{NMR}\left(100 \mathrm{MHz}, \mathrm{CDCl}_{3}\right) \delta 143.6,143.5,143.0,142.1,136.8,136.3$, 134.0, 133.8, 131.4, 131.3, 130.7, 130.0, 129.7, 129.5, 129.5, 129.2, 128.7, 128.5, 128.3, 127.3, $127.1,126.5,126.1,126.0,125.8,125.4,125.1,124.5,122.5,121.6,83.5,83.4,61.4,61.3,53.6$, 53.2, 50.8, 50.3, 25.1, 24.9, 24.2, 23.9, 21.5, 21.4, 20.8. ${ }^{11} \mathrm{~B}$ NMR $\left(128 \mathrm{MHz}, \mathrm{CDCl}_{3}\right) \delta 30.1$. HRMS-ESI (m/z): $[\mathrm{M}+\mathrm{H}]^{+}$calcd for $\mathrm{C}_{31} \mathrm{H}_{36} \mathrm{BCINO}_{4} \mathrm{~S}, 564.2147$; found: 564.2195.

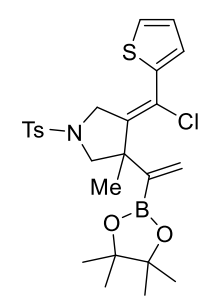

(E)-4-(chloro(thiophen-2-yl)methylene)-3-methyl-3-(1-(4,4,5,5-tetramethyl-1,3,2-dioxaborola n-2-yl)vinyl)-1-tosylpyrrolidine (2m)

Prepared according to general procedure to give $\mathbf{2 m}(42 \mathrm{mg}, 81 \%)$ as a white solid after short column chromatography (5\%-10\% EtOAc/Petroleum). ${ }^{1} \mathrm{H}$ NMR $\left(400 \mathrm{MHz}, \mathrm{CDCl}_{3}\right) \delta 7.71(\mathrm{~d}, J=$ $8.2 \mathrm{~Hz}, 2 \mathrm{H}), 7.30(\mathrm{~d}, J=8.2 \mathrm{~Hz}, 2 \mathrm{H}), 7.26-7.24(\mathrm{~m}, 1 \mathrm{H}), 7.07(\mathrm{~d}, J=3.5 \mathrm{~Hz}, 1 \mathrm{H}), 6.92(\mathrm{dd}, J=$ 5.0, $3.7 \mathrm{~Hz}, 1 \mathrm{H}), 5.80(\mathrm{~s}, 1 \mathrm{H}), 5.12(\mathrm{~s}, 1 \mathrm{H}), 4.15(\mathrm{~d}, J=14.9 \mathrm{~Hz}, 1 \mathrm{H}), 3.93(\mathrm{~d}, J=13.3 \mathrm{~Hz}, 1 \mathrm{H})$, $3.60(\mathrm{~d}, J=14.8 \mathrm{~Hz}, 1 \mathrm{H}), 3.43(\mathrm{~d}, J=13.3 \mathrm{~Hz}, 1 \mathrm{H}), 2.42(\mathrm{~s}, 3 \mathrm{H}), 1.90(\mathrm{~s}, 3 \mathrm{H}), 1.22(\mathrm{~s}, 6 \mathrm{H}), 1.20$ (s, 6H). ${ }^{13} \mathrm{C}$ NMR $\left(100 \mathrm{MHz}, \mathrm{CDCl}_{3}\right) \delta 152.1,146.9,145.5,143.3,136.3,129.7,128.1,127.2$, 126.6, $126.5,120.1,83.9,69.2,60.7,49.2,28.6,24.6,24.6,21.5 .{ }^{11} \mathrm{~B}$ NMR $\left(128 \mathrm{MHz}, \mathrm{CDCl}_{3}\right) \delta$ 30.2. HRMS-ESI (m/z): $[\mathrm{M}+\mathrm{H}]^{+}$calcd for $\mathrm{C}_{25} \mathrm{H}_{32} \mathrm{BCINO}_{4} \mathrm{~S}_{2}$, 520.1554; found: 520.1525 .

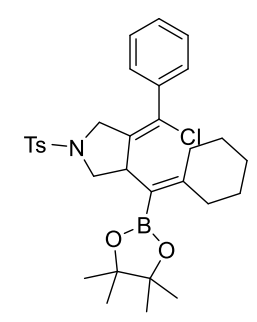

(E)-4-(chloro(phenyl)methylene)-3-(cyclohexylidene(4,4,5,5-tetramethyl-1,3,2-dioxaborolan-

\section{2-yl)methyl)-3-methyl-1-tosylpyrrolidine (2n)}

Prepared according to general procedure to give $\mathbf{2 n}(46 \mathrm{mg}, 81 \%)$ as a white solid after short column chromatography (5\%-10\% EtOAc/Petroleum). ${ }^{1} \mathrm{H}$ NMR $\left(400 \mathrm{MHz}, \mathrm{CDCl}_{3}\right) \delta 7.79(\mathrm{~d}, J=$ $8.2 \mathrm{~Hz}, 2 \mathrm{H}), 7.38(\mathrm{~d}, J=8.1 \mathrm{~Hz}, 2 \mathrm{H}), 7.23-7.16(\mathrm{~m}, 3 \mathrm{H}), 7.13(\mathrm{dd}, J=6.6,3.0 \mathrm{~Hz}, 2 \mathrm{H}), 4.40(\mathrm{~d}$, $J=14.6 \mathrm{~Hz}, 1 \mathrm{H}), 4.00(\mathrm{dd}, J=12.6,5.5 \mathrm{~Hz}, 1 \mathrm{H}), 3.75(\mathrm{dd}, J=14.6,2.4 \mathrm{~Hz}, 1 \mathrm{H}), 3.65(\mathrm{t}, J=9.1$ $\mathrm{Hz}, 1 \mathrm{H}), 3.04(\mathrm{t}, J=9.4 \mathrm{~Hz}, 1 \mathrm{H}), 2.45(\mathrm{~s}, 3 \mathrm{H}), 2.14-2.06(\mathrm{~m}, 1 \mathrm{H}), 2.03-1.94(\mathrm{~m}, 1 \mathrm{H}), 1.90-$ 
$1.76(\mathrm{~m}, 2 \mathrm{H}), 1.38-1.22(\mathrm{~m}, 6 \mathrm{H}), 1.01(\mathrm{~s}, 6 \mathrm{H}), 0.97(\mathrm{~s}, 6 \mathrm{H}) .{ }^{13} \mathrm{C} \mathrm{NMR}\left(100 \mathrm{MHz}, \mathrm{CDCl}_{3}\right) \delta$ 156.3, 143.6, 140.2, 137.1, 132.1, 129.6, 128.9, 128.4, 128.1, 127.6, 124.1, 82.5, 55.0, 54.6, 43.1, 34.4, 30.9, 28.1, 27.8, 26.6, 25.0, 24.3, 21.5. ${ }^{11} \mathrm{~B}$ NMR (128 MHz, $\left.\mathrm{CDCl}_{3}\right) \delta$ 29.5. HRMS-ESI (m/z): $[\mathrm{M}+\mathrm{H}]^{+}$calcd for $\mathrm{C}_{31} \mathrm{H}_{40} \mathrm{BCINO} \mathrm{S}_{4} \mathrm{~S}, 568.2460$; found: 568.2416 .

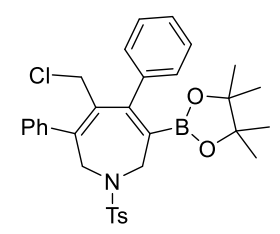

4-(chloromethyl)-3,5-diphenyl-6-(4,4,5,5-tetramethyl-1,3,2-dioxaborolan-2-yl)-1-tosyl-2,7-dih ydro-1H-azepine (4a)

Prepared according to general procedure to give $4 \mathbf{a}(50 \mathrm{mg}, 87 \%)$ as a white solid after short column chromatography (5\%-10\% EtOAc/Petroleum). ${ }^{1} \mathrm{H}$ NMR $\left(400 \mathrm{MHz}, \mathrm{CDCl}_{3}\right) \delta 7.37$ (d, J= $7.3 \mathrm{~Hz}, 2 \mathrm{H}), 7.33-7.29(\mathrm{~m}, 3 \mathrm{H}), 7.28-7.17(\mathrm{~m}, 5 \mathrm{H}), 7.05(\mathrm{~d}, J=6.8 \mathrm{~Hz}, 2 \mathrm{H}), 6.96(\mathrm{~d}, J=7.4$ $\mathrm{Hz}, 2 \mathrm{H}), 4.20$ (s, 2H), 3.92 (s, 2H), 3.77 (s, 2H), 2.34 (s, 3H), 1.14 (s, 12H). ${ }^{13} \mathrm{C}$ NMR (100 MHz, $\left.\mathrm{CDCl}_{3}\right) \delta 156.2,142.5,140.4,139.3,138.9,136.2,129.4,129.1,128.4,128.3,128.1,128.1,127.8$,

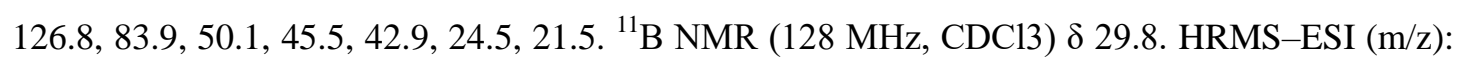
$[\mathrm{M}+\mathrm{H}]^{+}$calcd for $\mathrm{C}_{32} \mathrm{H}_{36} \mathrm{BClNO} \mathrm{S}_{4} \mathrm{~S}, 576.2147$; found: 576.2135 .

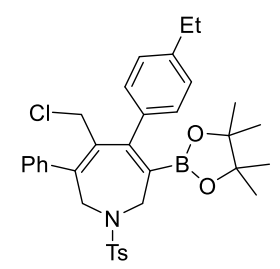

4-(chloromethyl)-5-(4-ethylphenyl)-3-phenyl-6-(4,4,5,5-tetramethyl-1,3,2-dioxaborolan-2-yl)1-tosyl-2,7-dihydro-1H-azepine (4b)

Prepared according to general procedure to give $\mathbf{4 b}(50 \mathrm{mg}, 83 \%)$ as a white solid after short column chromatography (5\%-10\% EtOAc/Petroleum). ${ }^{1} \mathrm{H}$ NMR (400 MHz, $\left.\mathrm{CDCl}_{3}\right) \delta 7.36$ (d, $J=$ $8.3 \mathrm{~Hz}, 2 \mathrm{H}), 7.31-7.21(\mathrm{~m}, 3 \mathrm{H}), 7.17-7.07$ (m, 4H), $7.05(\mathrm{dd}, J=8.1,1.3 \mathrm{~Hz}, 2 \mathrm{H}), 6.96(\mathrm{~d}, J=$ $8.0 \mathrm{~Hz}, 2 \mathrm{H}), 4.19$ (s, 2H), 3.91 (s, 2H), 3.80 (s, 2H), 2.64 (q, J = 7.6 Hz, 2H), 2.34 (s, 3H), 1.22 (t, $J=7.6 \mathrm{~Hz}, 3 \mathrm{H}), 1.15(\mathrm{~s}, 12 \mathrm{H}) .{ }^{13} \mathrm{C} \mathrm{NMR}\left(100 \mathrm{MHz}, \mathrm{CDCl}_{3}\right) \delta 156.1,144.6,142.4,140.3,139.4$, 139.0, 136.3, 136.2, 129.4, 129.0, 128.4, 128.1, 127.8, 127.6, 126.8, 83.9, 50.1, 45.5, 43.0, 28.7, 26.9, 24.5, 21.4, 15.7. ${ }^{11} \mathrm{~B}$ NMR $\left(128 \mathrm{MHz}, \mathrm{CDCl}_{3}\right) \delta 30.3$. HRMS-ESI $(\mathrm{m} / \mathrm{z}):[\mathrm{M}+\mathrm{H}]^{+}$calcd for $\mathrm{C}_{34} \mathrm{H}_{40} \mathrm{BClNO}{ }_{4} \mathrm{~S}, 604.2460$; found: 604.2430 . 


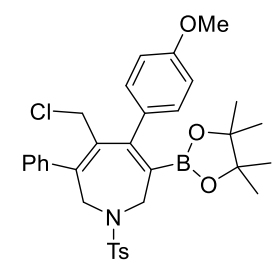

4-(chloromethyl)-5-(4-methoxyphenyl)-3-phenyl-6-(4,4,5,5-tetramethyl-1,3,2-dioxaborolan-2yl)-1-tosyl-2,7-dihydro-1H-azepine (4c)

Prepared according to general procedure to give $4 \mathbf{c}(48 \mathrm{mg}, 79 \%)$ as a white solid after short column chromatography $\left(5 \%-10 \%\right.$ EtOAc/Petroleum). ${ }^{1} \mathrm{H}$ NMR $\left(400 \mathrm{MHz}, \mathrm{CDCl}_{3}\right) \delta 7.36(\mathrm{~d}, J=$ $8.3 \mathrm{~Hz}, 2 \mathrm{H}), 7.29-7.23(\mathrm{~m}, 3 \mathrm{H}), 7.16-7.09(\mathrm{~m}, 2 \mathrm{H}), 7.04(\mathrm{dd}, J=8.1,1.3 \mathrm{~Hz}, 2 \mathrm{H}), 6.96(\mathrm{~d}, J=$ 8.0 Hz, 2H), $6.84(\mathrm{~d}, J=8.7 \mathrm{~Hz}, 2 \mathrm{H}), 4.18(\mathrm{~s}, 2 \mathrm{H}), 3.90(\mathrm{~s}, 2 \mathrm{H}), 3.81(\mathrm{~s}, 3 \mathrm{H}), 3.80(\mathrm{~s}, 2 \mathrm{H}), 2.34(\mathrm{~s}$, 3H), 1.17 (s, 12H). ${ }^{13} \mathrm{C}$ NMR (100 MHz, $\left.\mathrm{CDCl}_{3}\right) \delta 159.8,155.8,142.4,140.4,139.4,139.1,136.2$, $131.4,130.4,129.4,128.4,128.1,127.8,126.8,113.5,83.9,55.3,50.0,45.6,43.0,24.6,21.4 .{ }^{11} \mathrm{~B}$ NMR $\left(128 \mathrm{MHz}, \mathrm{CDCl}_{3}\right) \delta$ 29.7. HRMS-ESI (m/z): $[\mathrm{M}+\mathrm{H}]^{+}$calcd for $\mathrm{C}_{33} \mathrm{H}_{38} \mathrm{BCINO}_{5} \mathrm{~S}, 606.2252$; found: 606.2286 .

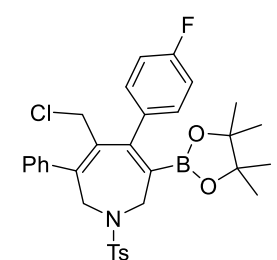

4-(chloromethyl)-5-(4-fluorophenyl)-3-phenyl-6-(4,4,5,5-tetramethyl-1,3,2-dioxaborolan-2-yl) -1-tosyl-2,7-dihydro-1H-azepine (4d)

Prepared according to general procedure to give $\mathbf{4 d}(45 \mathrm{mg}, 76 \%)$ as a white solid after short column chromatography (5\%-10\% EtOAc/Petroleum). ${ }^{1} \mathrm{H}$ NMR $\left(400 \mathrm{MHz}, \mathrm{CDCl}_{3}\right) \delta 7.37(\mathrm{~d}, J=$ $8.3 \mathrm{~Hz}, 2 \mathrm{H}), 7.30-7.17(\mathrm{~m}, 5 \mathrm{H}), 7.06-6.98(\mathrm{~m}, 4 \mathrm{H}), 6.96(\mathrm{~d}, J=8.0 \mathrm{~Hz}, 2 \mathrm{H}), 4.19(\mathrm{~s}, 2 \mathrm{H}), 3.91$ (s, 2H), $3.76(\mathrm{~s}, 2 \mathrm{H}), 2.34(\mathrm{~s}, 3 \mathrm{H}), 1.15$ (s, 12H). ${ }^{13} \mathrm{C} \mathrm{NMR}\left(100 \mathrm{MHz}, \mathrm{CDCl}_{3}\right) \delta$ 164.1, 161.6, $155.3,142.5,140.6,139.2,138.8,136.1,135.0,135.0,130.9,130.8,129.4,128.4,128.1,127.9$, 126.8, 115.1, 114.9, 84.0, 50.0, 45.5, 42.8, 24.5, 21.5. ${ }^{11} \mathrm{~B}$ NMR (128 MHz, $\left.\mathrm{CDCl}_{3}\right) \delta 30.0$. HRMS-ESI (m/z): $[\mathrm{M}+\mathrm{H}]^{+}$calcd for $\mathrm{C}_{32} \mathrm{H}_{35} \mathrm{BClFNO}_{4} \mathrm{~S}$, 594.2052; found: 594.2066.

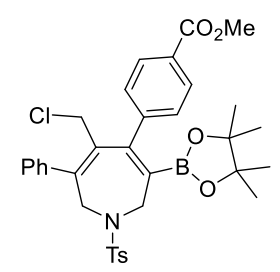

methyl 4-(5-(chloromethyl)-6-phenyl-3-(4,4,5,5-tetramethyl-1,3,2-dioxaborolan-2-yl)-1-tosyl 


\section{-2,7-dihydro-1H-azepin-4-yl)benzoate (4e)}

Prepared according to general procedure to give $4 \mathrm{e}(50 \mathrm{mg}, 79 \%)$ as a white solid after short column chromatography (5\%-10\% EtOAc/Petroleum). ${ }^{1} \mathrm{H}$ NMR $\left(400 \mathrm{MHz}, \mathrm{CDCl}_{3}\right) \delta 7.96(\mathrm{~d}, J=$ $8.4 \mathrm{~Hz}, 2 \mathrm{H}), 7.74(\mathrm{~d}, J=8.3 \mathrm{~Hz}, 2 \mathrm{H}), 7.35-7.28(\mathrm{~m}, 2 \mathrm{H}), 7.26-7.19(\mathrm{~m}, 5 \mathrm{H}), 7.01(\mathrm{~d}, J=8.4$ $\mathrm{Hz}, 2 \mathrm{H}), 4.74(\mathrm{t}, J=2.5 \mathrm{~Hz}, 2 \mathrm{H}), 4.17(\mathrm{~s}, 2 \mathrm{H}), 3.95(\mathrm{~s}, 3 \mathrm{H}), 3.01(\mathrm{t}, J=2.5 \mathrm{~Hz}, 2 \mathrm{H}), 2.42(\mathrm{~s}, 3 \mathrm{H})$, $1.10(\mathrm{~s}, 12 \mathrm{H}) .{ }^{13} \mathrm{C}$ NMR $\left(100 \mathrm{MHz}, \mathrm{CDCl}_{3}\right) \delta 167.1,151.3,147.3,143.7,143.0,142.7,141.6$, 140.5, 137.0, 129.5, 129.1, 128.9, 127.6, 126.7, 126.3, 123.6, 119.1, 83.6, 52.1, 48.4, 48.4, 42.1, 26.9, 24.5, 21.5. ${ }^{11} \mathrm{~B}$ NMR $\left(128 \mathrm{MHz}, \mathrm{CDCl}_{3}\right) \delta$ 29.9. HRMS-ESI $(\mathrm{m} / \mathrm{z}):[\mathrm{M}+\mathrm{H}]^{+}$calcd for $\mathrm{C}_{34} \mathrm{H}_{38} \mathrm{BCINO}_{6} \mathrm{~S}, 634.2201$; found: 634.2233 . 


\section{Derivatization of Borylative Cyclization products}

\subsection{Oxidation of $2 a$}
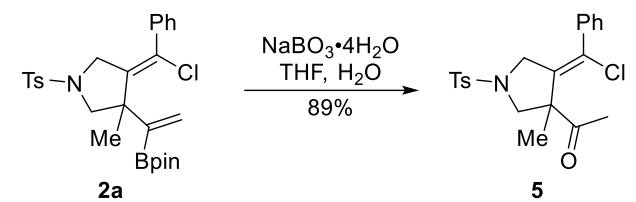

To the solution of borylative cyclization product $\mathbf{2 a}(50 \mathrm{mg}, 0.1 \mathrm{mmol})$ in THF $(2 \mathrm{~mL})$ was added water $(2 \mathrm{~mL})$ and $\mathrm{NaBO}_{3} \cdot 4 \mathrm{H}_{2} \mathrm{O}(65 \mathrm{mg}, 0.3 \mathrm{mmol})$. The resulting mixture was stirred at ambient temperature for $5 \mathrm{~h}$. The resulting mixture was quenched with Sat. $\mathrm{Na}_{2} \mathrm{~S}_{2} \mathrm{O}_{3}$. The mixture was extracted with $\mathrm{Et}_{2} \mathrm{O}$, and the combined organic layer was dried over sodium sulfate and concentrated in vacuo. The crude product was purified by column chromatography on silica gel with $10 \%$ ethyl acetate/petroleum ether as an eluent to give (E)-1-(4-(chloro(phenyl)methylene)-3-methyl-1-tosylpyrrolidin-3-yl)ethan-1-one (5, $36 \mathrm{mg}, 89 \%$ yield $)$ as a yellow oil. ${ }^{1} \mathrm{H}$ NMR $\left(400 \mathrm{MHz}, \mathrm{CDCl}_{3}\right) \delta 7.66(\mathrm{~d}, J=8.3 \mathrm{~Hz}, 2 \mathrm{H}), 7.42-7.33(\mathrm{~m}, 5 \mathrm{H})$, $7.30-7.27(\mathrm{~m}, 2 \mathrm{H}), 3.98(\mathrm{~d}, J=13.6 \mathrm{~Hz}, 1 \mathrm{H}), 3.81(\mathrm{~d}, J=13.6 \mathrm{~Hz}, 1 \mathrm{H}), 3.42(\mathrm{~d}, J=10.1 \mathrm{~Hz}$, 1H), $2.97(\mathrm{~d}, J=10.1 \mathrm{~Hz}, 1 \mathrm{H}), 2.46(\mathrm{~s}, 3 \mathrm{H}), 2.28(\mathrm{~s}, 3 \mathrm{H}), 1.49(\mathrm{~s}, 3 \mathrm{H}) .{ }^{13} \mathrm{C}$ NMR $(100 \mathrm{MHz}$, $\left.\mathrm{CDCl}_{3}\right) \delta 205.8,144.5,137.7,137.6,131.2,130.0,129.4,128.7,128.0,127.7,127.3,58.8,58.3$, 52.9, 26.3, 21.6, 19.3. HRMS-ESI (m/z): $[\mathrm{M}+\mathrm{Na}]^{+}$calcd for $\mathrm{C}_{21} \mathrm{H}_{22} \mathrm{ClNNaO}_{3} \mathrm{~S}, 426.0907$; found: 426.0900 .

\subsection{Suzuki Coupling of 2a with Iodobenzene}

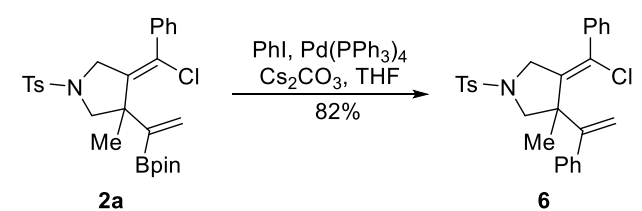

A mixture of vinyl borylative cyclization product $2 \mathbf{a}(50 \mathrm{mg}, 0.1 \mathrm{mmol})$, iodobenzene $(61 \mathrm{mg}, 0.3$ mmol), $\mathrm{Pd}\left(\mathrm{PPh}_{3}\right)_{4}(12 \mathrm{mg}, 0.01 \mathrm{mmol})$, and $\mathrm{Cs}_{2} \mathrm{CO}_{3}(98 \mathrm{mg}, 0.3 \mathrm{mmol})$ in $\mathrm{THF}(5 \mathrm{~mL})$ was heated at $60{ }^{\circ} \mathrm{C}$ for $24 \mathrm{~h}$. After cooling of the mixture to room temperature, water was added and mixture was extracted with EtOAc. The extracts were dried over anhydrous $\mathrm{Na}_{2} \mathrm{SO}_{4}$ and concentrated in vacuo. The crude product was purified by column chromatography on silica gel with $10 \%$ ethyl acetate/petroleum ether as an eluent to give (E)-4-(chloro(phenyl)methylene)-3-methyl-3-(1-phenylvinyl)-1-tosylpyrrolidine $(6,38 \mathrm{mg}, 82 \%$ yield) as a yellow oil. ${ }^{1} \mathrm{H}$ NMR $\left(400 \mathrm{MHz}, \mathrm{CDCl}_{3}\right) \delta 7.55(\mathrm{~d}, J=8.2 \mathrm{~Hz}, 2 \mathrm{H}), 7.38-7.27(\mathrm{~m}, 5 \mathrm{H})$, 
$7.24-7.06(\mathrm{~m}, 7 \mathrm{H}), 5.40(\mathrm{~s}, 1 \mathrm{H}), 5.20(\mathrm{~s}, 1 \mathrm{H}), 3.83(\mathrm{~d}, J=13.9 \mathrm{~Hz}, 1 \mathrm{H}), 3.46(\mathrm{~s}, 1 \mathrm{H}), 3.33(\mathrm{~d}, J=$ $9.5 \mathrm{~Hz}, 1 \mathrm{H}), 3.20(\mathrm{~d}, J=9.4 \mathrm{~Hz}, 1 \mathrm{H}), 2.45(\mathrm{~s}, 3 \mathrm{H}), 1.68(\mathrm{~s}, 3 \mathrm{H}) .{ }^{13} \mathrm{C}$ NMR $\left(100 \mathrm{MHz}, \mathrm{CDCl}_{3}\right) \delta$ $150.0,143.9,141.5,139.1,139.1,131.1,129.6,128.8,128.7,128.4,128.0,127.7,127.6,127.0$, 126.1, 116.6, 61.4, 54.0, 52.0, 22.8, 21.6. HRMS-ESI (m/z): $[\mathrm{M}+\mathrm{H}]^{+}$calcd for $\mathrm{C}_{27} \mathrm{H}_{27} \mathrm{ClNO}_{2} \mathrm{~S}$, 464.1451; found: 464.1467 .

\subsection{The preparation of potassium fluoborate}

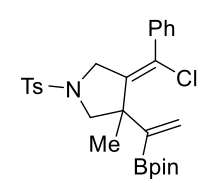

2a

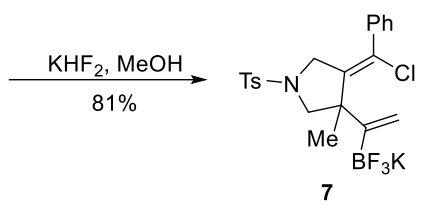

To a solution of vinyl borylative cyclization product $\mathbf{2 a}(50 \mathrm{mg}, 0.1 \mathrm{mmol})$ in methanol $(0.5 \mathrm{~mL})$ was added $\mathrm{KHF}_{2}(0.02 \mathrm{~mL}, 4.5 \mathrm{M}$ saturated aqueous solution, $4.5 \mathrm{mmol}, 1.0$ equiv) dropwise. The reaction mixture stirred at room temperature for $30 \mathrm{~min}$. The solvent was then removed under vacuum and the solid residue was triturated with dry acetone $(0.5 \mathrm{~mL})$. The liquid phase was carefully decanted, and the residual inorganic salts were washed with additional acetone $(3 \times 0.2 \mathrm{~mL})$. The combined solution was concentrated in vacuo to give white solids. The solids was washed with ether $(3 \times 0.5 \mathrm{~mL})$ to remove pinacol and dried under vacuum, affording the desired product (E)-4-(chloro(phenyl)methylene)-3-methyl-1-tosyl-3-(1-(trifluoro-14-boranyl)vinyl) pyrrolidine, potassium salt $(7,40 \mathrm{mg}, 81 \%)$ as a white solid. ${ }^{1} \mathrm{H}$ NMR $\left(400 \mathrm{MHz}, \mathrm{CD}_{3} \mathrm{OD}\right) \delta 7.30$ $(\mathrm{d}, J=8.2 \mathrm{~Hz}, 2 \mathrm{H}), 7.15-7.02(\mathrm{~m}, 5 \mathrm{H}), 7.01-6.95(\mathrm{~m}, 2 \mathrm{H}), 5.03(\mathrm{~d}, J=3.4 \mathrm{~Hz}, 1 \mathrm{H}), 4.62(\mathrm{~s}$, 2H), $3.13(\mathrm{~d}, J=9.1 \mathrm{~Hz}, 1 \mathrm{H}), 3.03(\mathrm{dd}, J=3.1,1.5 \mathrm{~Hz}, 1 \mathrm{H}), 2.73(\mathrm{~d}, J=9.1 \mathrm{~Hz}, 1 \mathrm{H}), 2.15(\mathrm{~s}, 3 \mathrm{H})$, $1.31(\mathrm{~s}, 3 \mathrm{H}) .{ }^{13} \mathrm{C}$ NMR (100 MHz, $\left.\mathrm{CD}_{3} \mathrm{OD}\right) \delta 144.8,142.1,140.9,132.3,130.1,128.9,128.8$, 128.4, 124.7, 116.0, 63.1, 53.1, 30.1, 22.1, 20.9. ${ }^{11}$ B NMR (128 MHz, $\left.\mathrm{CD}_{3} \mathrm{OD}\right) \delta$ 4.0. HRMS-ESI (m/z): $[\mathrm{M}-\mathrm{K}]^{-}$calcd for $\mathrm{C}_{21} \mathrm{H}_{21} \mathrm{BClF}_{3} \mathrm{NO}_{2} \mathrm{~S}, 454.1027$; found: 454.1077.

\subsection{Suzuki Coupling of 4a with 1-iodo-4-methylbenzene}

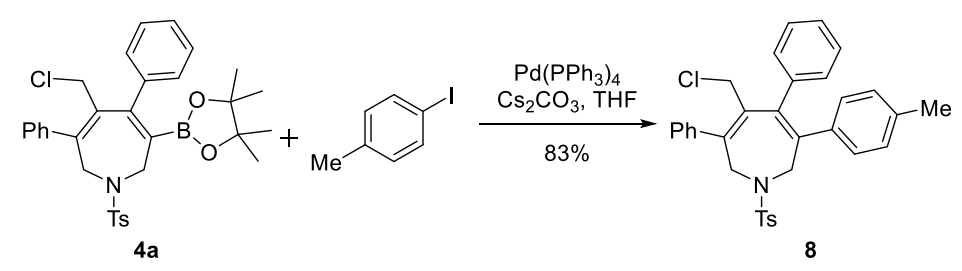

A mixture of vinyl borylative cyclization product $4 \mathbf{a}(56 \mathrm{mg}, 0.1 \mathrm{mmol})$, 1-iodo-4-methylbenzene (65 mg, $0.3 \mathrm{mmol}), \mathrm{Pd}\left(\mathrm{PPh}_{3}\right)_{4}(12 \mathrm{mg}, 0.01 \mathrm{mmol})$, and $\mathrm{Cs}_{2} \mathrm{CO}_{3}(98 \mathrm{mg}, 0.3 \mathrm{mmol})$ in THF (5 mL) was heated at $60{ }^{\circ} \mathrm{C}$ for $24 \mathrm{~h}$. After cooling of the mixture to room temperature, water was added 
and mixture was extracted with EtOAc. The extracts were dried over anhydrous $\mathrm{Na}_{2} \mathrm{SO}_{4}$ and concentrated in vacuo. The crude product was purified by column chromatography on silica gel with $10 \%$ ethyl acetate/petroleum ether as an eluent to give 4-(chloromethyl)-3,5-diphenyl-6-(p-tolyl)-1-tosyl-2,7-dihydro-1H-azepine (8, $45 \mathrm{mg}, 83 \%$ yield) as a yellow oil. ${ }^{1} \mathrm{H} \mathrm{NMR}\left(400 \mathrm{MHz}, \mathrm{CDCl}_{3}\right) \delta 7.38-7.33(\mathrm{~m}, 3 \mathrm{H}), 7.32-7.27(\mathrm{~m}, 2 \mathrm{H}), 7.21(\mathrm{~d}, J$ $=8.0 \mathrm{~Hz}, 2 \mathrm{H}), 7.17-7.11(\mathrm{~m}, 3 \mathrm{H}), 6.94(\mathrm{dd}, J=6.1,2.6 \mathrm{~Hz}, 2 \mathrm{H}), 6.82(\mathrm{~d}, J=7.9 \mathrm{~Hz}, 2 \mathrm{H}), 6.78$ $(\mathrm{d}, J=8.0 \mathrm{~Hz}, 2 \mathrm{H}), 6.68(\mathrm{~d}, J=7.9 \mathrm{~Hz}, 2 \mathrm{H}), 4.36(\mathrm{~s}, 2 \mathrm{H}), 4.19(\mathrm{~s}, 2 \mathrm{H}), 3.88(\mathrm{~s}, 2 \mathrm{H}), 2.28(\mathrm{~s}, 3 \mathrm{H})$, 2.26 (s, 3H). ${ }^{13} \mathrm{C} \mathrm{NMR}\left(100 \mathrm{MHz}, \mathrm{CDCl}_{3}\right) \delta 142.4,141.4,139.5,139.4,138.8,137.5,137.3,136.9$, $136.6,135.3,130.3,129.3,129.2,128.6,128.5,128.3,128.2,128.0,127.4,126.6,50.6,50.3,42.6$, 21.4, 21.2. HRMS-ESI (m/z): $[\mathrm{M}+\mathrm{H}]^{+}$calcd for $\mathrm{C}_{33} \mathrm{H}_{31} \mathrm{ClNO}_{2} \mathrm{~S}, 540.1764$; found: 540.1760 .

\section{Calculation results}

Gaussian 09 program suit was employed to carry out quantum chemical calculations ${ }^{2}$. All the geometrical structures were optimized at the PBE0 level with Grimme's dispersion correction at the D3 level (PBE0-D3), and the def2-SVP basis sets were used for all the atoms. Frequency analyses were done at the same level. For all the reactants, products and mediums, there are no imaginary frequencies. While, for transition states, there are only one imaginary frequency. Relative energies include electronic and zero-point vibrational energies. All positive frequencies that are less than $100 \mathrm{~cm}^{-1}$ are set to $100 \mathrm{~cm}^{-1}$ for thermodynamics calculations ${ }^{3}$. For solvation effects, SMD continuum solvation model was adopted. And the solvation free energies were calculated at M05-2X/6-31G* level on the PBE0-D3/def2-SVP structures. Previous studies have also demonstrated that the M05-2X/6-31G* method combined with SMD model is a good choice to obtain solvation free energy ${ }^{4}$.

To elucidate the product selectivity of different rings formed for different substituent groups, we have performed theoretical studies by density theory functional (DFT) methods. The calculated results are shown in Fig. S1 and S2. Overall, the reactions proceed in several steps and the step from substrates (1a and $\mathrm{BCl}_{3}$ or $\mathbf{3 a}$ and $\mathrm{BCl}_{3}$ ) to mediums (M1 or $\mathrm{M} 2$ ) is rare-determining step. For reactant 1a (Fig. S1), which has a methyl group, according to pre-equilibrium approximation in transition state theory, pathway for formation of five-membered ring has a Gibbs free energy 
barrier of $23.30 \mathrm{~kJ} / \mathrm{mol}$. In contrast, seven-membered ring reaction pathway has a barrier of 95.00 $\mathrm{kJ} / \mathrm{mol}$, which could not be proceeded so smoothly as experimental conditions (room temperature). So, for reactant 1a, product with five-membered ring (FP1) has only observed in our experiment. For reactant 3a (Fig. S2), which has a benzene group, the barriers for formation of five-membered ring (FP2) and seven-membered ring products (SP2) are 46.26 and $89.12 \mathrm{~kJ} / \mathrm{mol}$, respectively. And by the prediction of classical transition state theory, the half-life of the reaction for seven-membered ring pathway is about $7.5 \mathrm{~min}$. Therefore, for reactant 3a, the two pathways both are feasible from the calculated barriers. Furthermore, we scanned the forming process of SR2 and FR2, respectively (shown in Fig. S3). It seems that SR2 is more stable and more easily formed. So, once SR2 is produced, it could quickly generate the final product SP2.

In addition, we also noted that for product with five-membered ring, reactant 1a with the methyl group has a lower barrier than reactant 3a with the benzene group. Considering the reactive mechanism of five-membered ring formation is an electrophilic addition process, the carbon atom attacking $\mathrm{C} \equiv \mathrm{C}$ with more positive charge (shown in red circle in Fig. S1) will accelerate the reaction. The carbon atom in reactants 1a and 3a has natural charges of 0.184 and 0.138, respectively. So, reactant 1a with five-membered ring formed is more favorable.

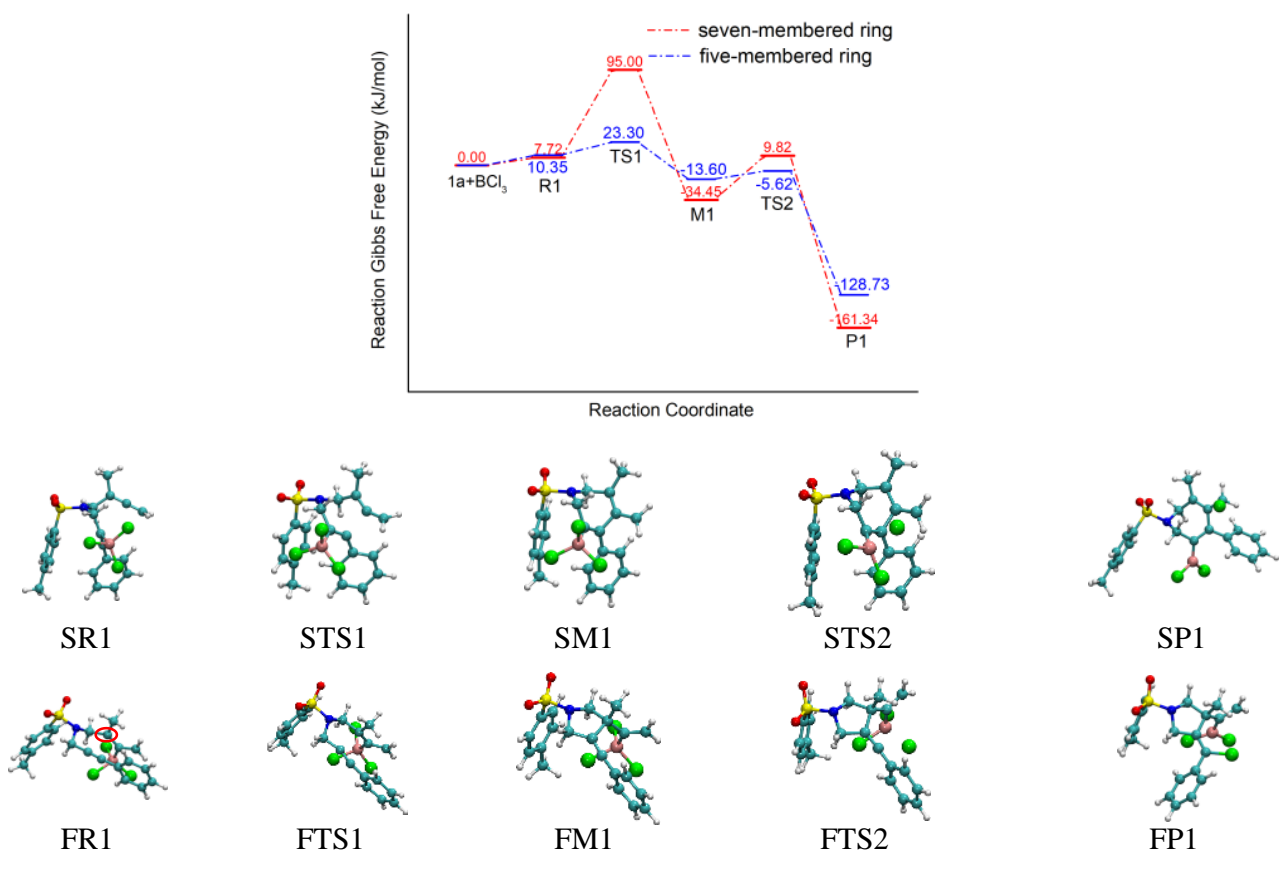

Figure S1. Calculated two different mechanisms for reactant 1a. SX and FX (X=R1, TS1, M1, TS2, P1) donate optimized structures of seven-membered ring and 
five-membered ring pathway, respectively. Reaction Gibbs free energy includes Gibbs free energy calculated in the gas phase and solvation free energy.

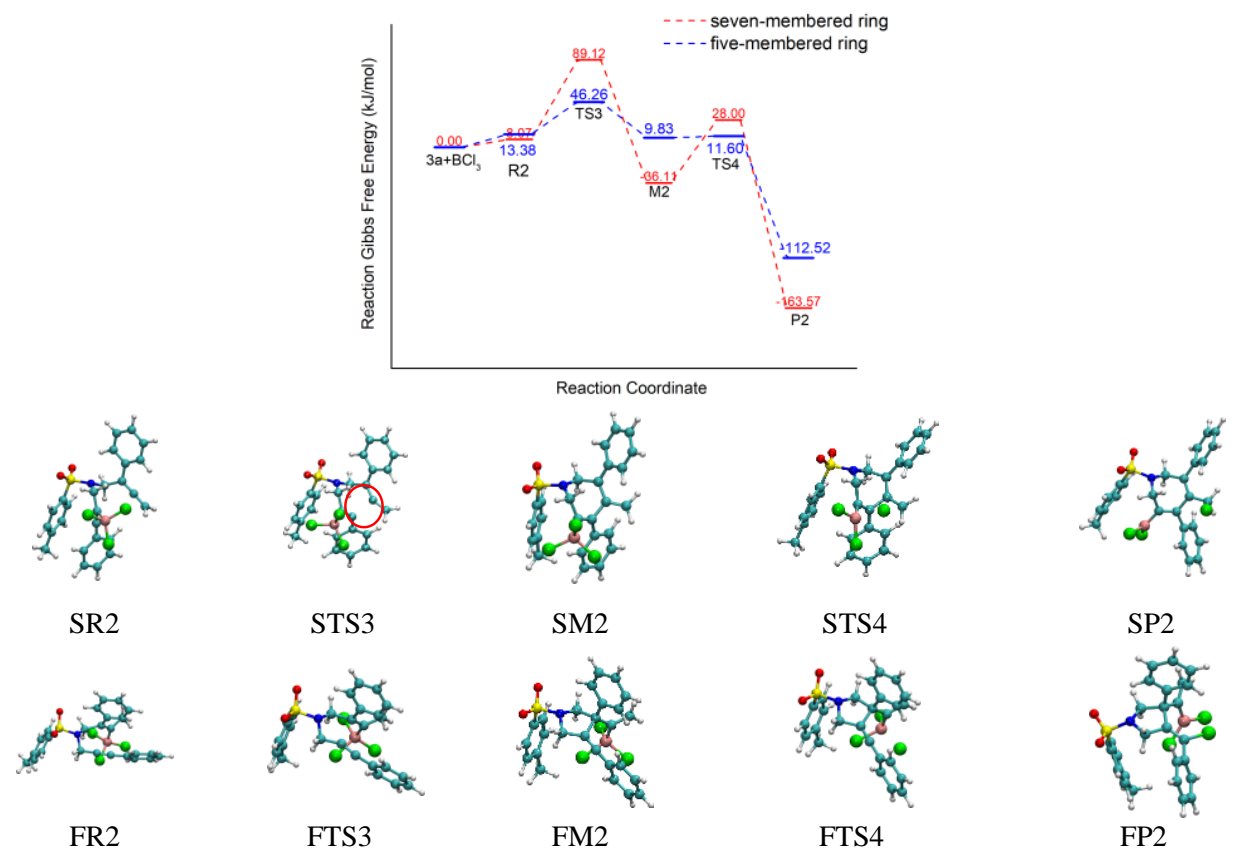

Figure S2. Calculated two different mechanisms for reactant 3a. SX and FX (X=R2, TS3, M2, TS4, P2) donate optimized structures of seven-membered ring and five-membered ring pathway, respectively. Reaction Gibbs free energy includes Gibbs free energy calculated in the gas phase and solvation free energy.

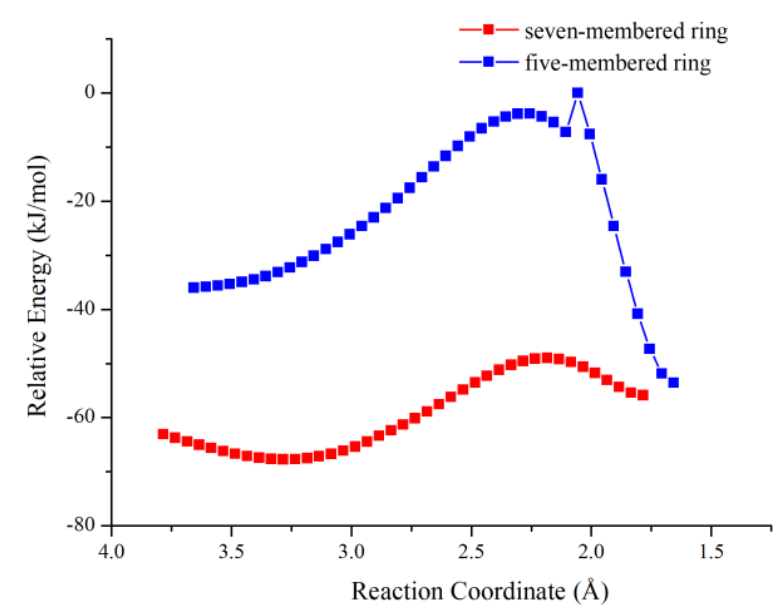

Figure S3. Relaxed scan of potential energy surface for $\mathrm{BCl}_{3}$ approaching to substrate to form SR2 (red curve) and FR2 (blue curve), respectively. Relative energies are only comprised of electronic energies. 


\section{References}

(1) J. H. Park, S.Y. Kim, S. M. Kim, S. I. Lee, Y. K.Chung, Synlett 2007, (3), 453-459.

(2) M. J. Frisch, G. W. Trucks, H. B. Schlegel, G. E. Scuseria, M. A. Robb, J. R. Cheeseman, G. Scalmani, V. Barone, B. Mennucci, G. A. Petersson, H. Nakatsuji, M. Caricato, X. Li, H. P. Hratchian, A. F. Izmaylov, J. Bloino, G. Zheng, J. L. Sonnenberg, M. Hada, M. Ehara, K. Toyota, R. Fukuda, J. Hasegawa, M. Ishida, T. Nakajima, Y. Honda, O. Kitao, H. Nakai, T. Vreven, J. J. A. Montgomery, J. E. Peralta, F. Ogliaro, M. Bearpark, J. J. Heyd, E. Brothers, K. N. Kudin, V. N. Staroverov, T. Keith, R. Kobayashi, J. Normand, K. Raghavachari, A. Rendell, J. C. Burant, S. S. Iyengar, J. Tomasi, M. Cossi, N. Rega, J. M. Millam, M. Klene, J. E. Knox, J. B. Cross, V. Bakken, C. Adamo, J. Jaramillo, R. Gomperts, R. E. Stratmann, O. Yazyev, A. J. Austin, R. Cammi, C. Pomelli, J. W. Ochterski, R. L. Martin, K. Morokuma, V. G. Zakrzewski, G. A. Voth, P. Salvador, J. J. Dannenberg, S. Dapprich, A. D. Daniels, O. Farkas, J. B. Foresman, J. V. Ortiz, J. Cioslowski, and D. J. Fox, Gaussian 09, Revision D.01, Wallingford CT: Gaussian, Inc. 2013.

(3) T. Lu, Q. Chen, Comput. Theor. Chem., 2021, 1200, 113249.

(4) J. Ho, A. Klamt, and M. L. Coote, J. Phys. Chem. A 2010, 114, 13442-13444. 


\section{Copies of NMR Spectra}

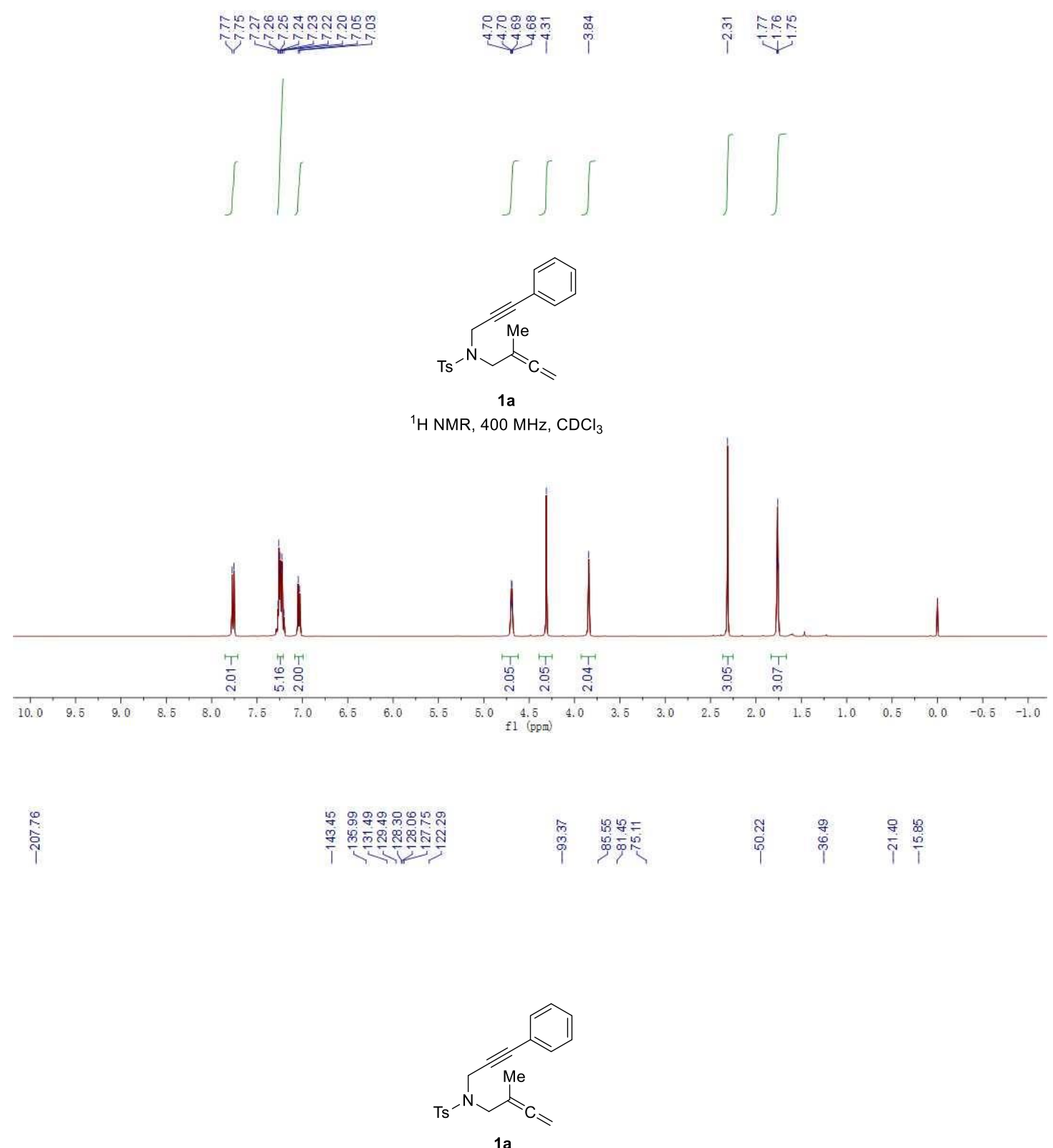

${ }^{13} \mathrm{C} \mathrm{NMR}, 100 \mathrm{MHz}, \mathrm{CDCl}_{3}$

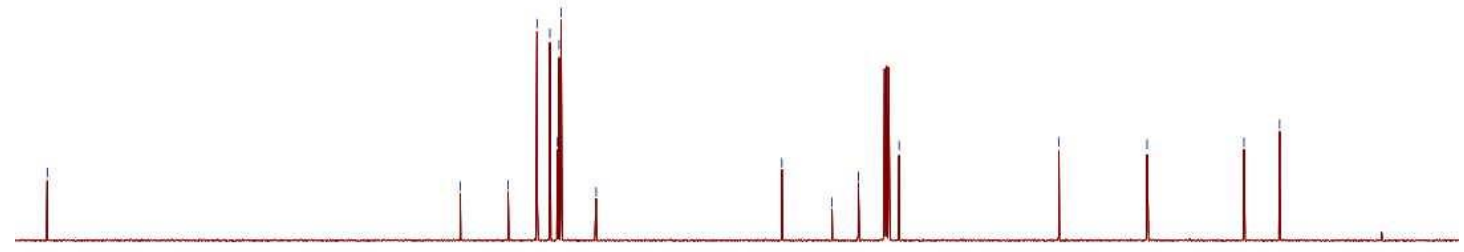

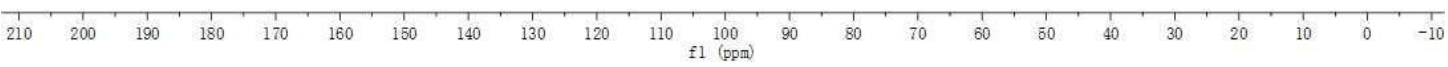




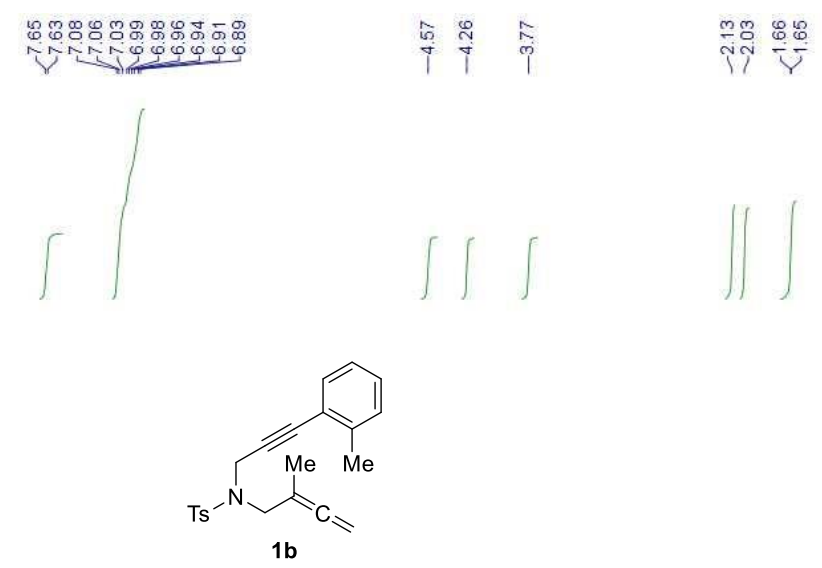

${ }^{1} \mathrm{H} \mathrm{NMR}, 400 \mathrm{MHz}, \mathrm{CDCl}_{3}$

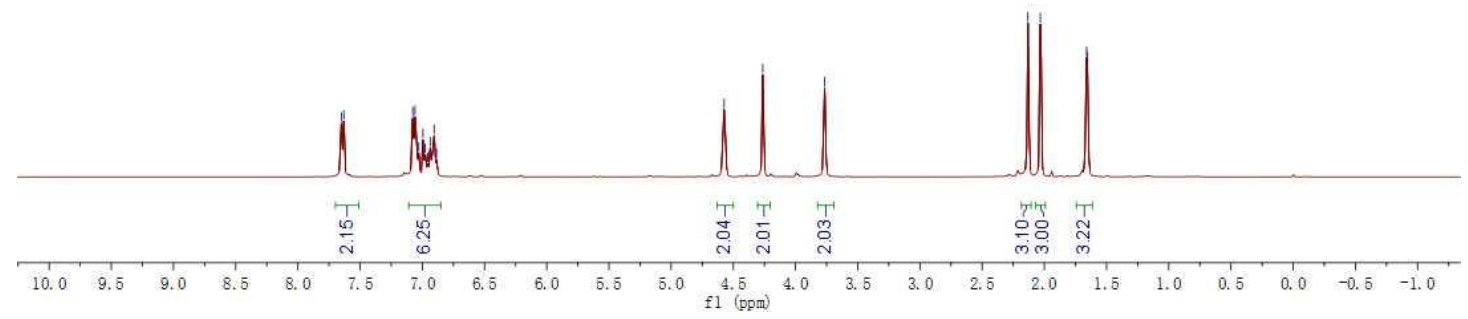

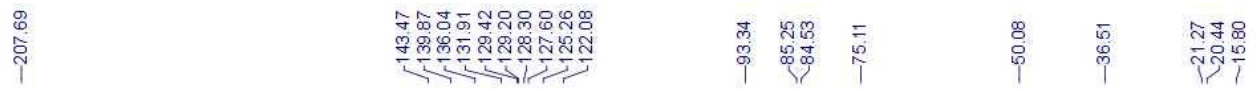

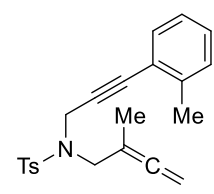

$1 b$

${ }^{13} \mathrm{C} \mathrm{NMR,} 100 \mathrm{MHz}, \mathrm{CDCl}_{3}$

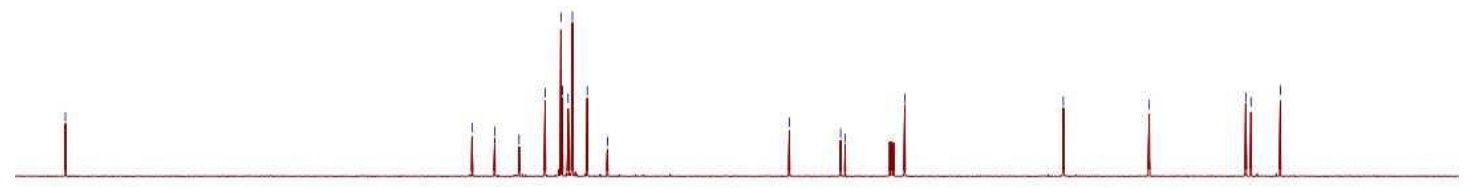

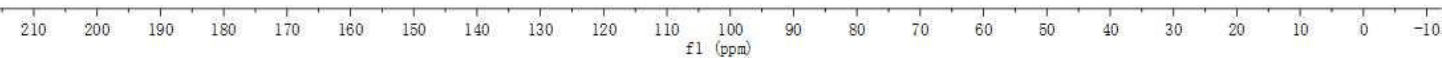




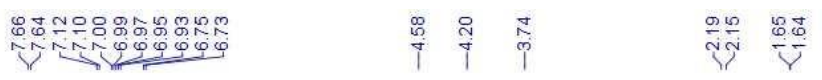
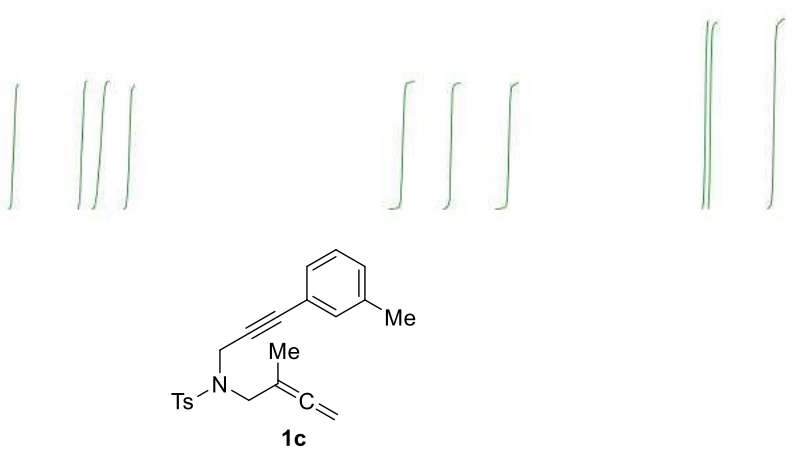

${ }^{1} \mathrm{H} \mathrm{NMR}, 400 \mathrm{MHz}, \mathrm{CDCl}_{3}$
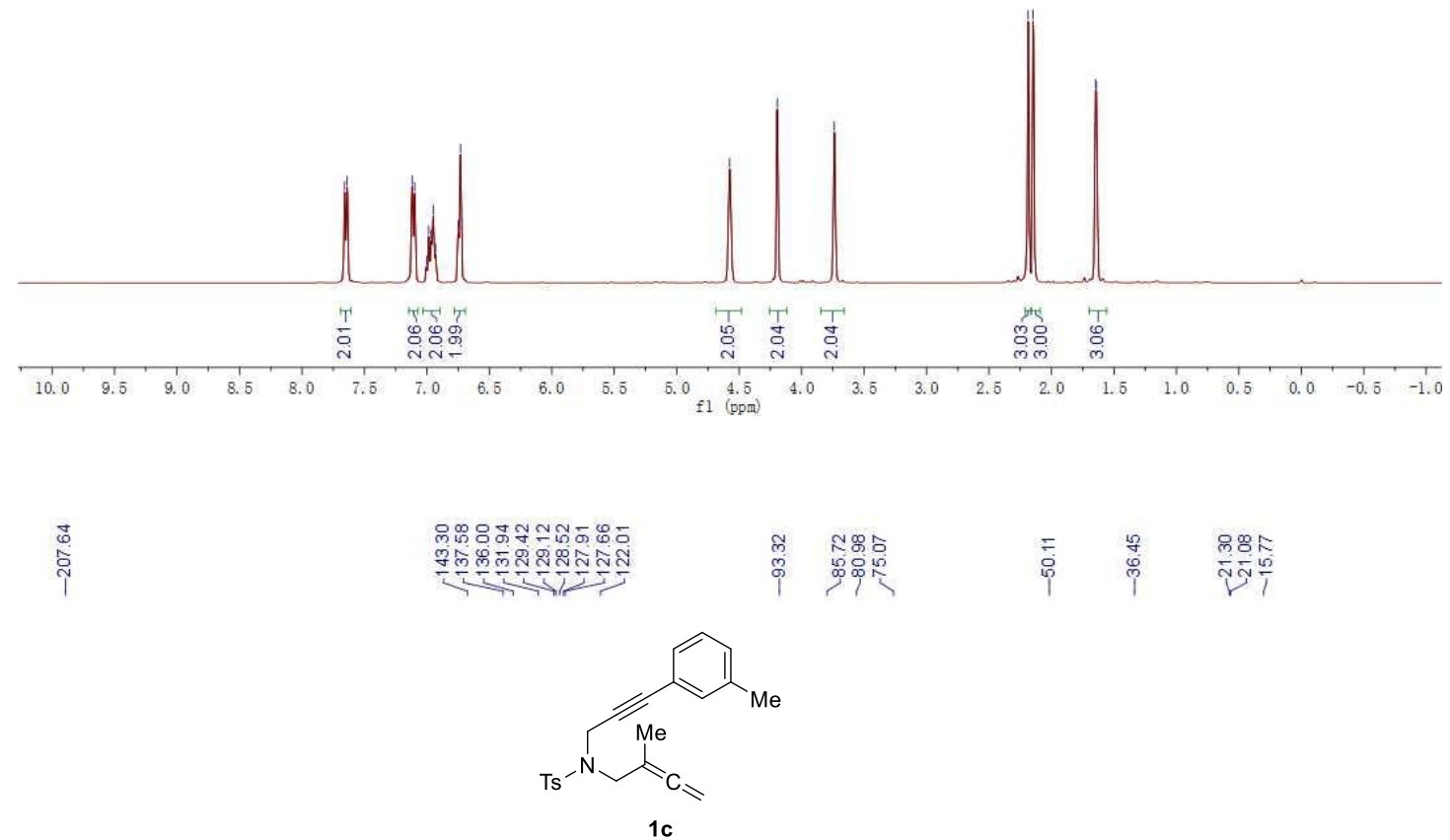

${ }^{13} \mathrm{C}$ NMR, $100 \mathrm{MHz}, \mathrm{CDCl}_{3}$

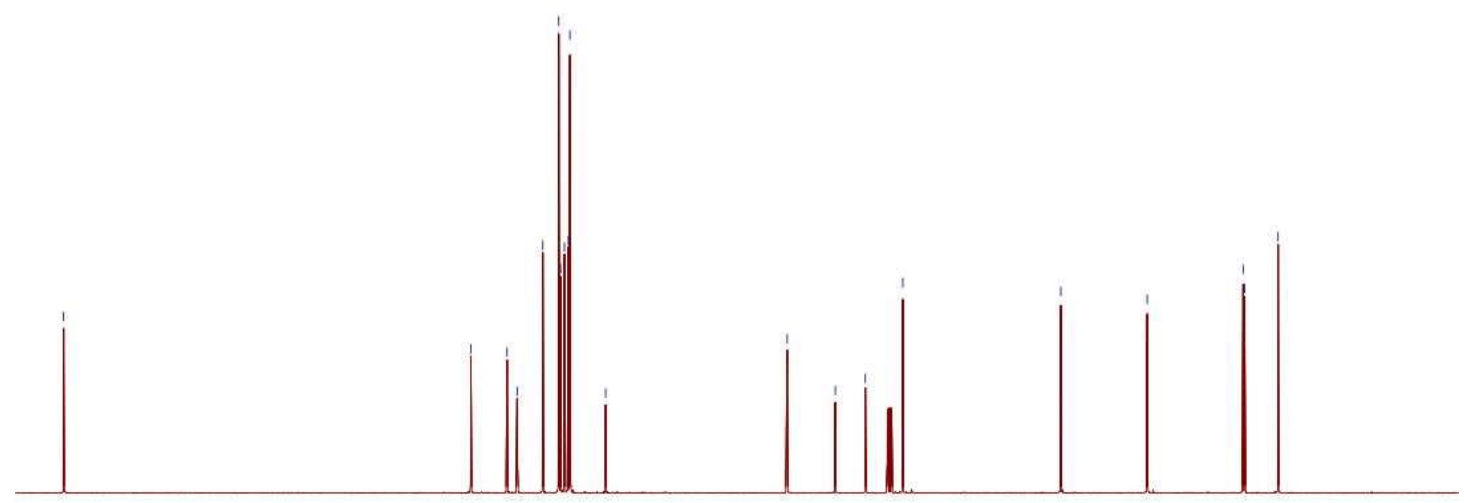

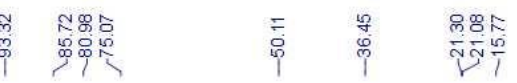

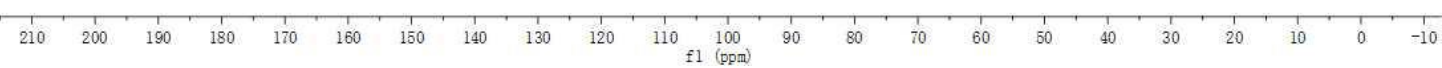



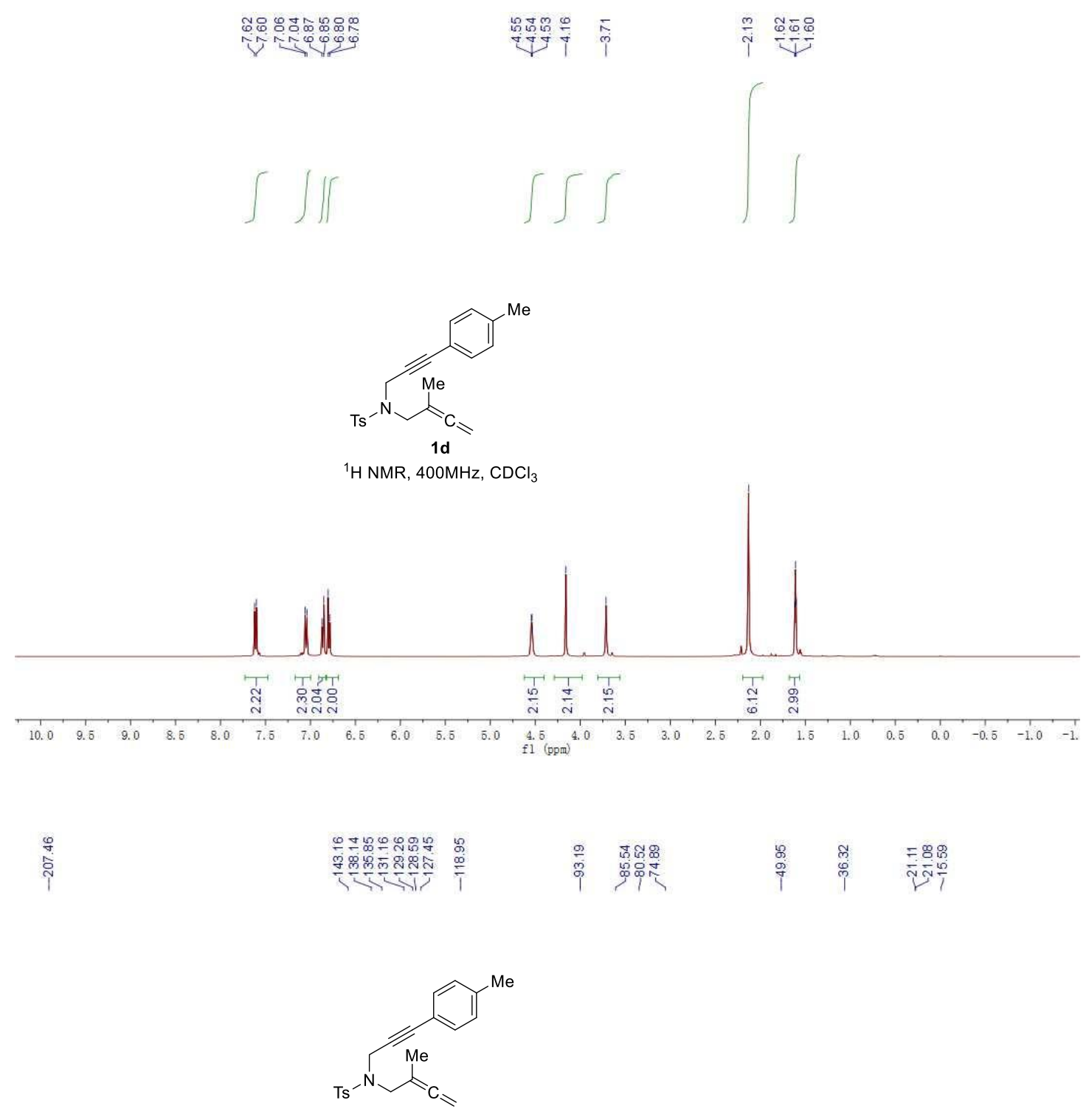

${ }^{13} \mathrm{C} \mathrm{NMR,} 100 \mathrm{MHz}, \mathrm{CDCl}_{3}$

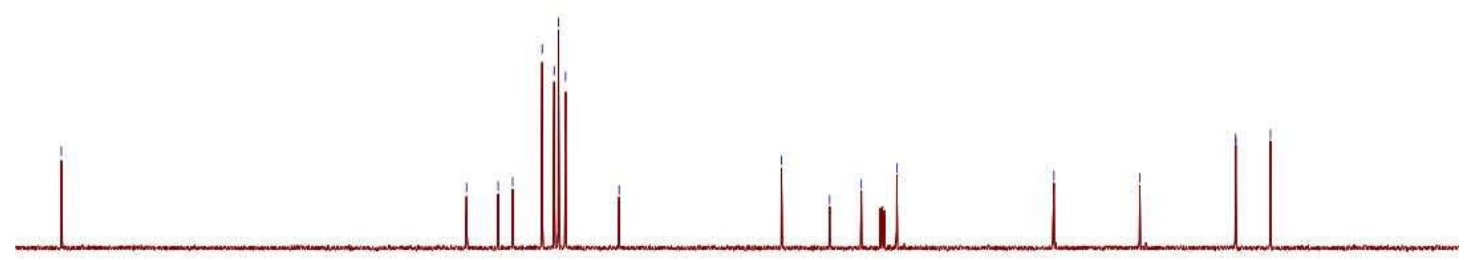

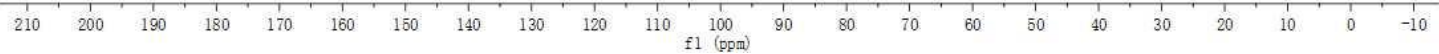



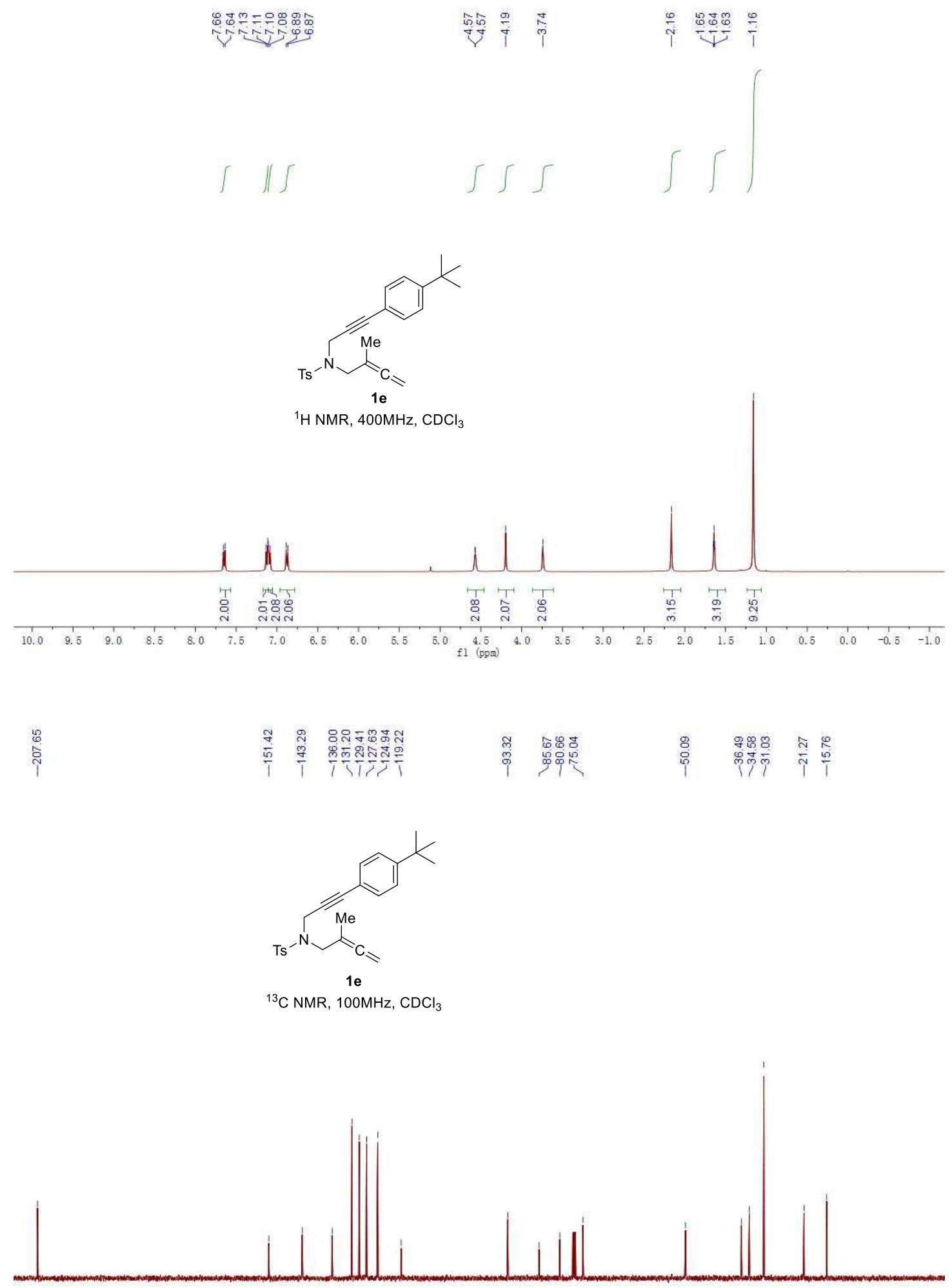

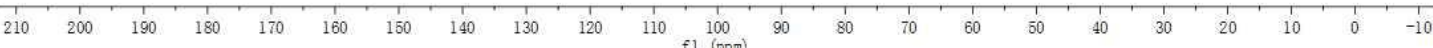



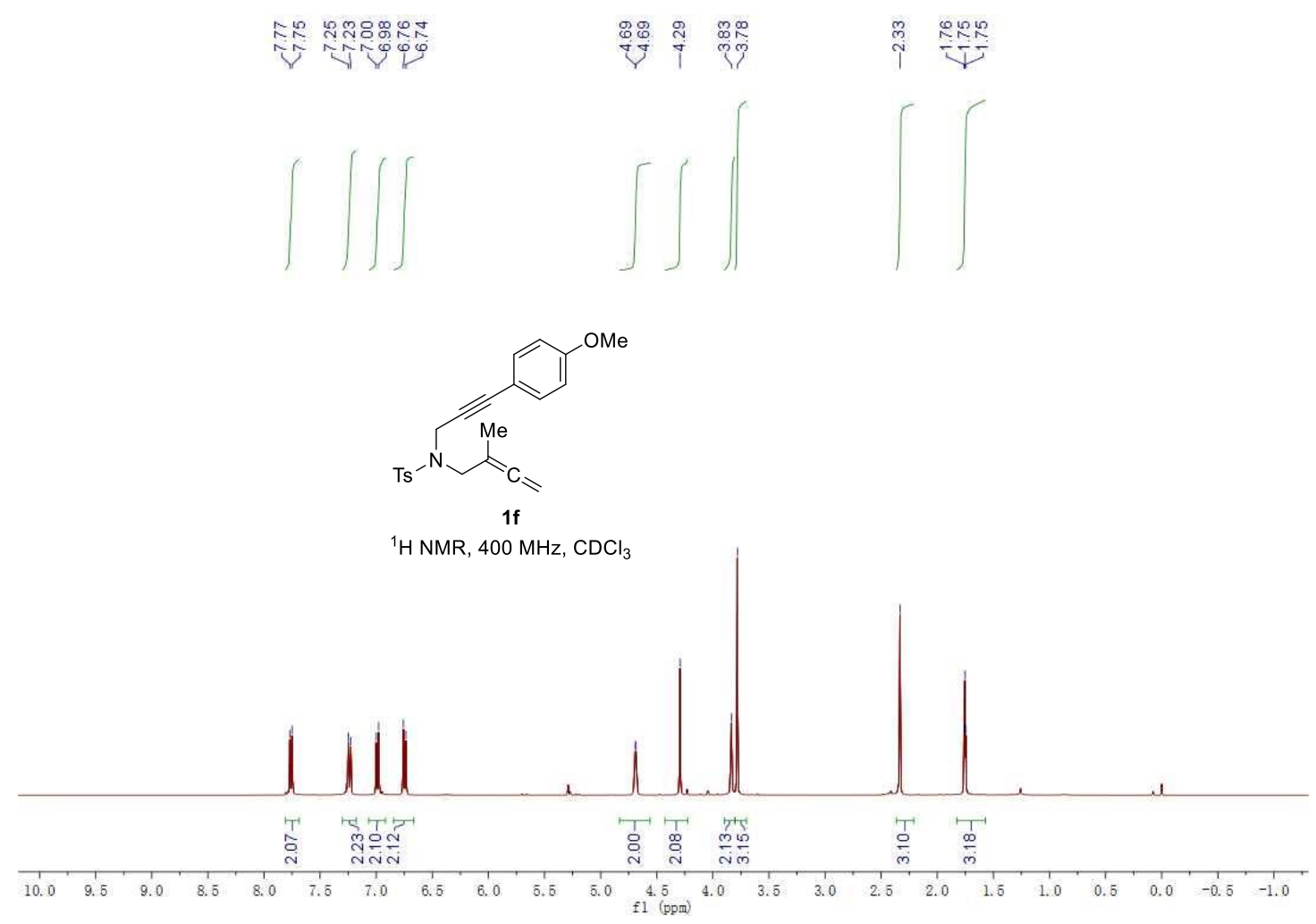

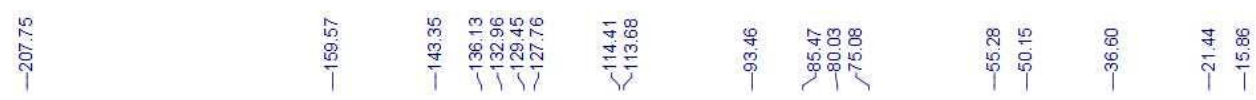
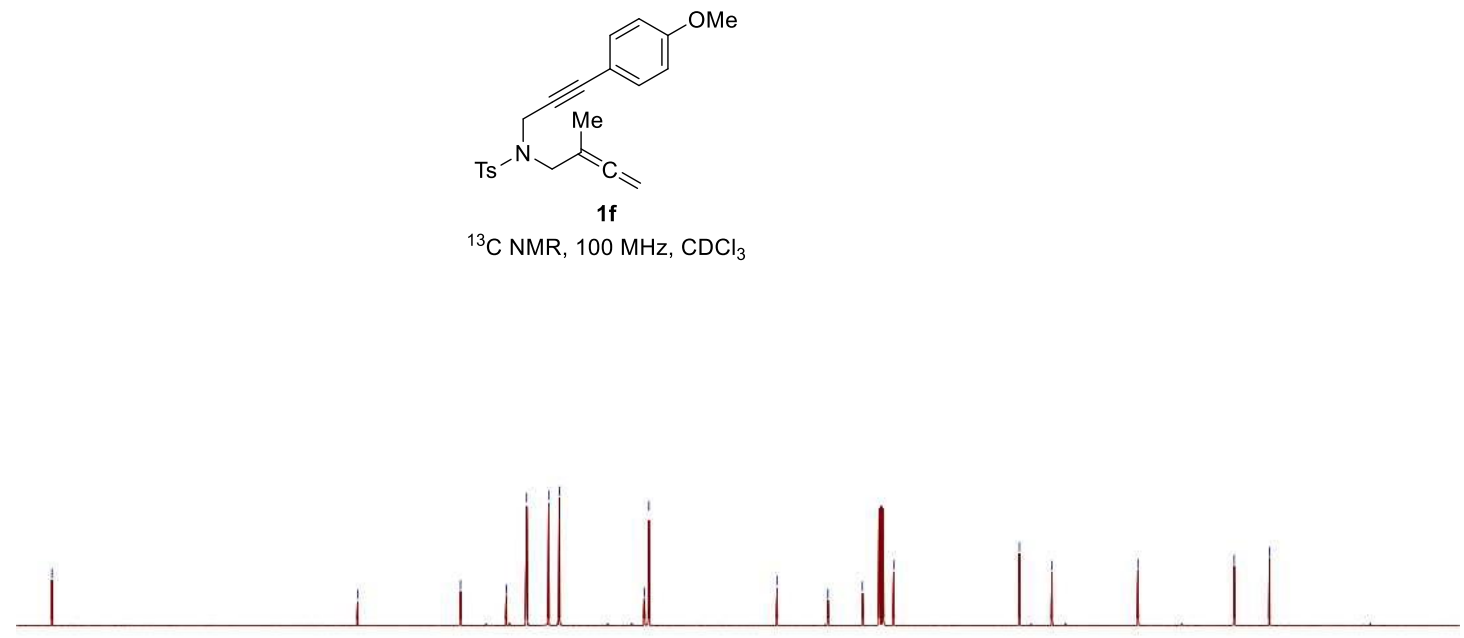

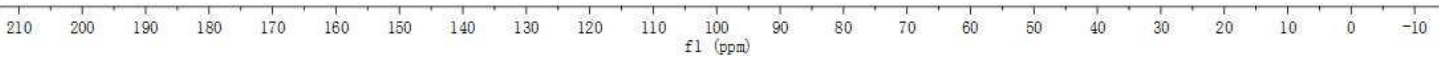




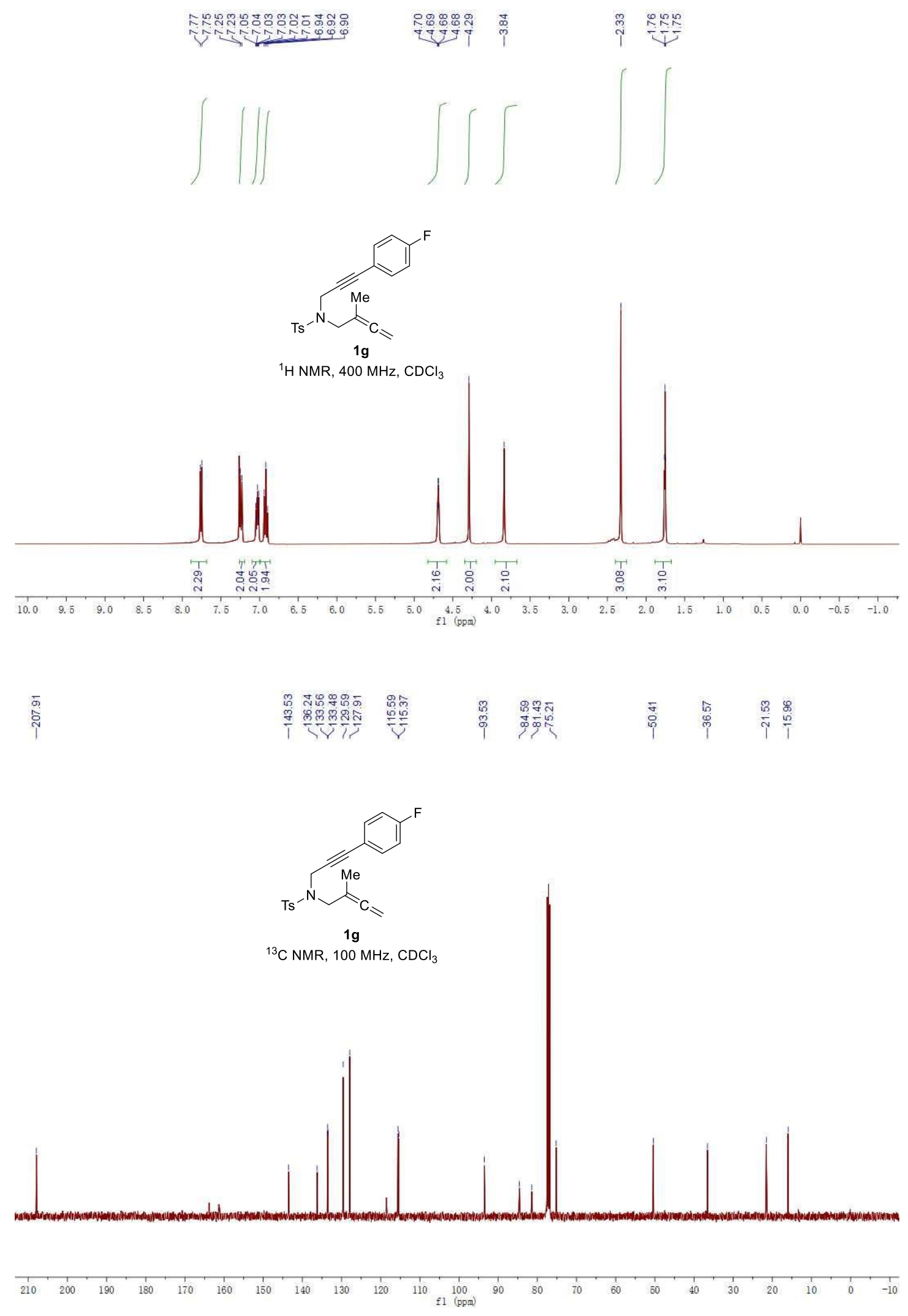




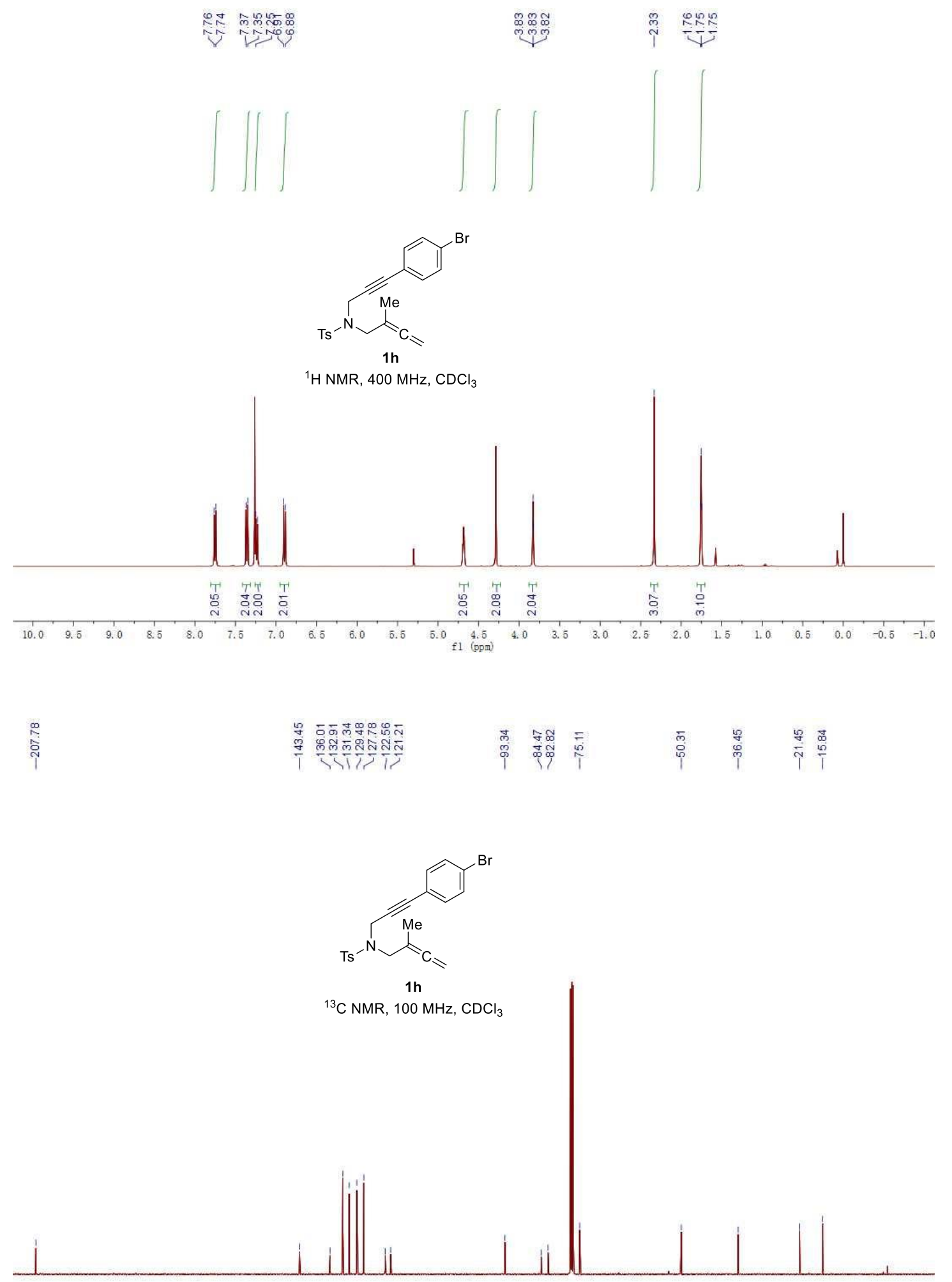

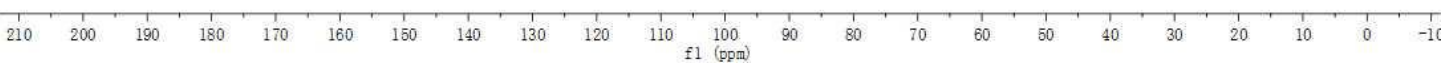




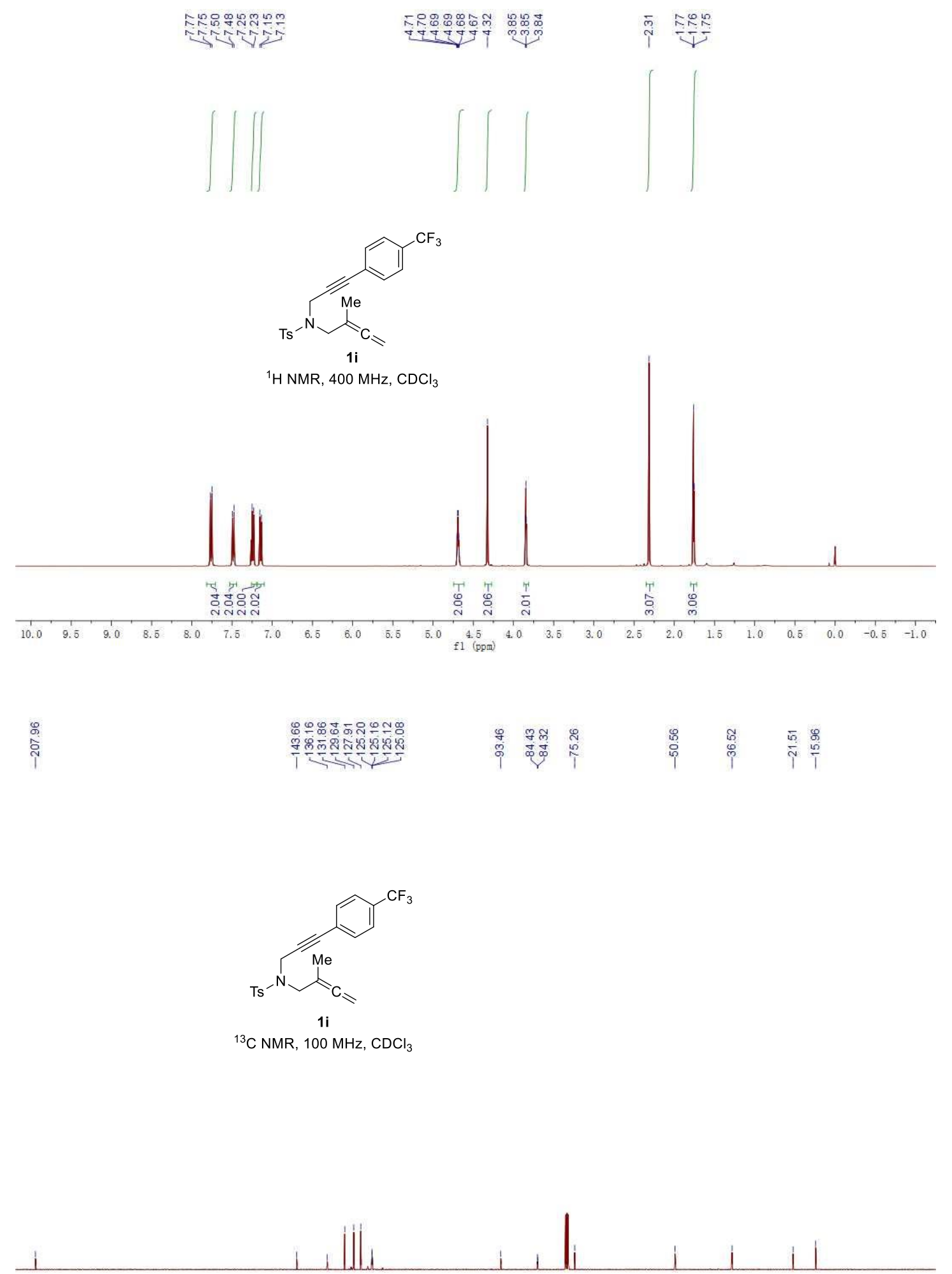

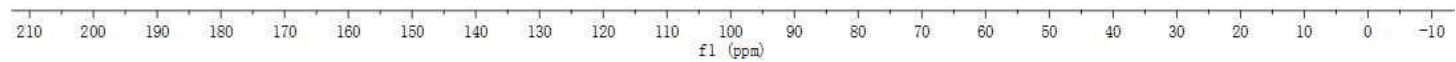



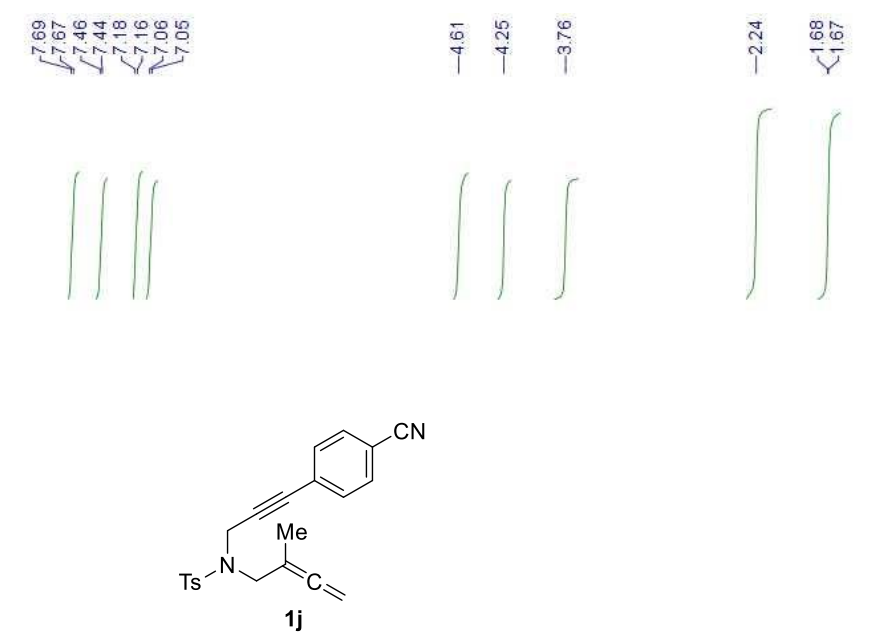

${ }^{1} \mathrm{H}$ NMR, $400 \mathrm{MHz}, \mathrm{CDCl}_{3}$
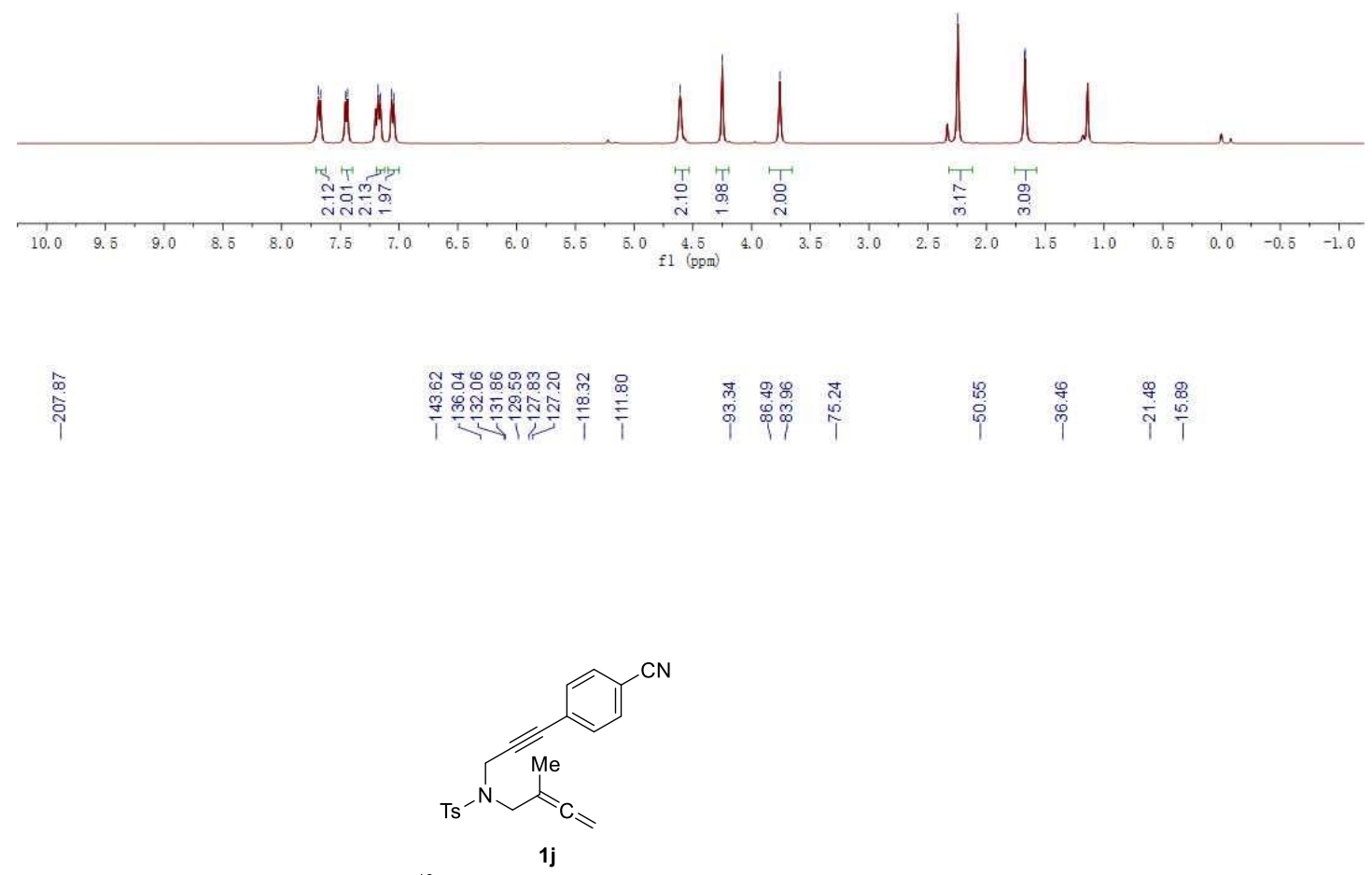

${ }^{13} \mathrm{C} \mathrm{NMR,} 100 \mathrm{MHz}, \mathrm{CDCl}_{3}$

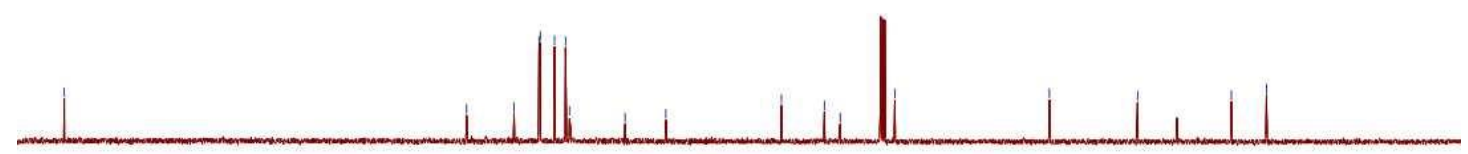

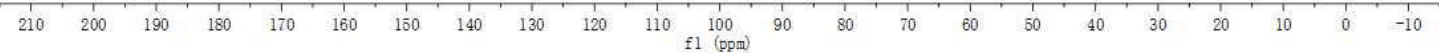




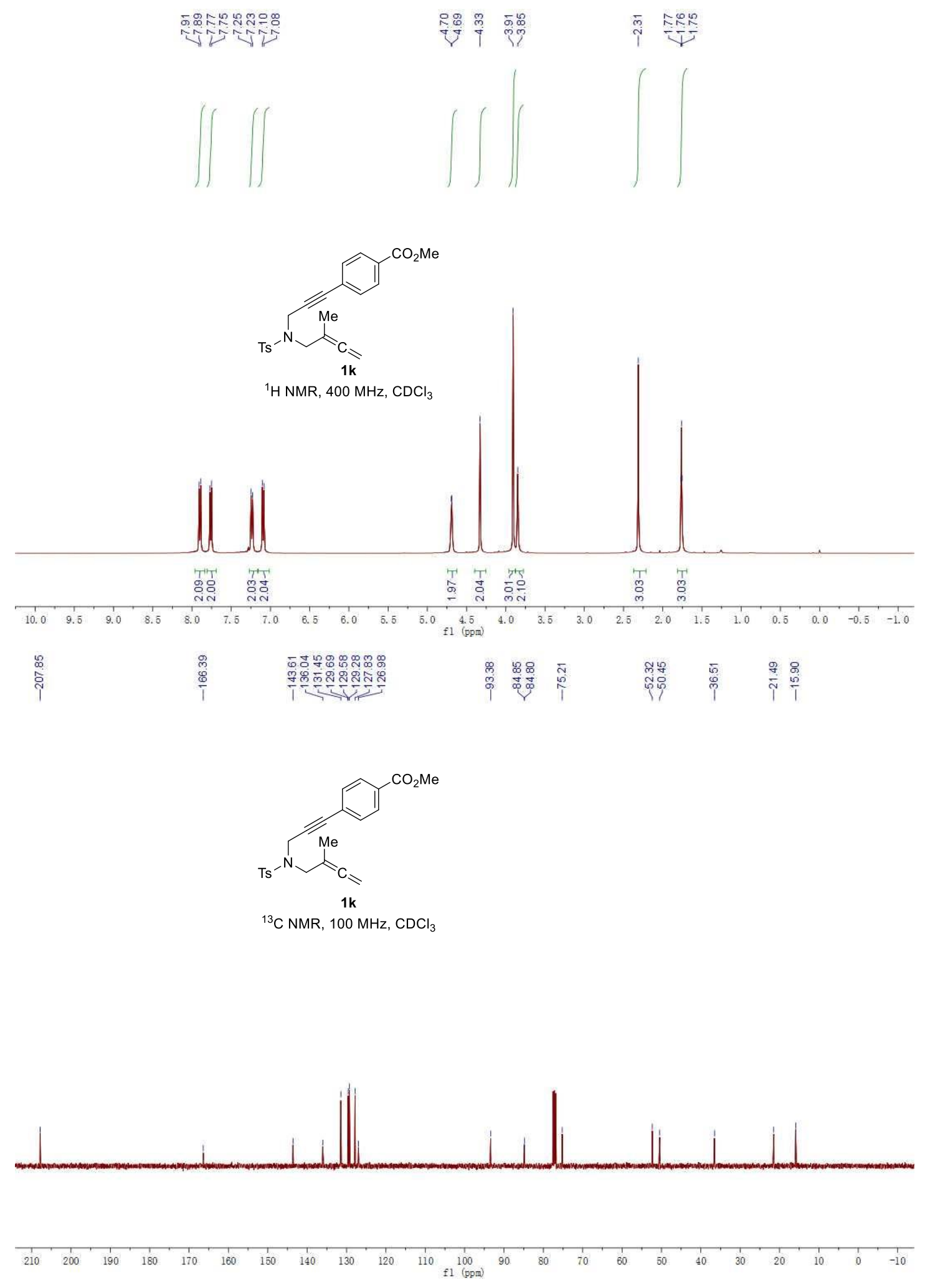



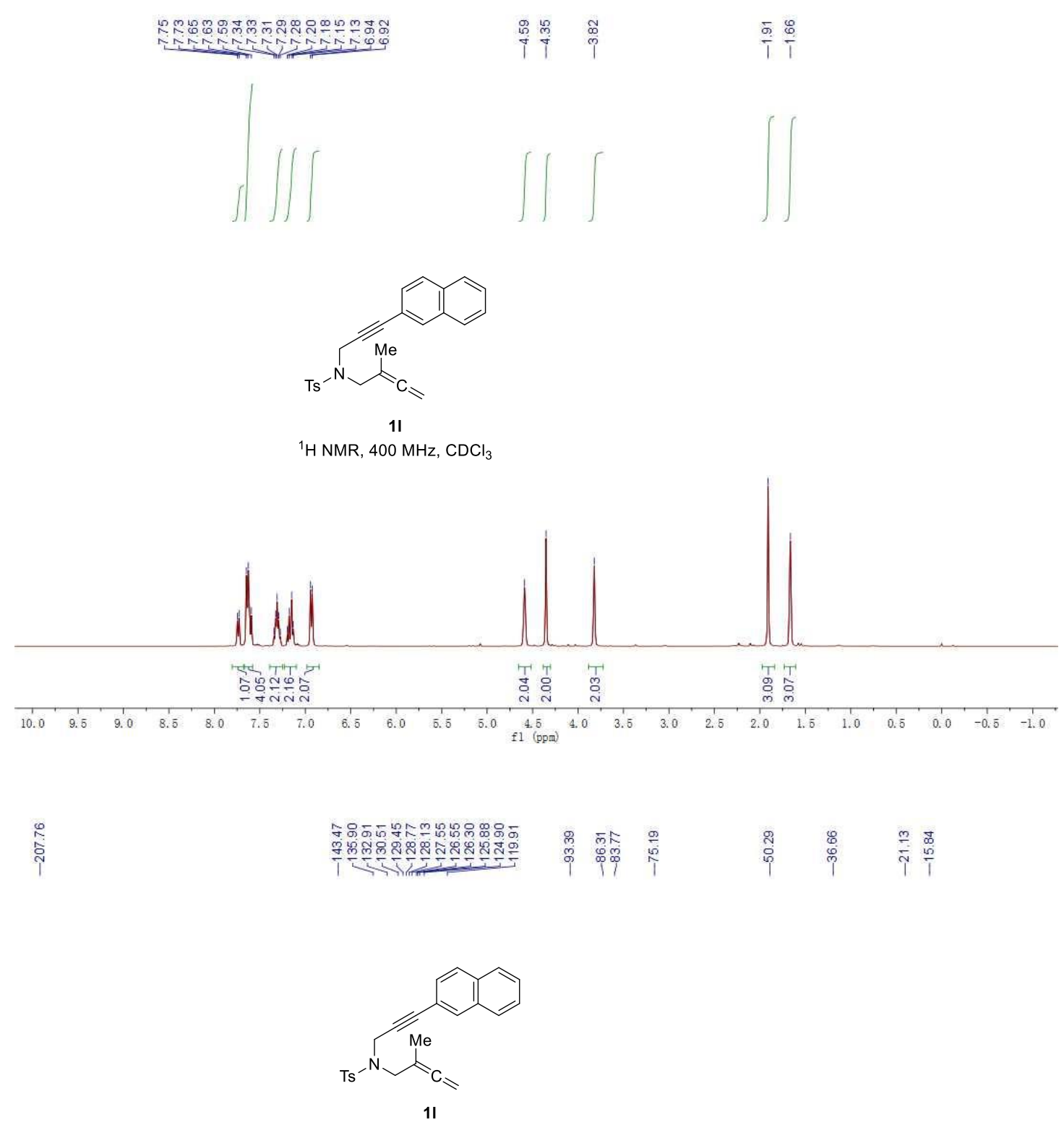

${ }^{13} \mathrm{C} \mathrm{NMR,}, 100 \mathrm{MHz}, \mathrm{CDCl}_{3}$

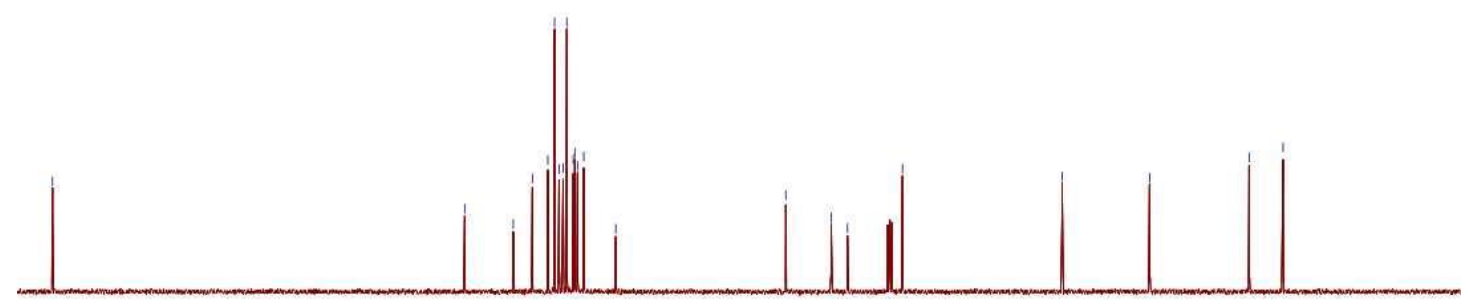

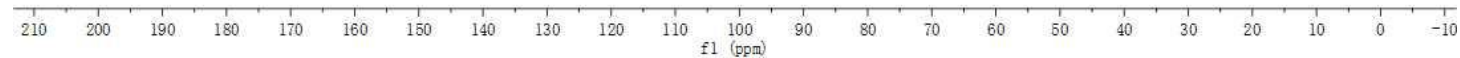




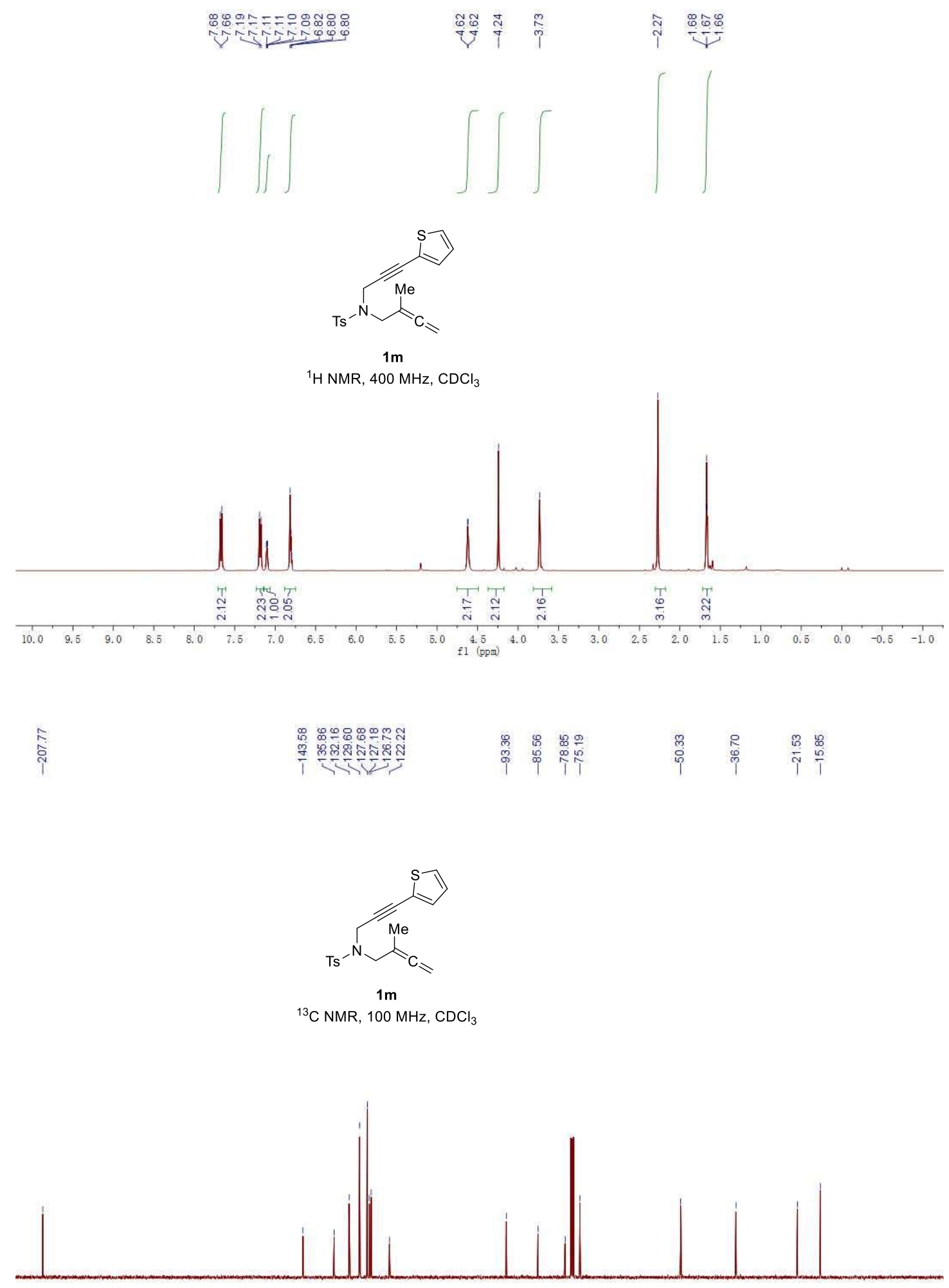

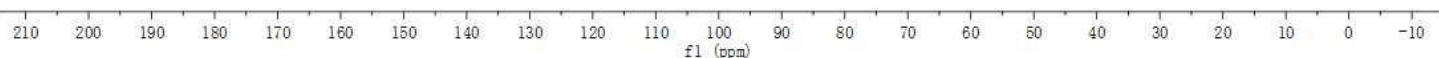




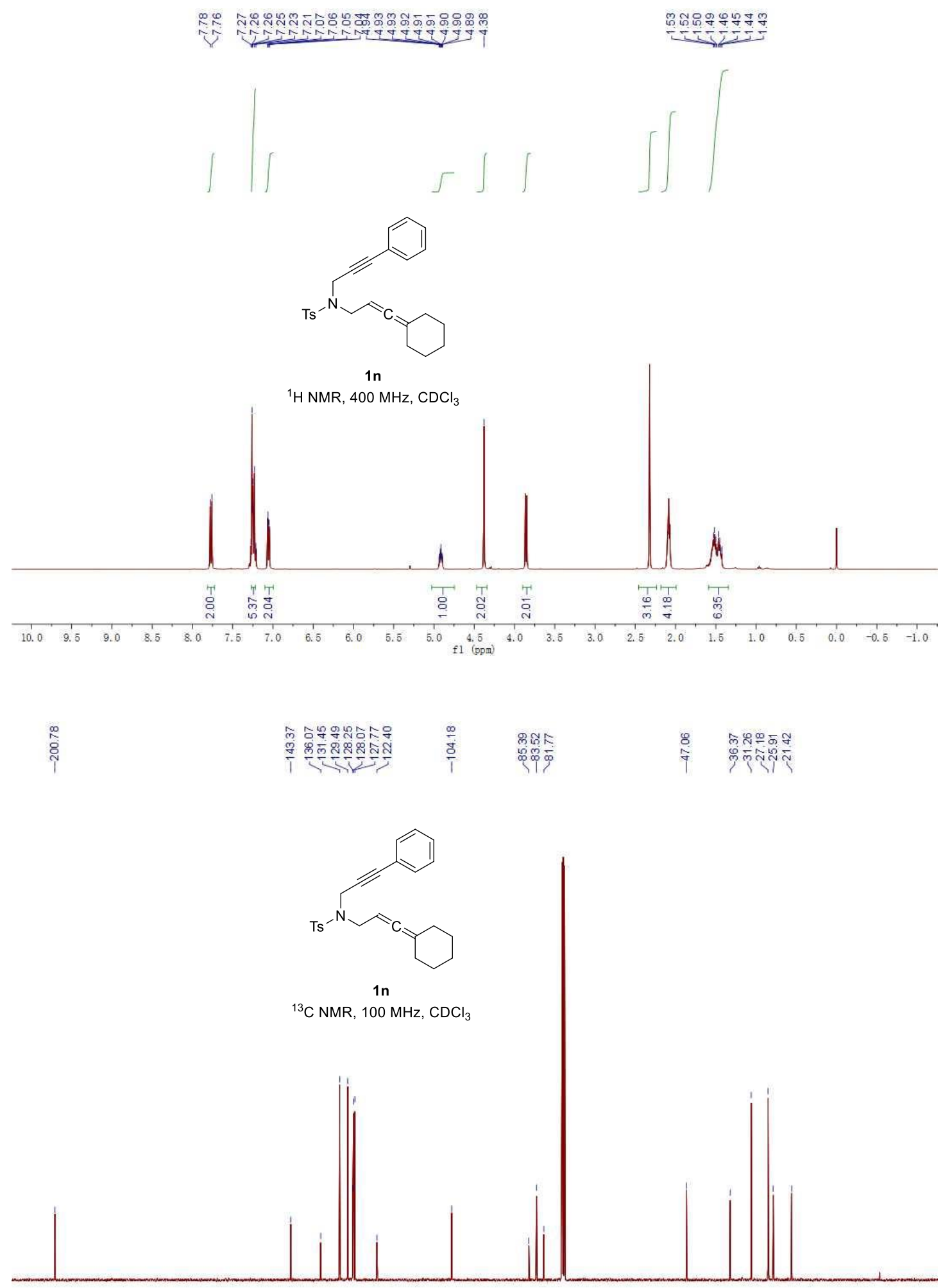

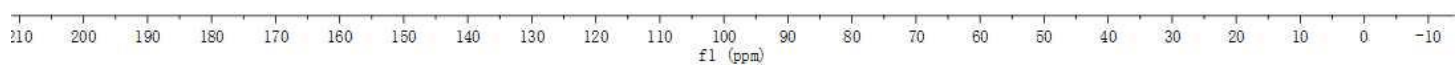




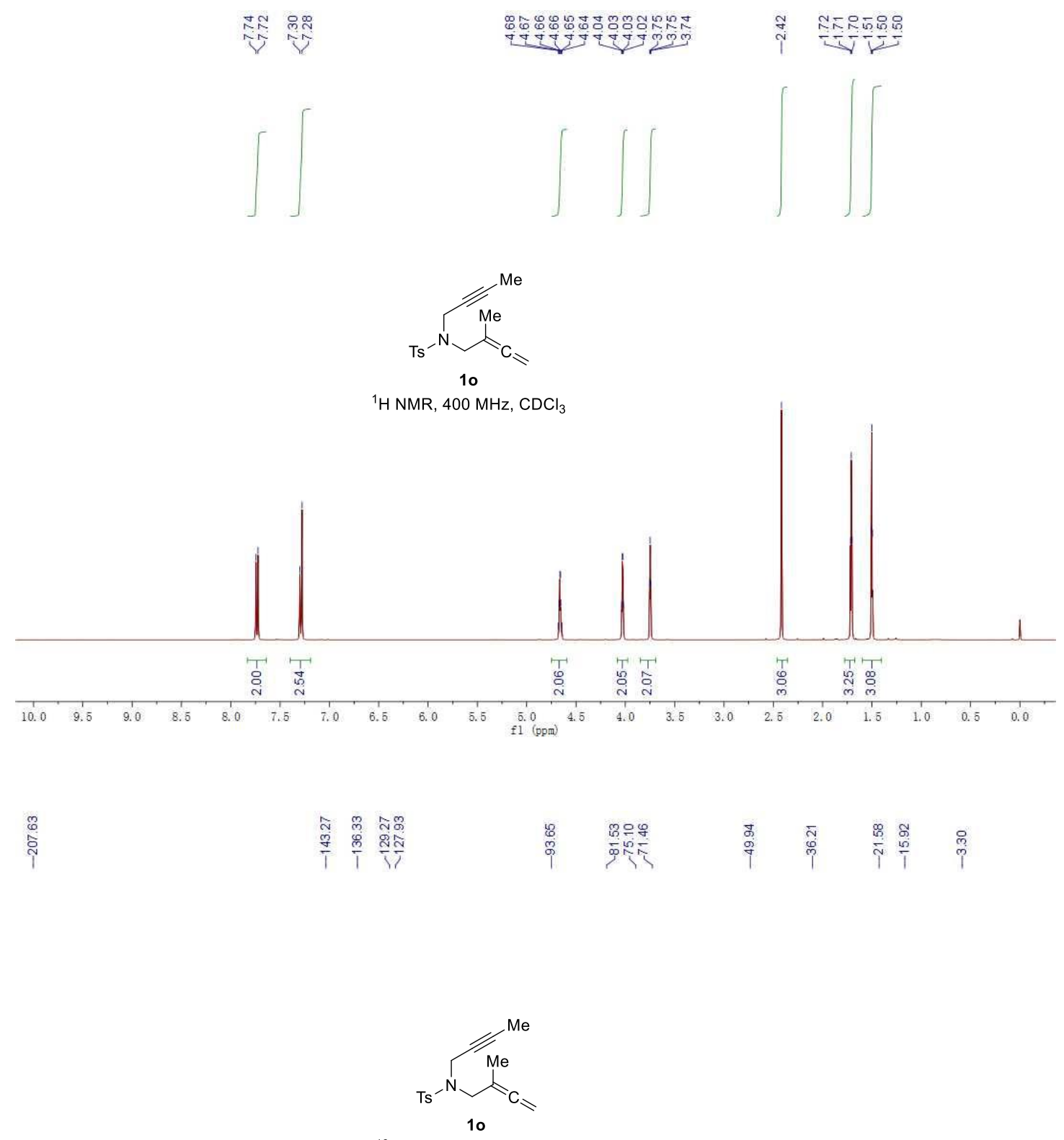

${ }^{13} \mathrm{C} \mathrm{NMR}, 100 \mathrm{MHz}, \mathrm{CDCl}_{3}$

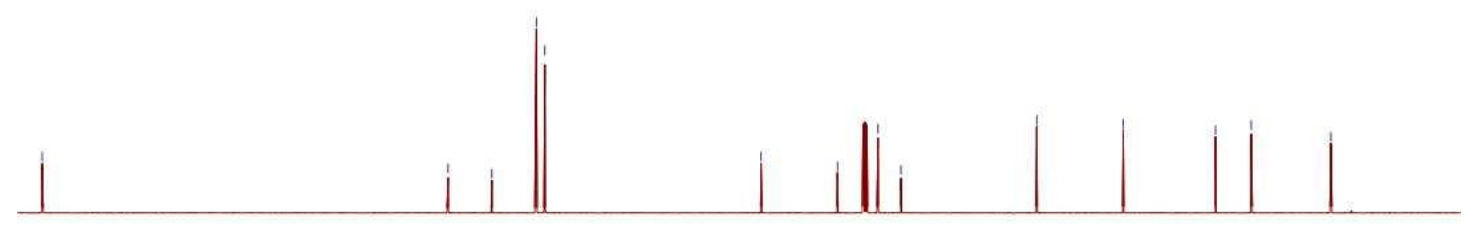

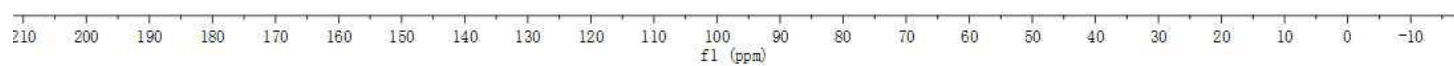




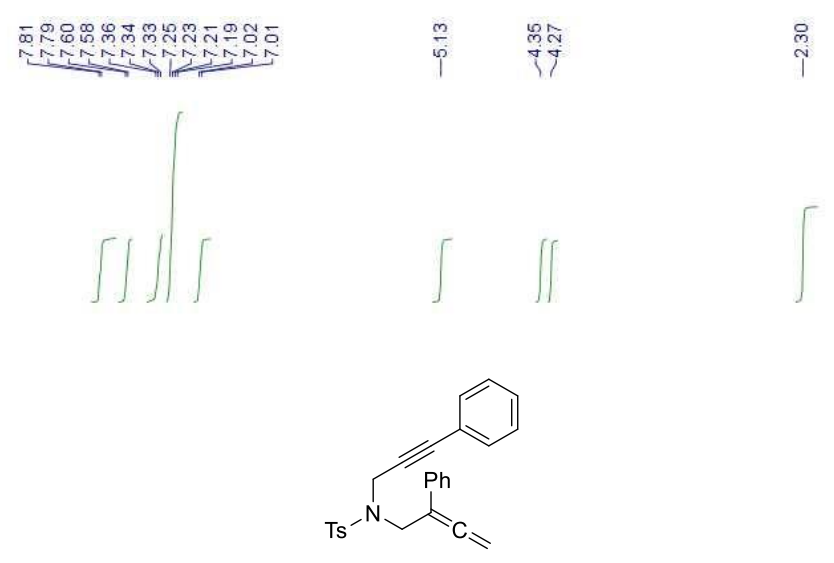

${ }^{1} \mathrm{H} \mathrm{NMR}, 400 \mathrm{MHz}, \mathrm{CDCl}_{3}$
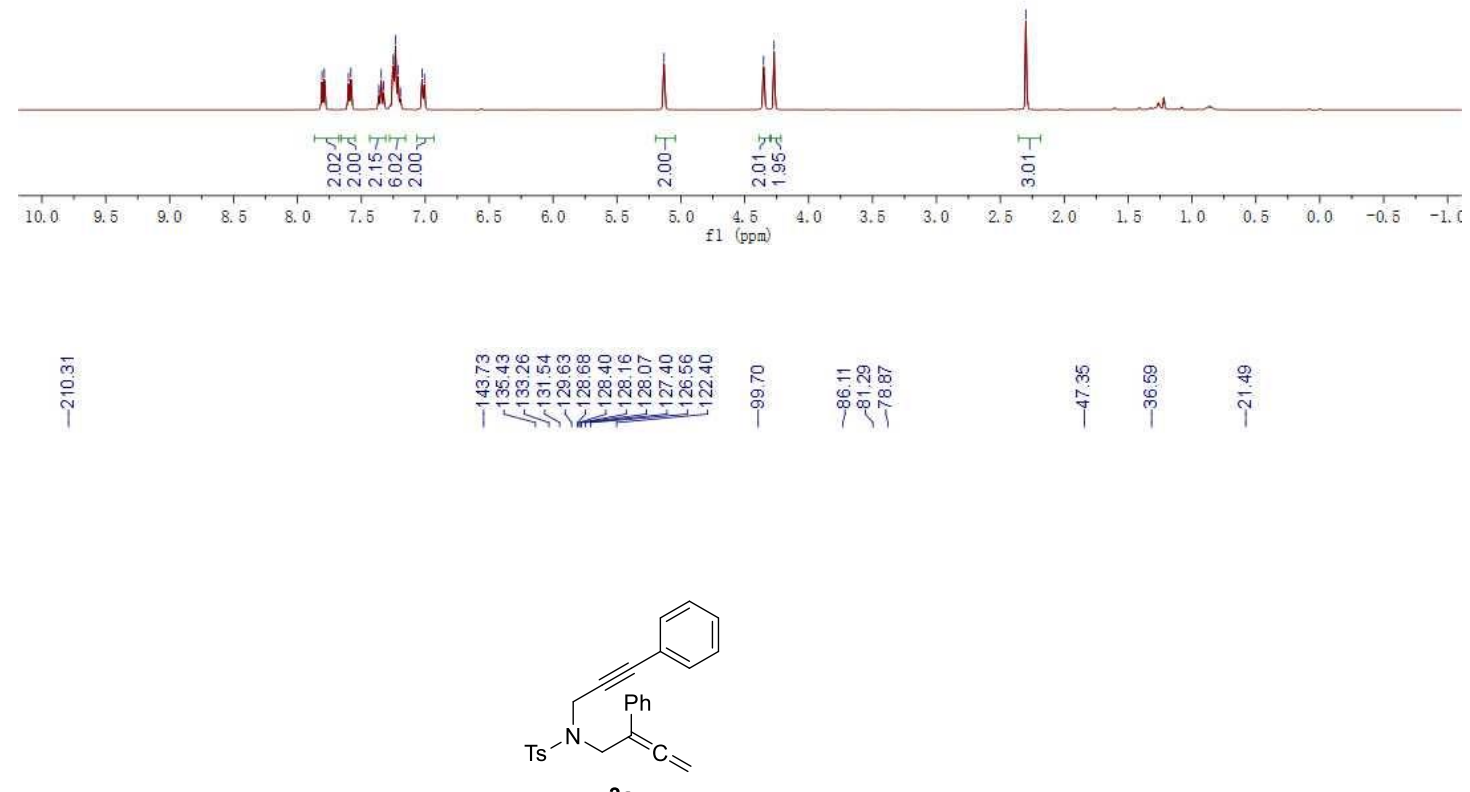

${ }^{13} \mathrm{C} \mathrm{NMR}, 100 \mathrm{MHz}, \mathrm{CDCl}_{3}$

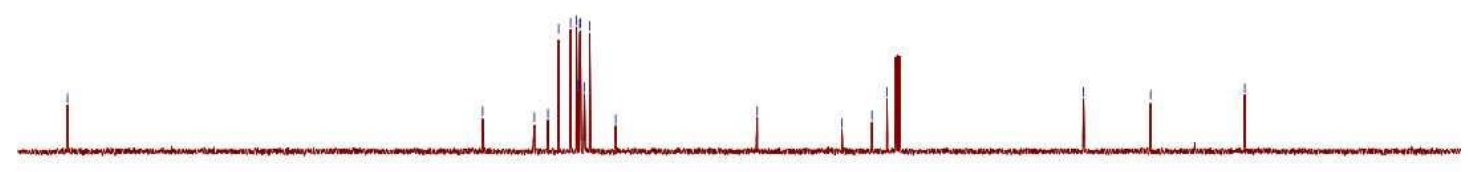

$\begin{array}{llllllllllll}210 & 200 & 190 & 180 & 170 & 160 & 150 & 140 & 130 & 120 & 110 & 100 \\ & & & & & 1\end{array}$ 

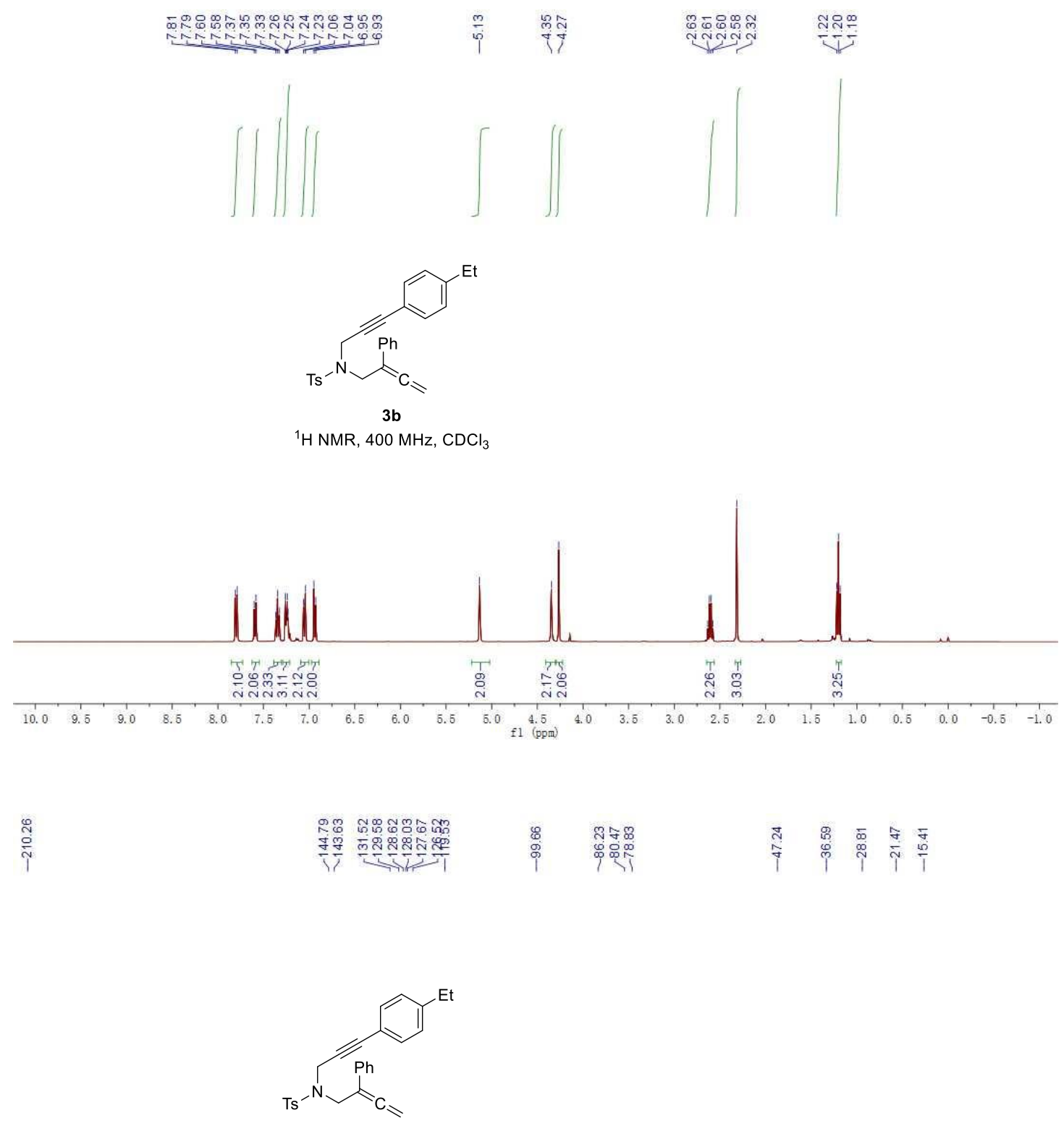

3b

${ }^{13} \mathrm{C} \mathrm{NMR}, 100 \mathrm{MHz}, \mathrm{CDCl}_{3}$

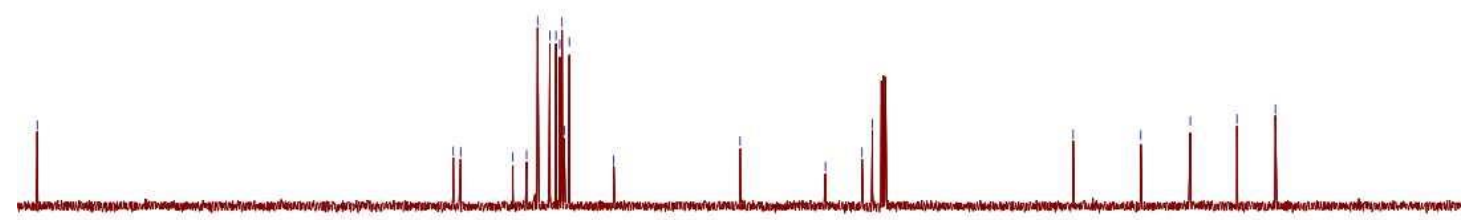

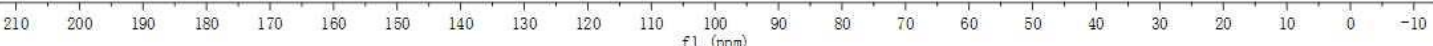



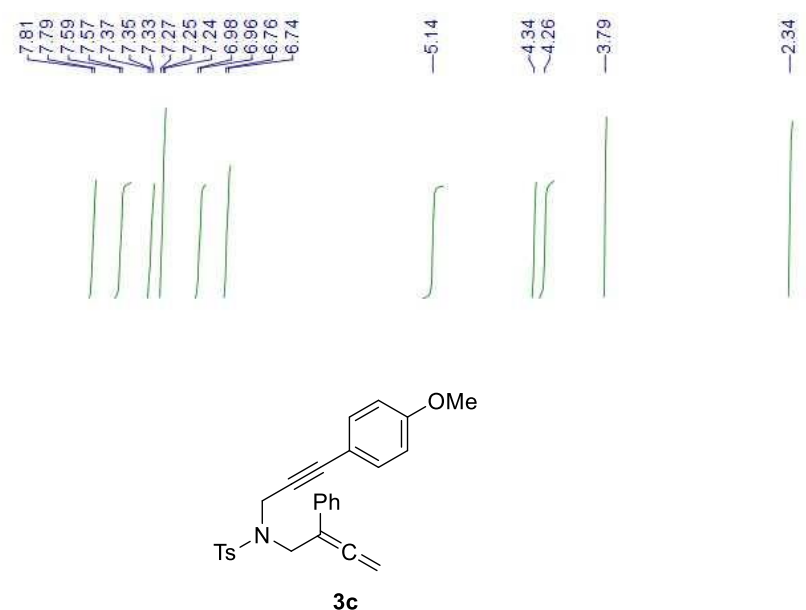

${ }^{1} \mathrm{H}$ NMR, $400 \mathrm{MHz}, \mathrm{CDCl}_{3}$

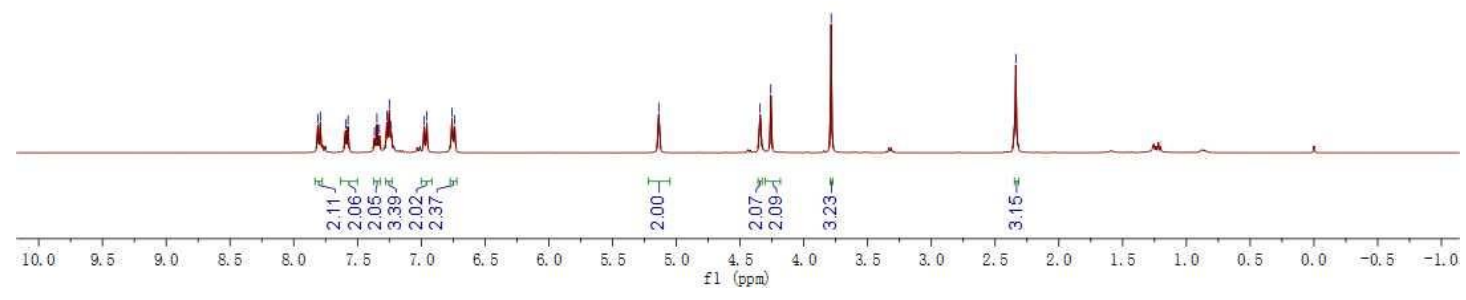

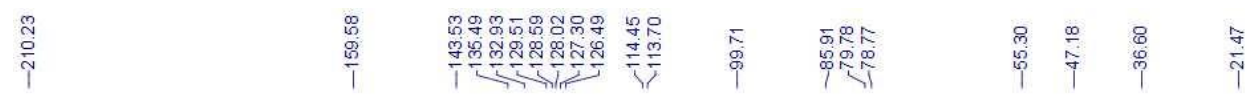

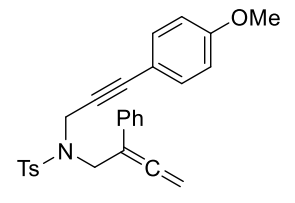

$3 c$

${ }^{13} \mathrm{C} \mathrm{NMR}, 100 \mathrm{MHz}, \mathrm{CDCl}_{3}$

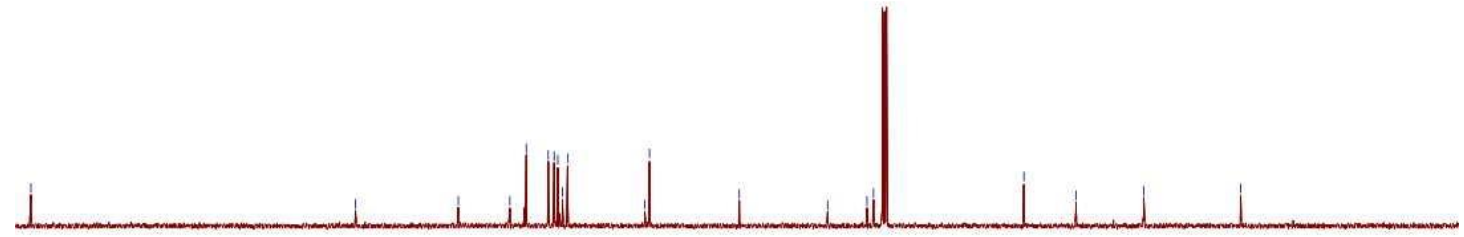

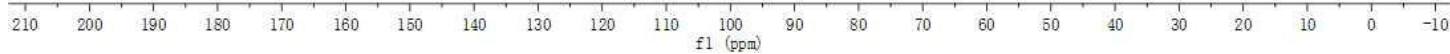




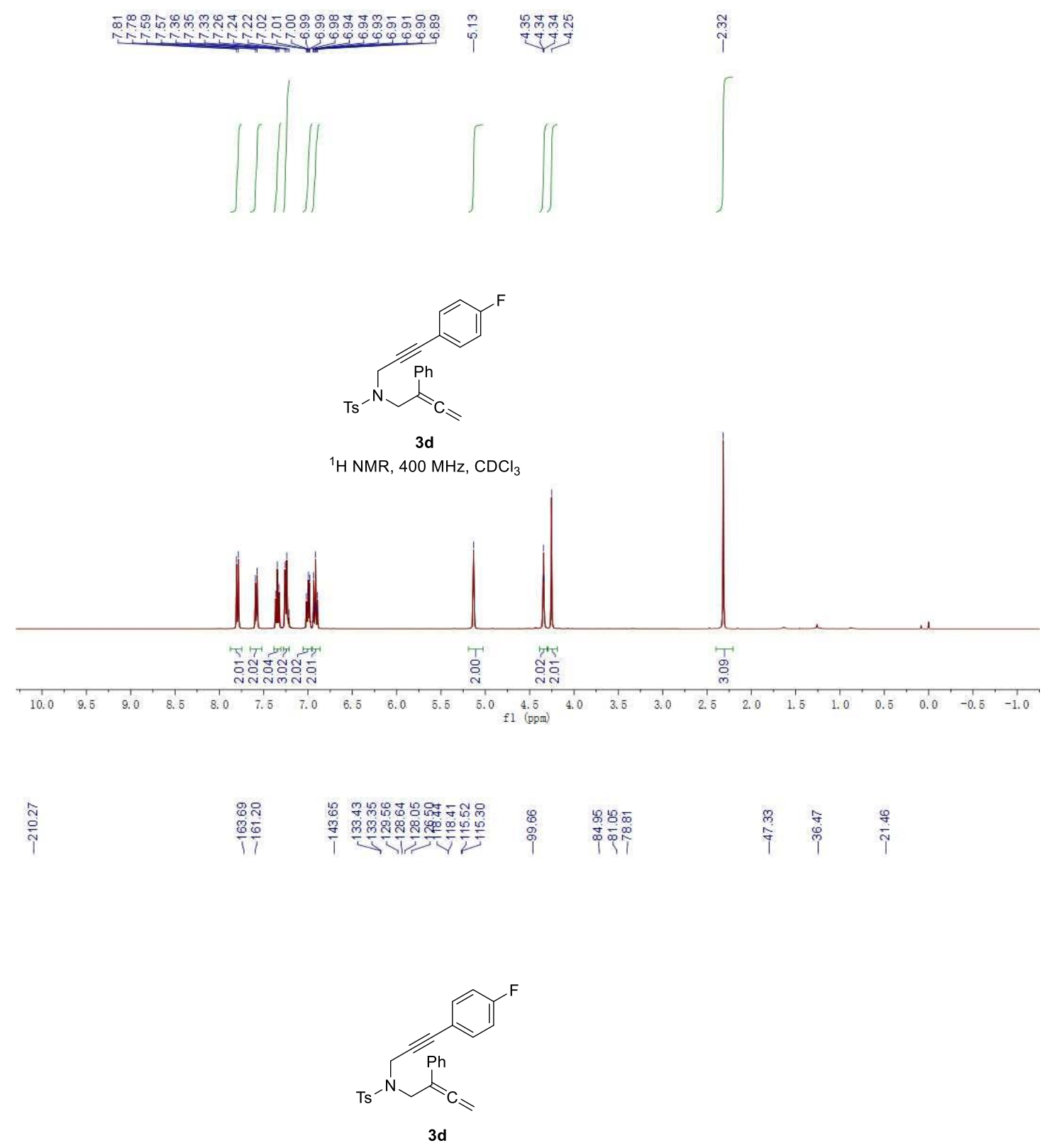

${ }^{13} \mathrm{C} \mathrm{NMR}, 100 \mathrm{MHz}, \mathrm{CDCl}_{3}$

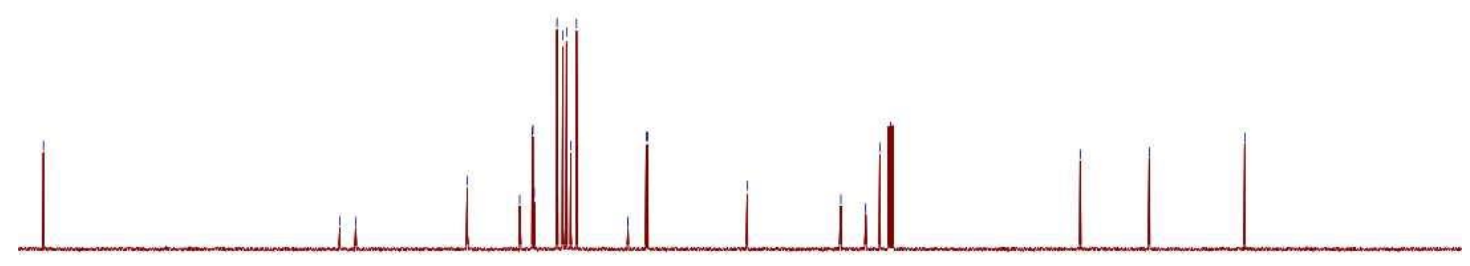

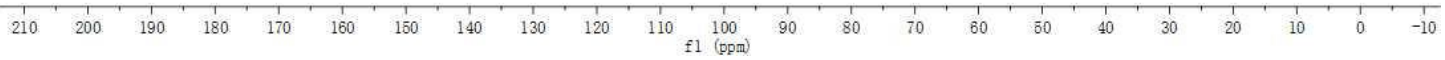



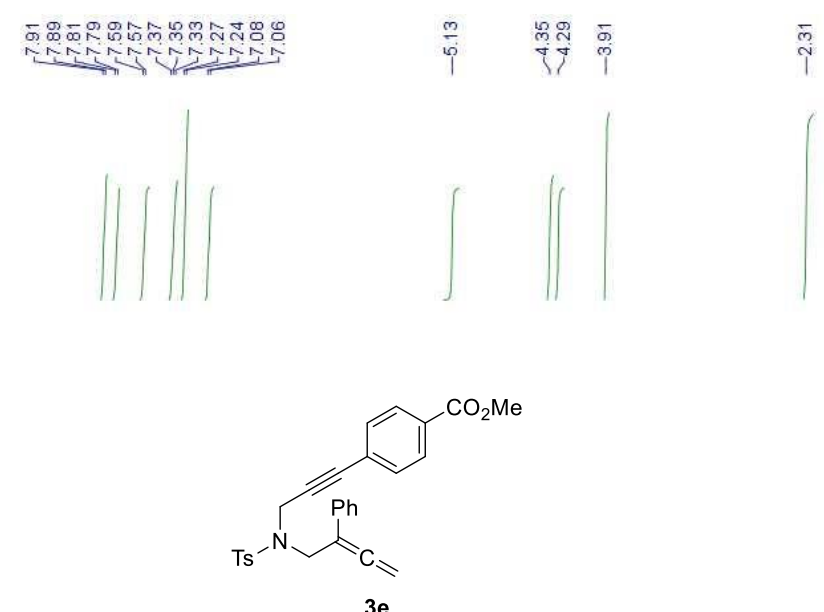

${ }^{1} \mathrm{H} \mathrm{NMR}, 400 \mathrm{MHz}, \mathrm{CDCl}_{3}$

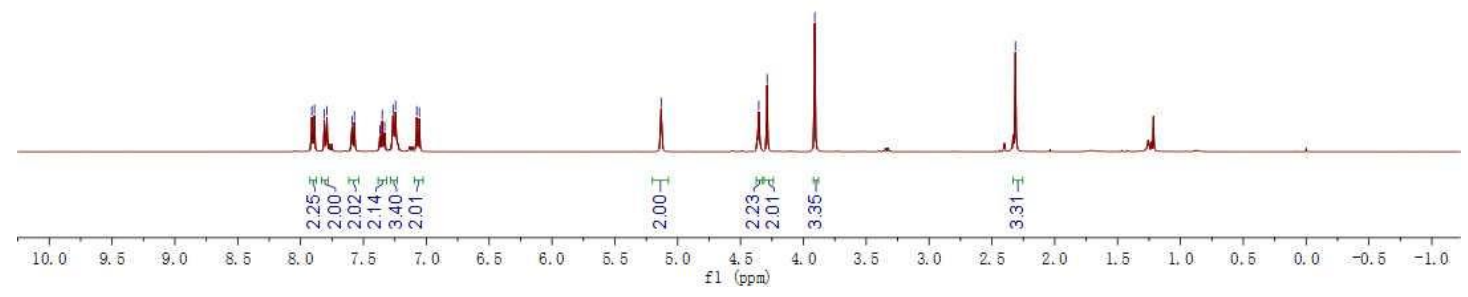

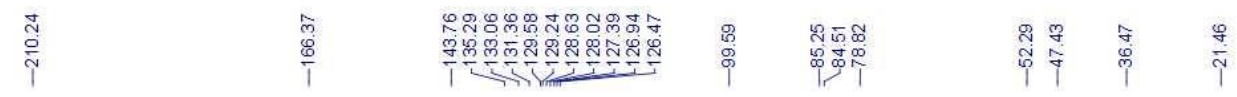

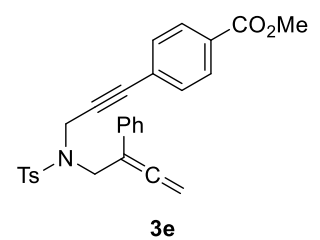

${ }^{13} \mathrm{C} \mathrm{NMR}, 100 \mathrm{MHz}, \mathrm{CDCl}_{3}$

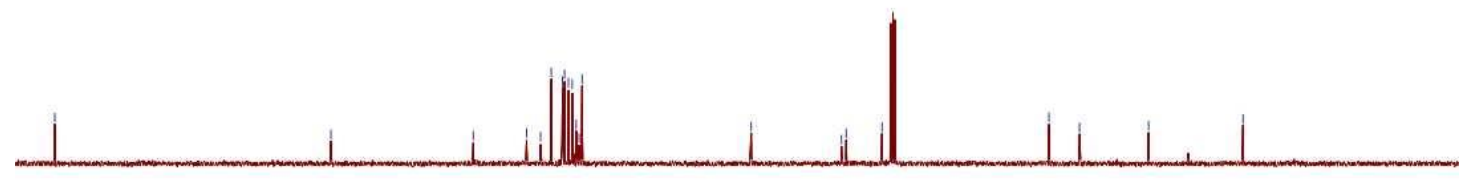

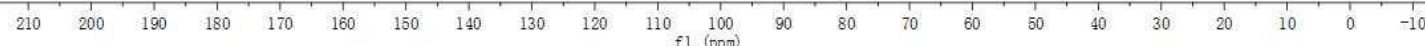




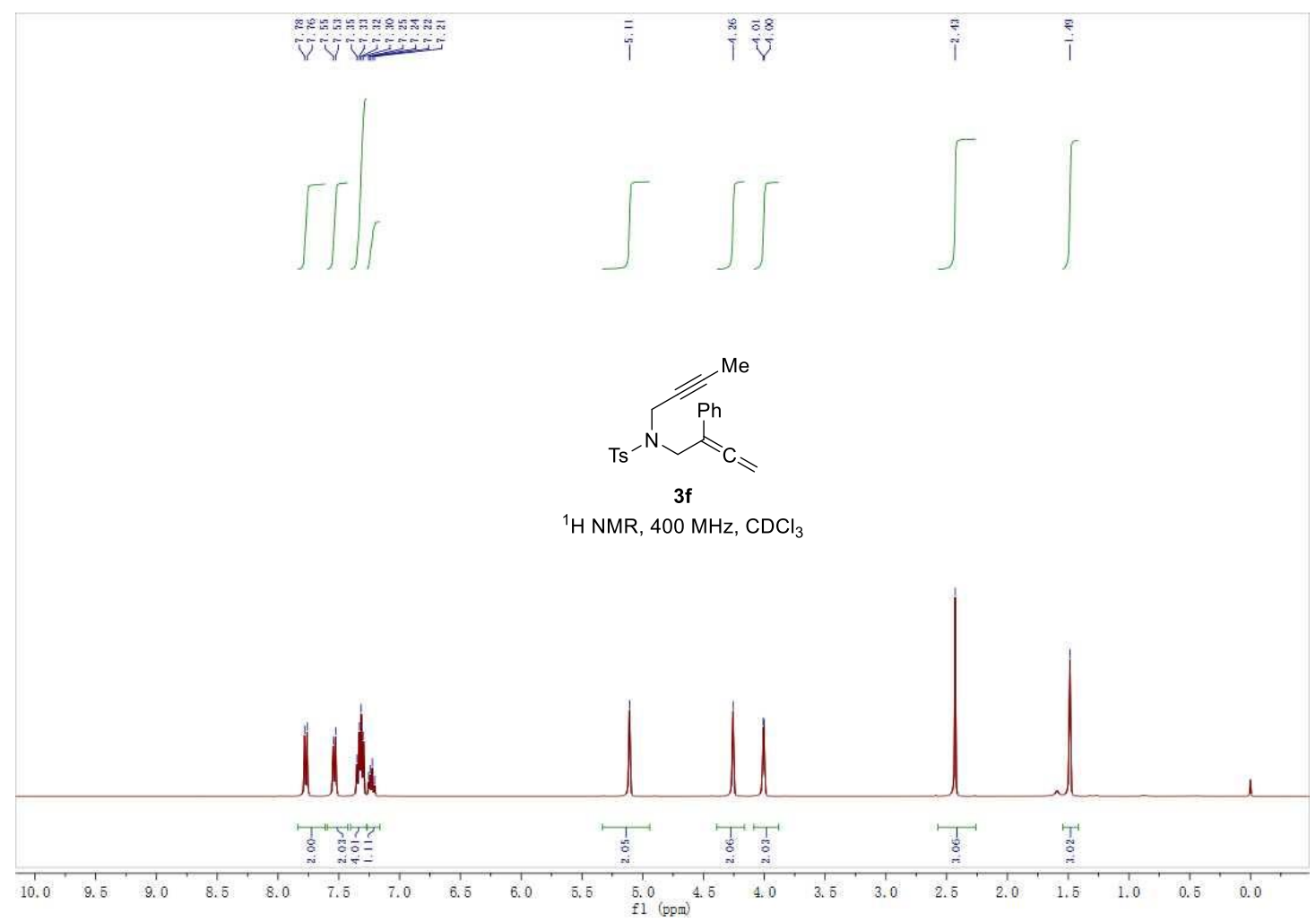

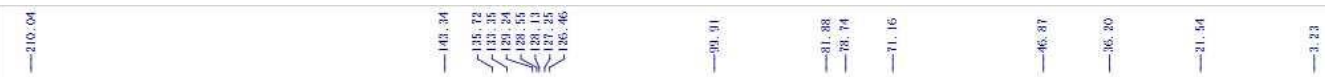
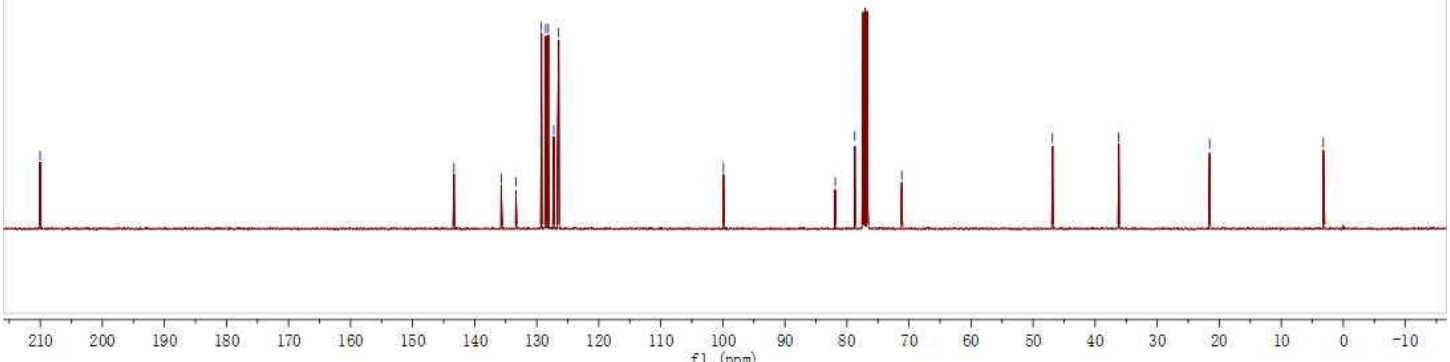


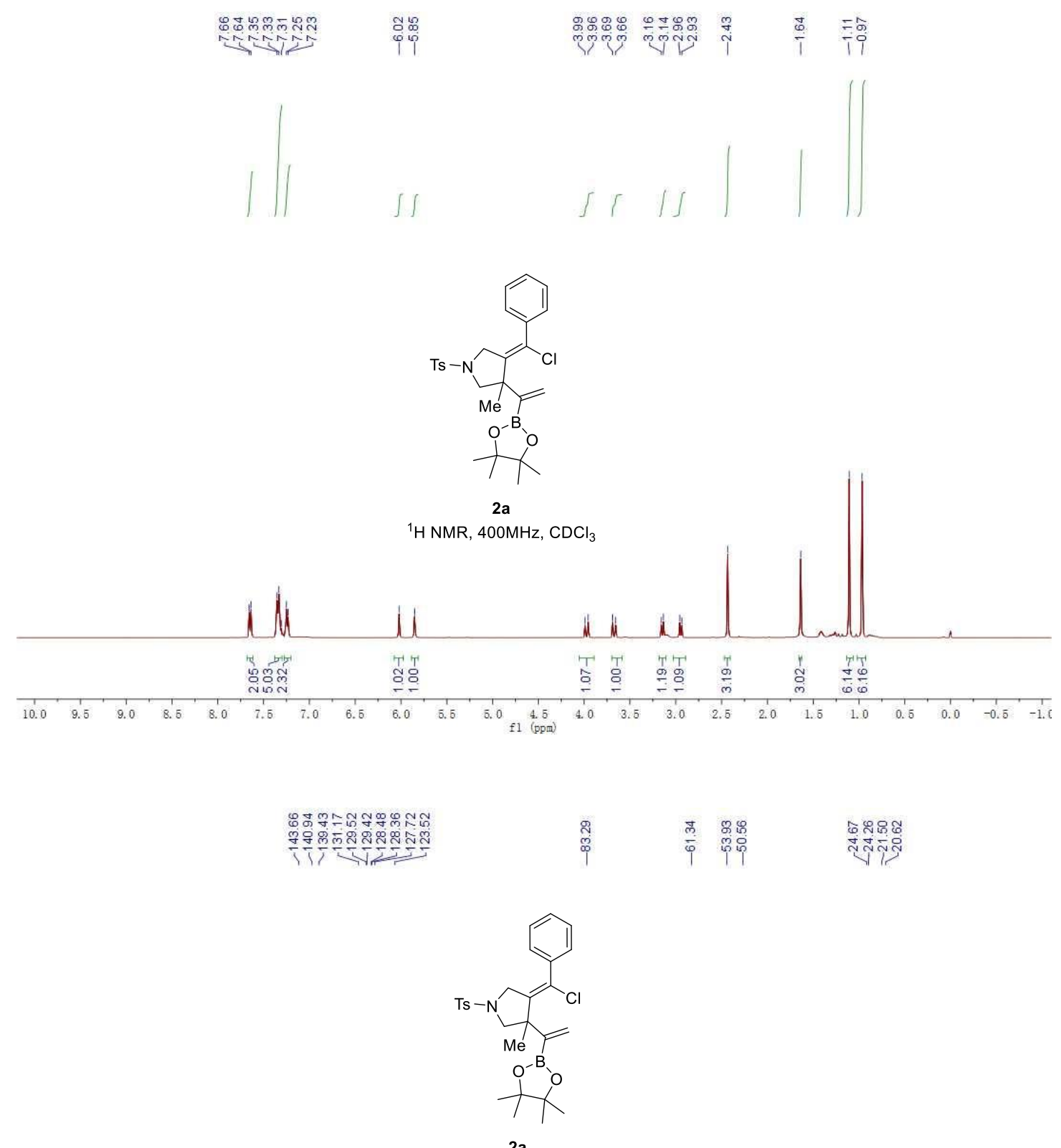

${ }^{13} \mathrm{C} \mathrm{NMR}, 100 \mathrm{MHz}, \mathrm{CDCl}_{3}$

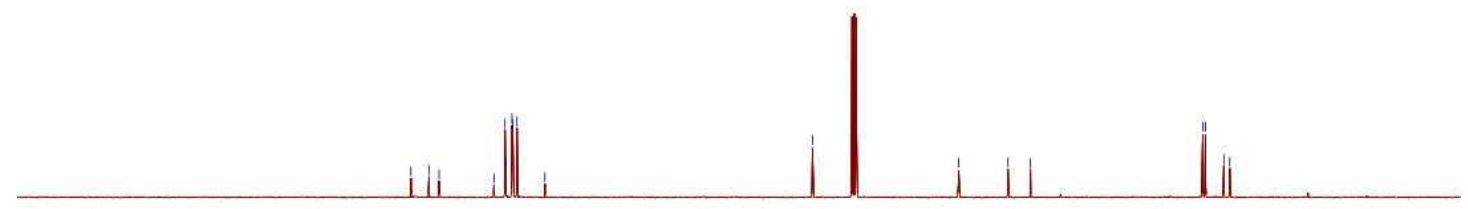

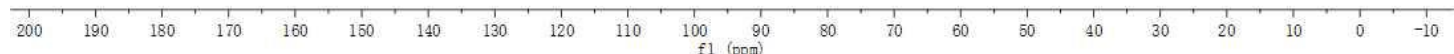




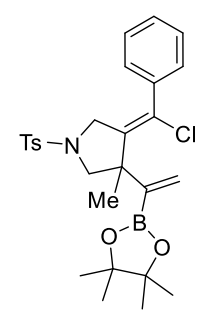

2a

${ }^{11} \mathrm{~B} N M R, 128 \mathrm{MHz}, \mathrm{CDCl}_{3}$

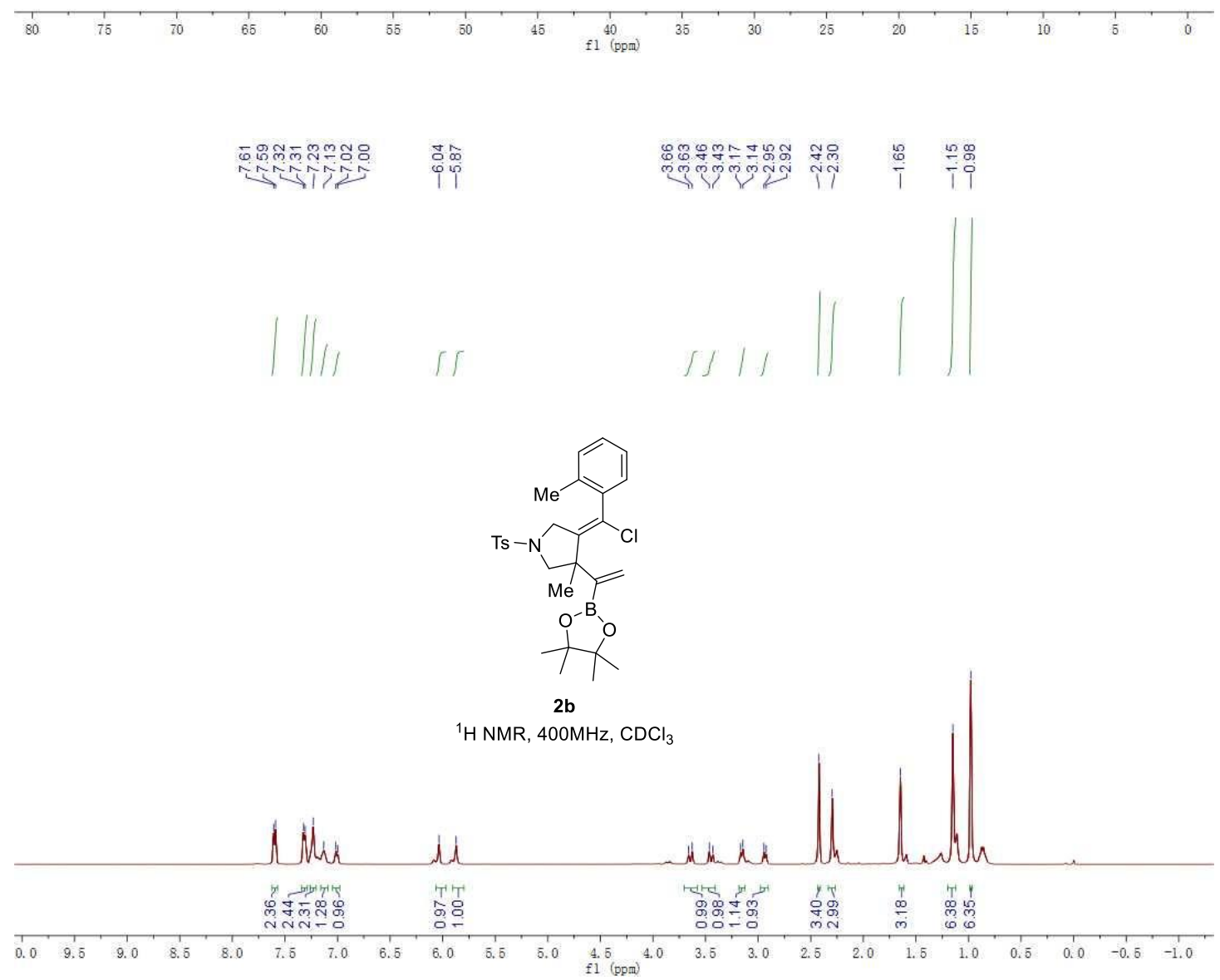




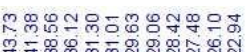

学近以

年

ㄱำ

ㄴ..ㄴ. - T

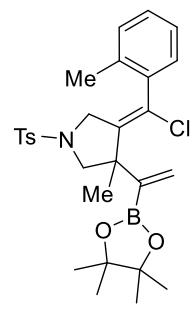

${ }^{13} \mathrm{C} \mathrm{NMR}, 100 \mathrm{MHz}, \mathrm{CDCl}_{3}$
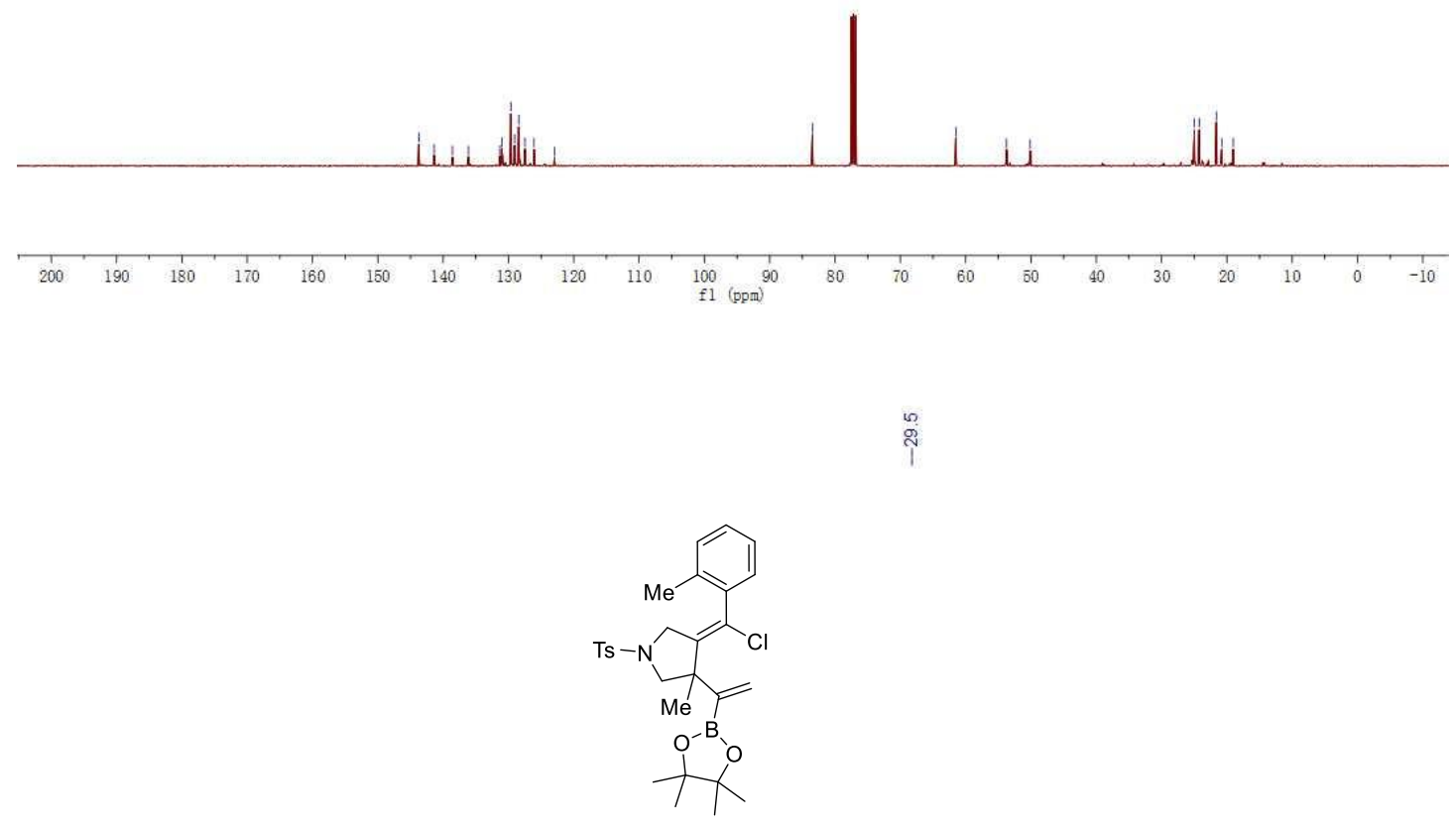

2b

${ }^{11} \mathrm{~B}$ NMR, $128 \mathrm{MHz}, \mathrm{CDCl}_{3}$

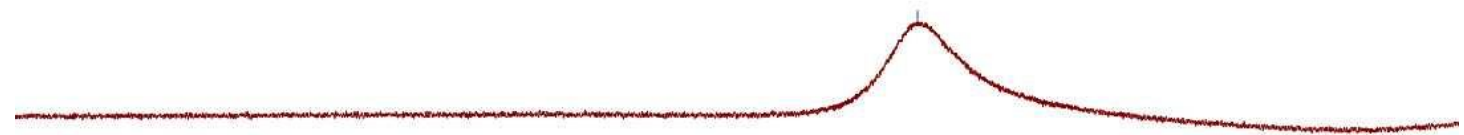

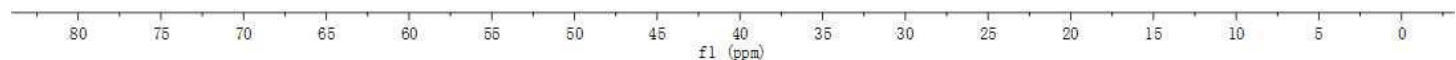




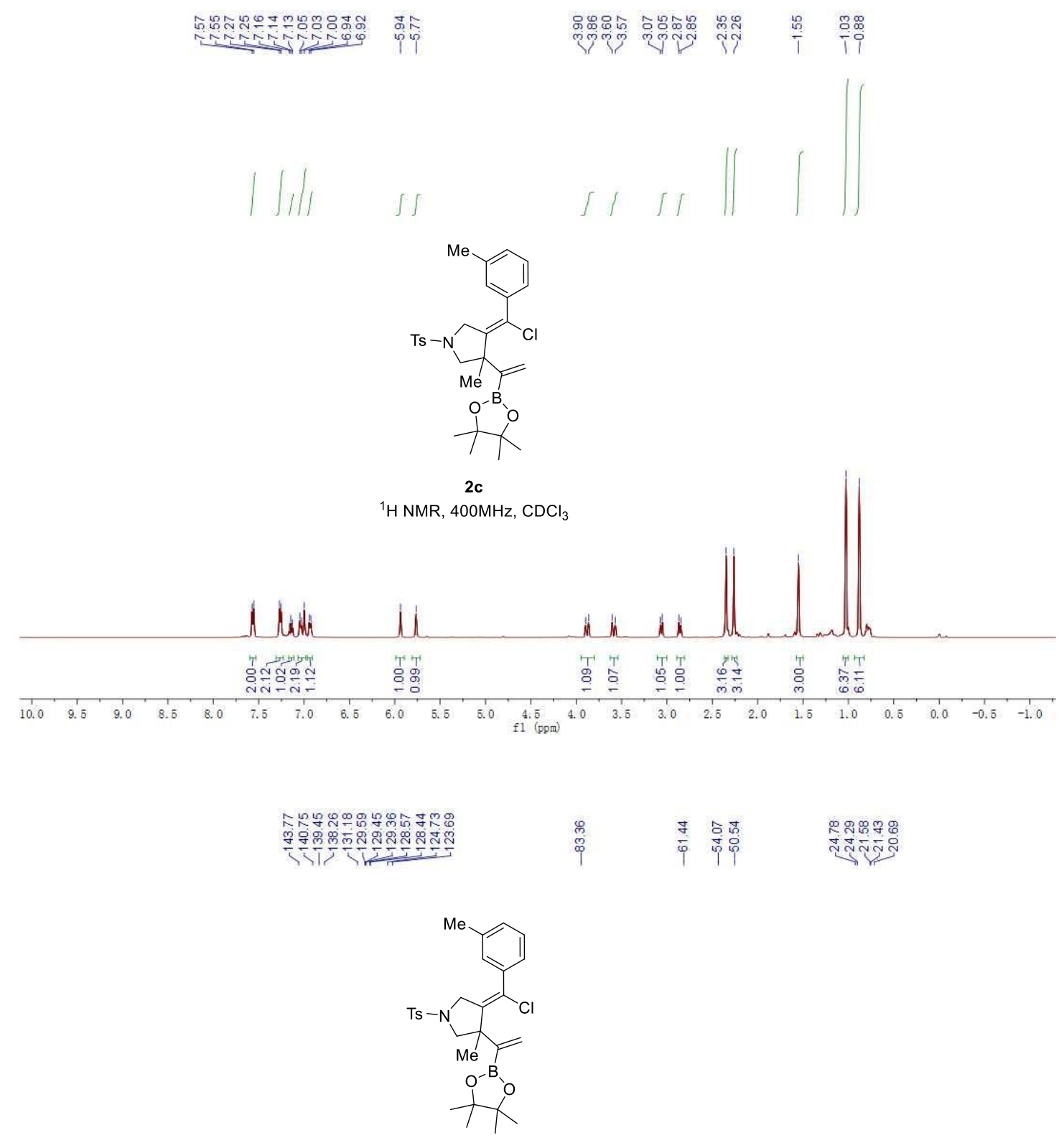

${ }^{13} \mathrm{C} \mathrm{NMR,}, 100 \mathrm{MHz}, \mathrm{CDCl}_{3}$

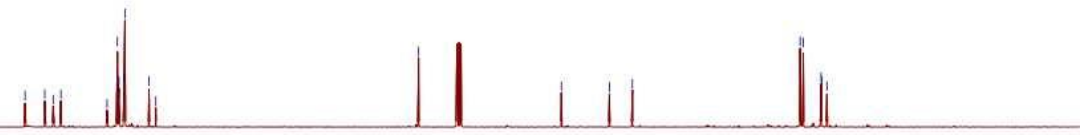

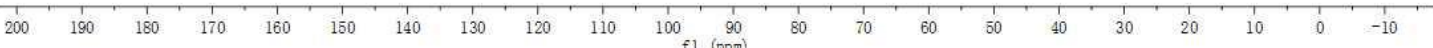




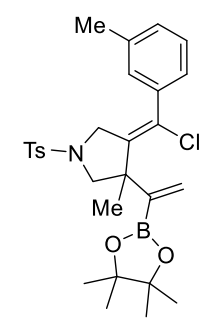

2c

${ }^{11} \mathrm{~B}$ NMR, $128 \mathrm{MHz}, \mathrm{CDCl}_{3}$
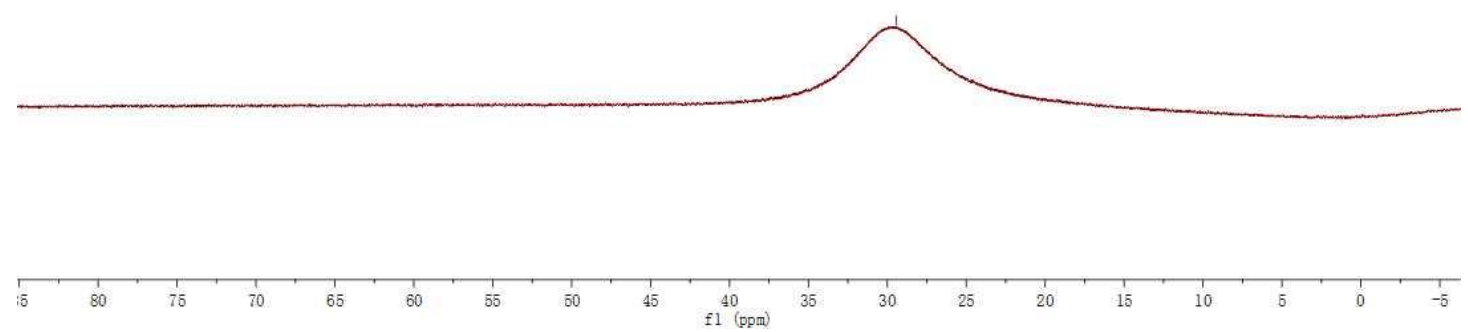

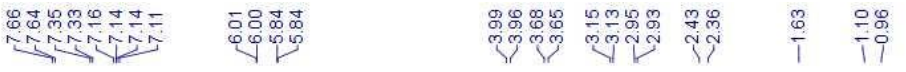

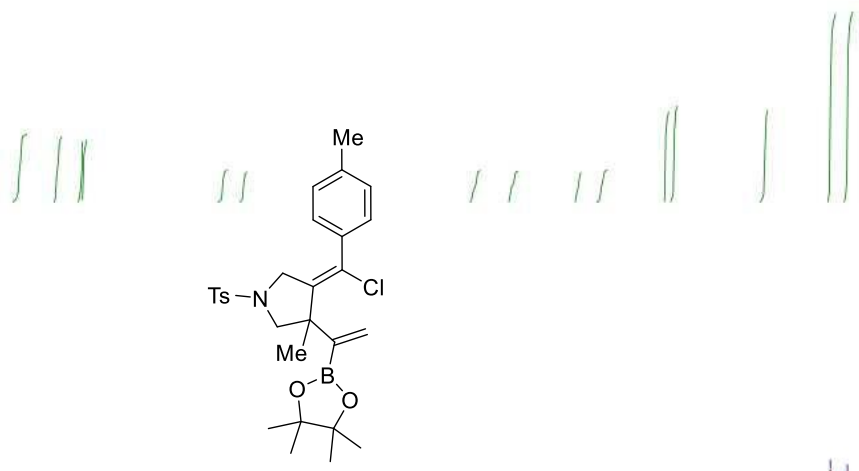

2d

${ }^{1} \mathrm{H}$ NMR, $400 \mathrm{MHz}, \mathrm{CDCl}_{3}$

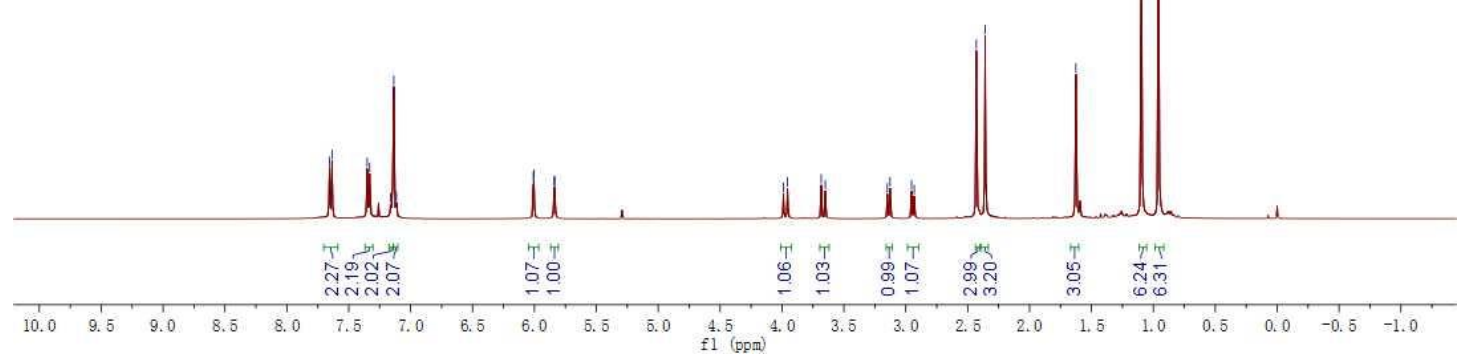



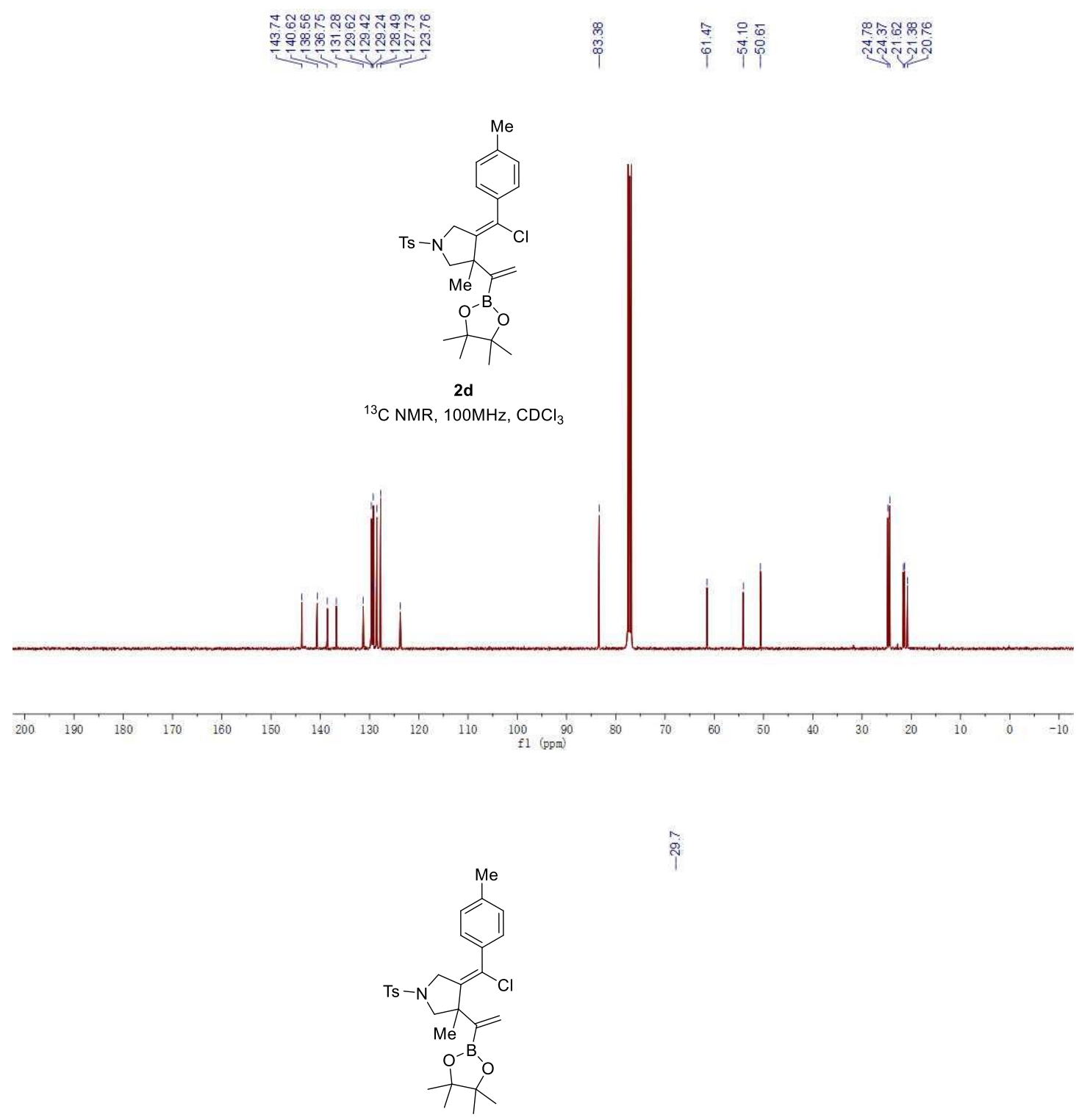

${ }^{11} \mathrm{~B} N M R, 128 \mathrm{MHz}, \mathrm{CDCl}_{3}$

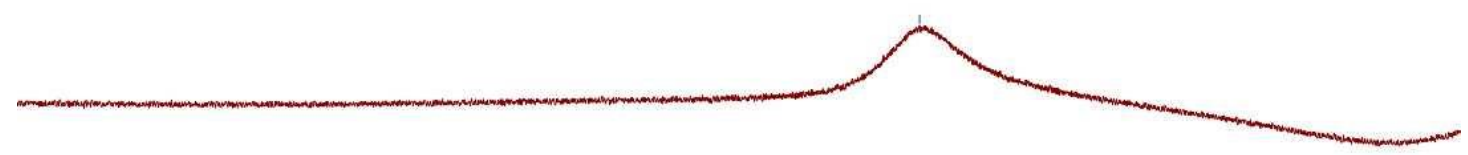

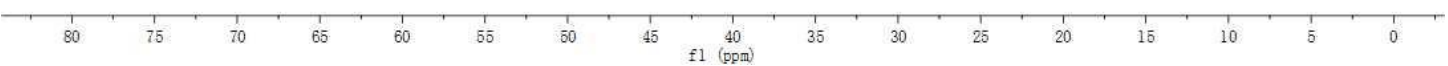




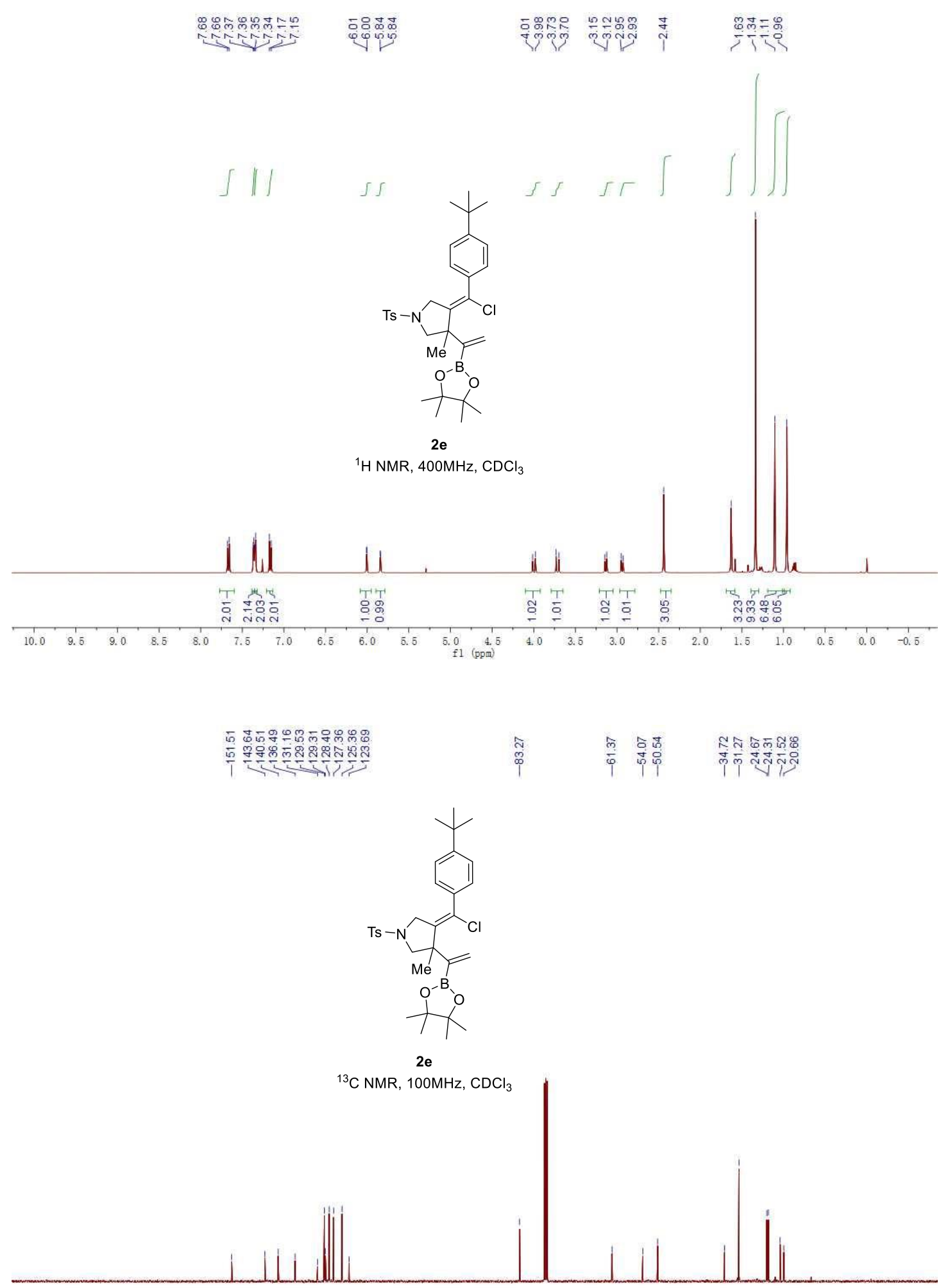

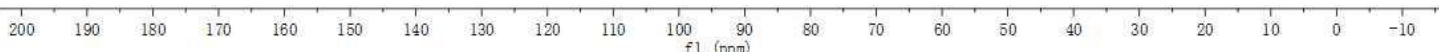




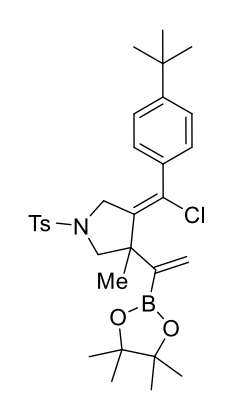

${ }^{11} \mathrm{~B}$ NMR, $128 \mathrm{MHz}, \mathrm{CDCl}_{3}$
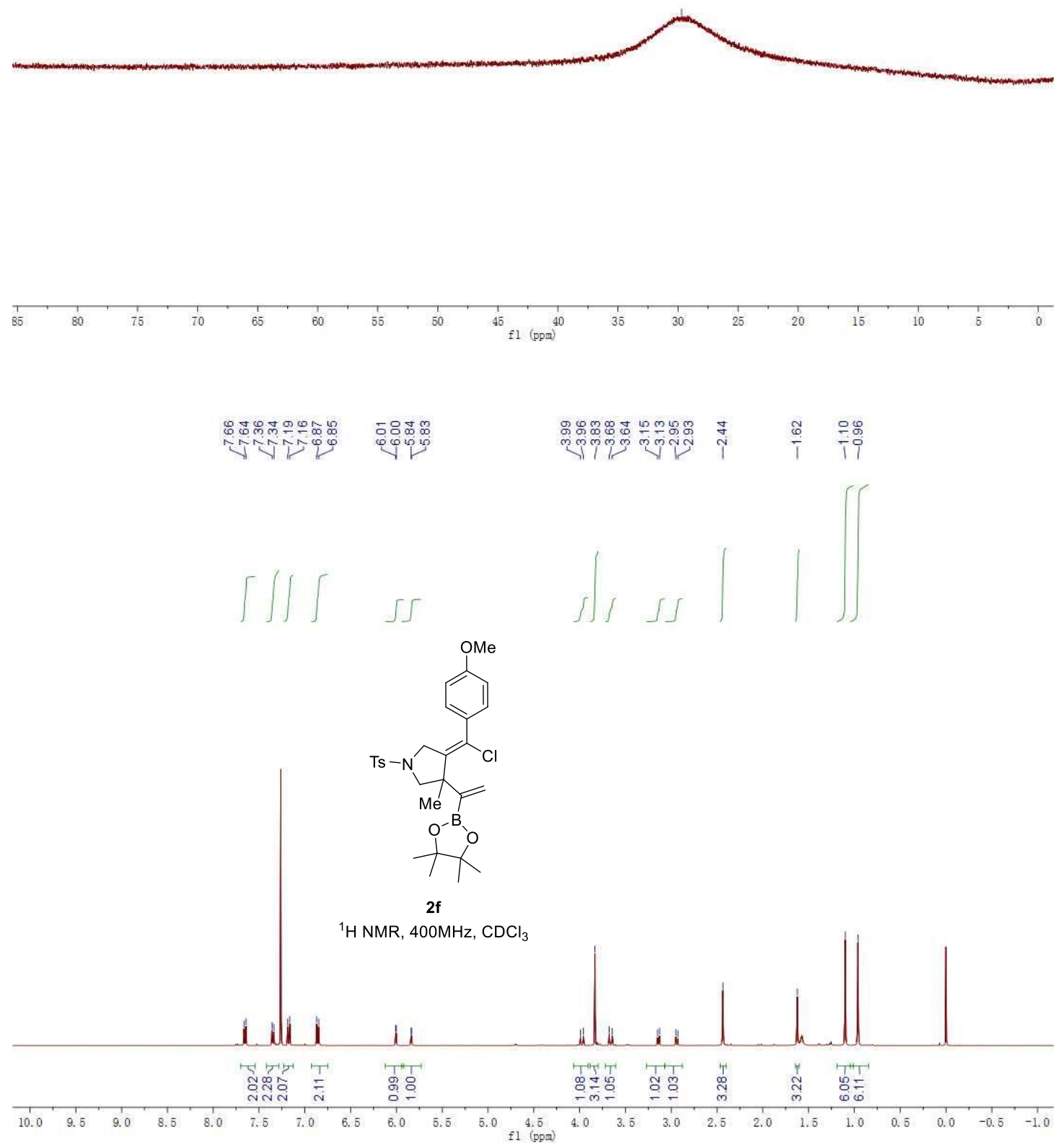

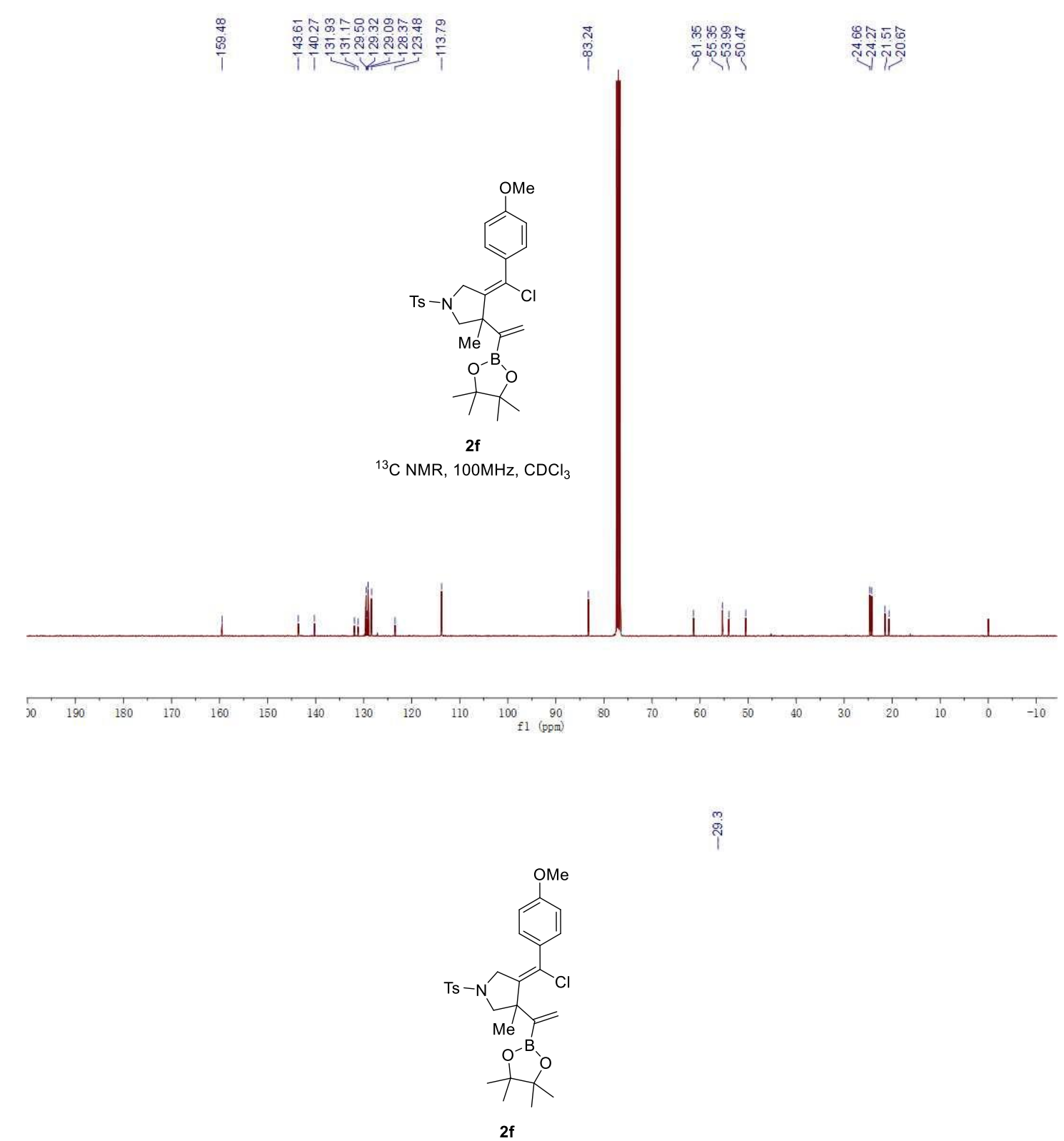

${ }^{11} \mathrm{~B} N M R, 128 \mathrm{MHz}, \mathrm{CDCl}_{3}$

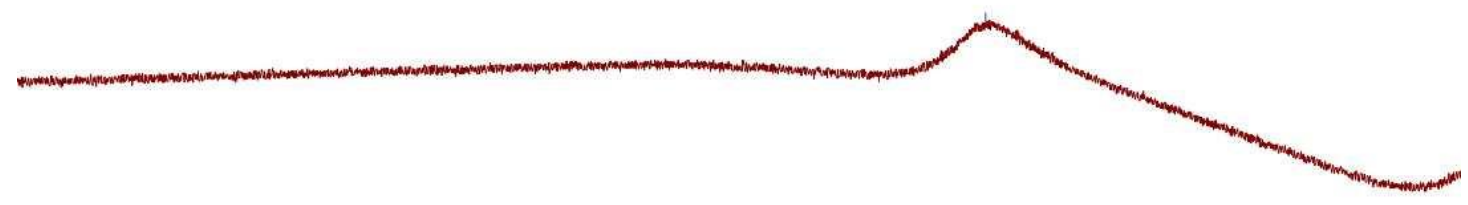

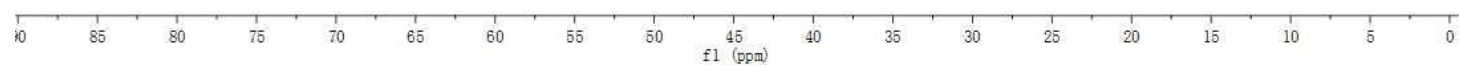




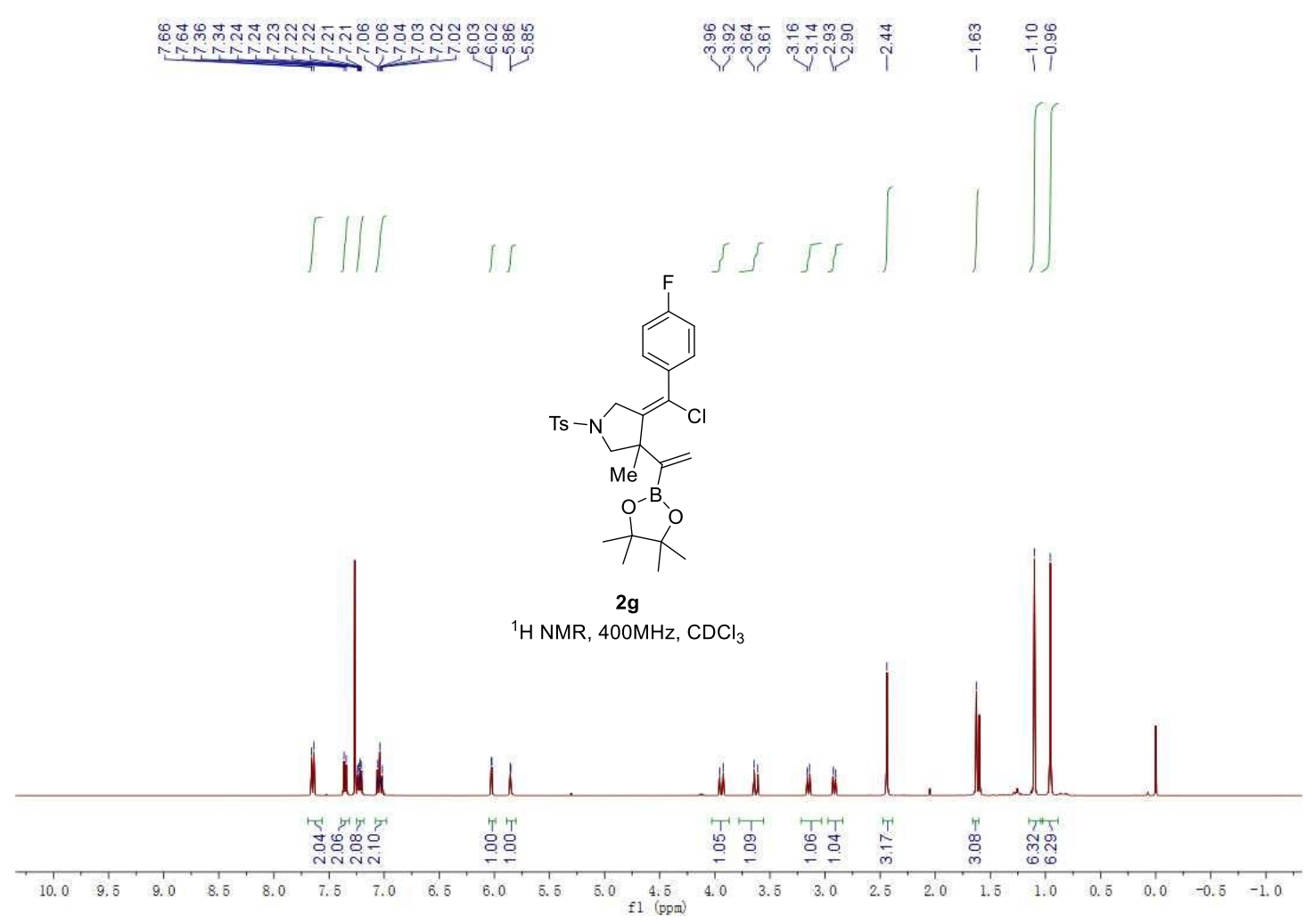

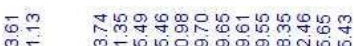

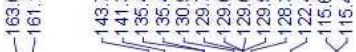

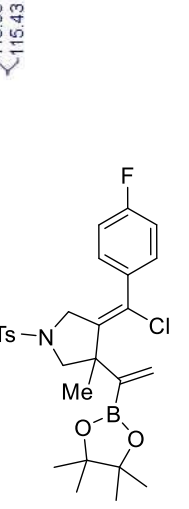

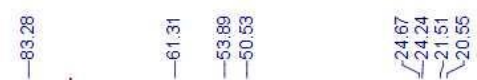

2g

${ }^{13} \mathrm{C} \mathrm{NMR,}, 100 \mathrm{MHz}, \mathrm{CDCl}_{3}$

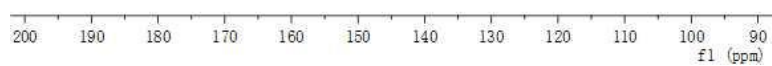
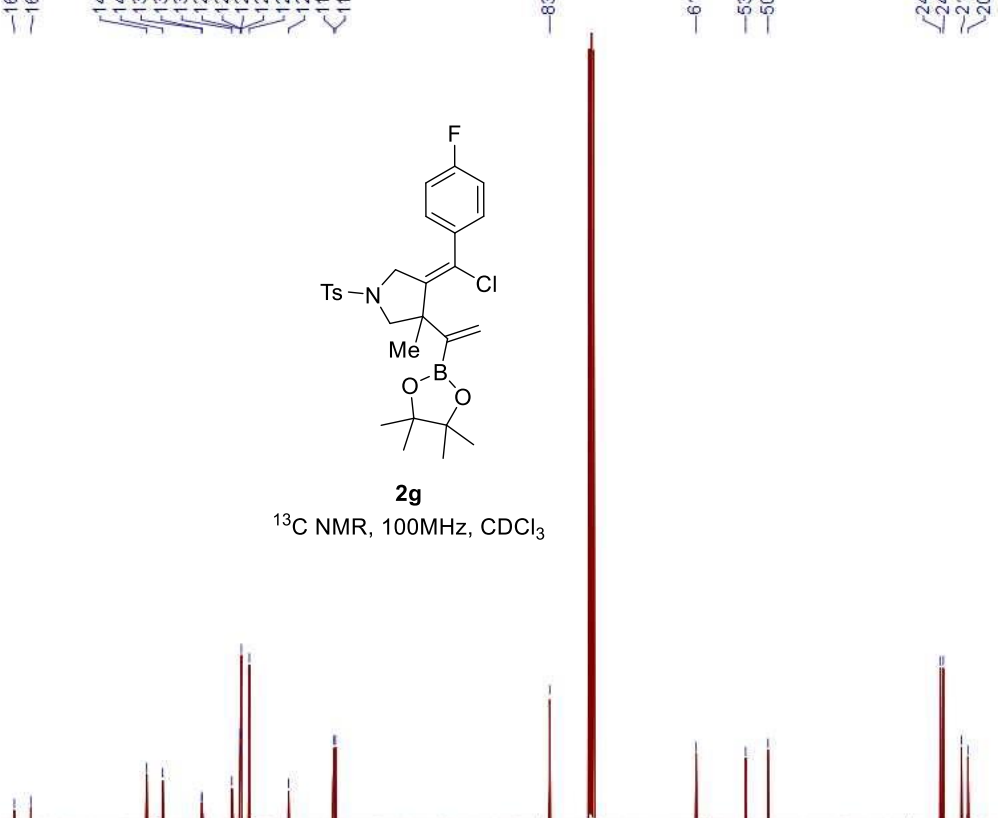


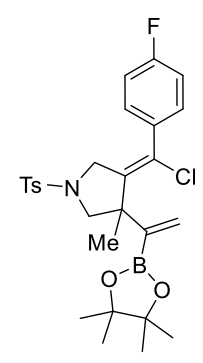

${ }^{11} \mathrm{~B}$ NMR, $128 \mathrm{MHz}, \mathrm{CDCl}_{3}$

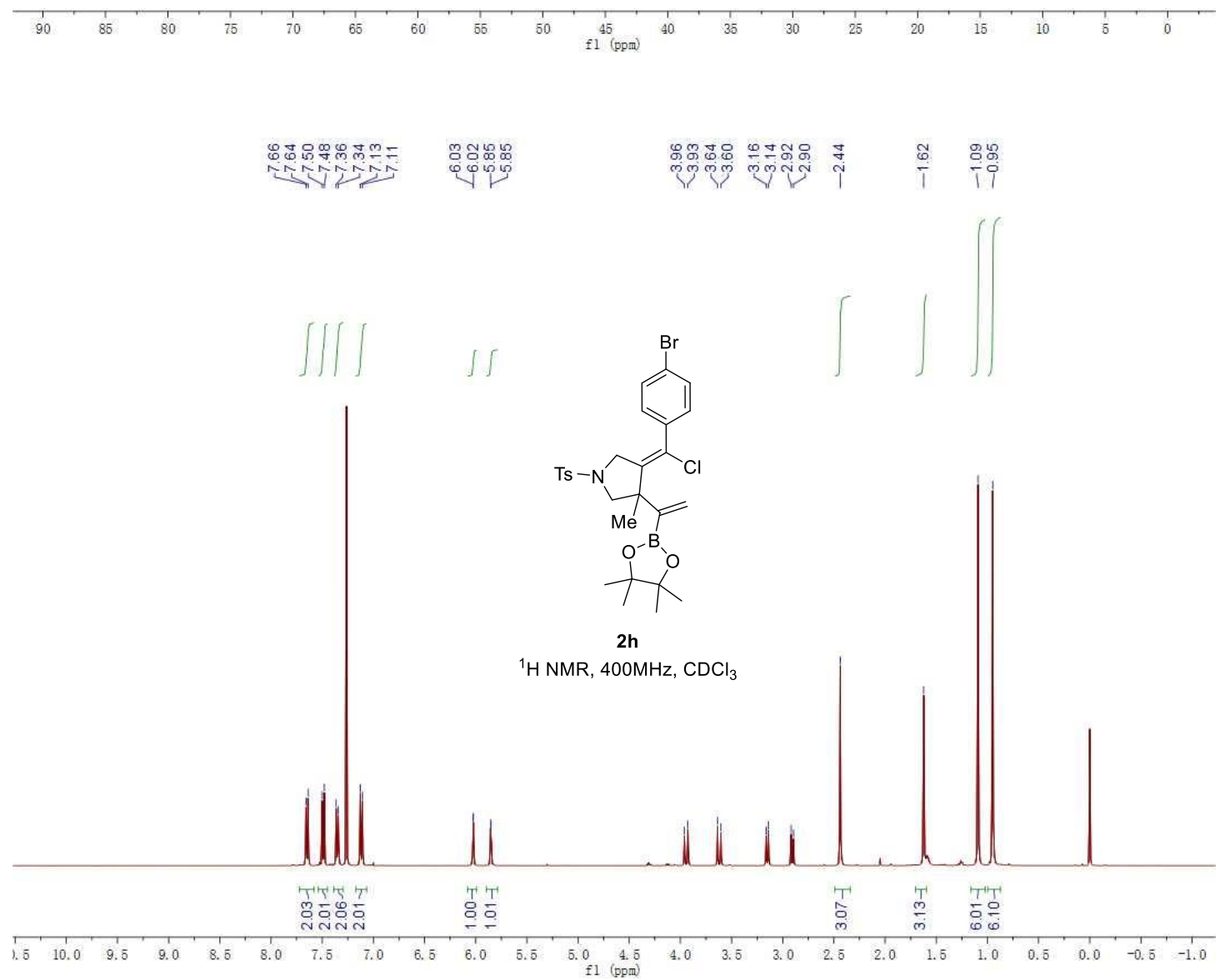




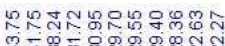

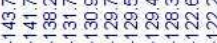

$$
\begin{aligned}
& 2 \\
& \substack{2 \\
1 \\
1} \\
& 1
\end{aligned}
$$

సิ

89

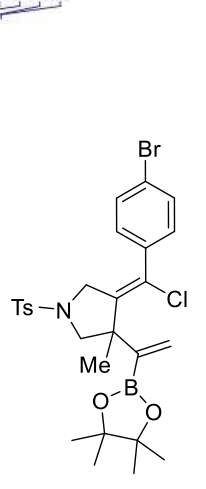

2h

${ }^{13} \mathrm{C} \mathrm{NMR}, 100 \mathrm{MHz}, \mathrm{CDCl}_{3}$
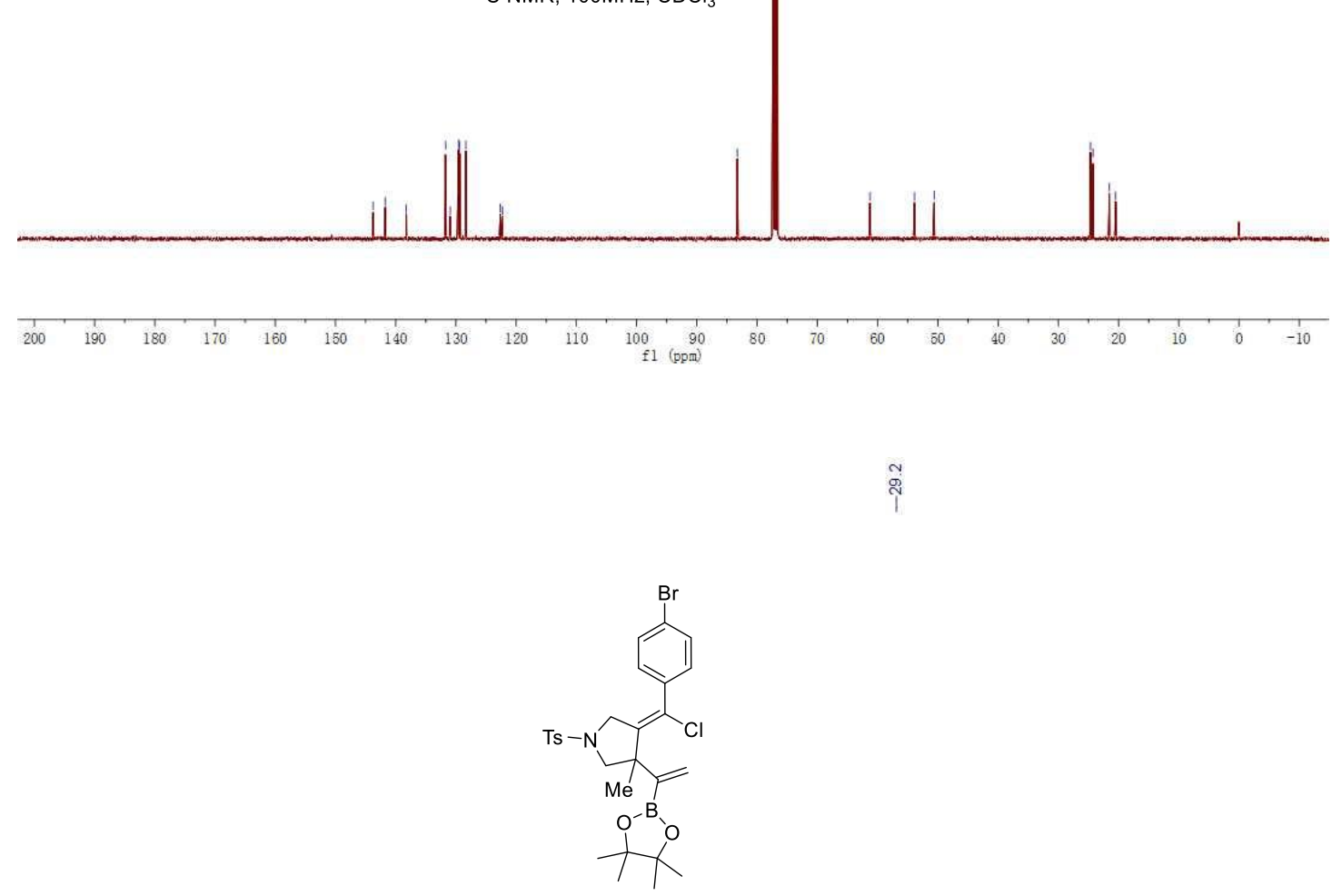

2h

${ }^{11} \mathrm{~B}$ NMR, $128 \mathrm{MHz}, \mathrm{CDCl}_{3}$

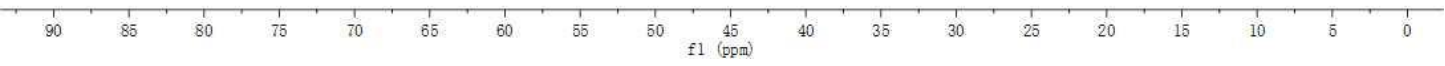




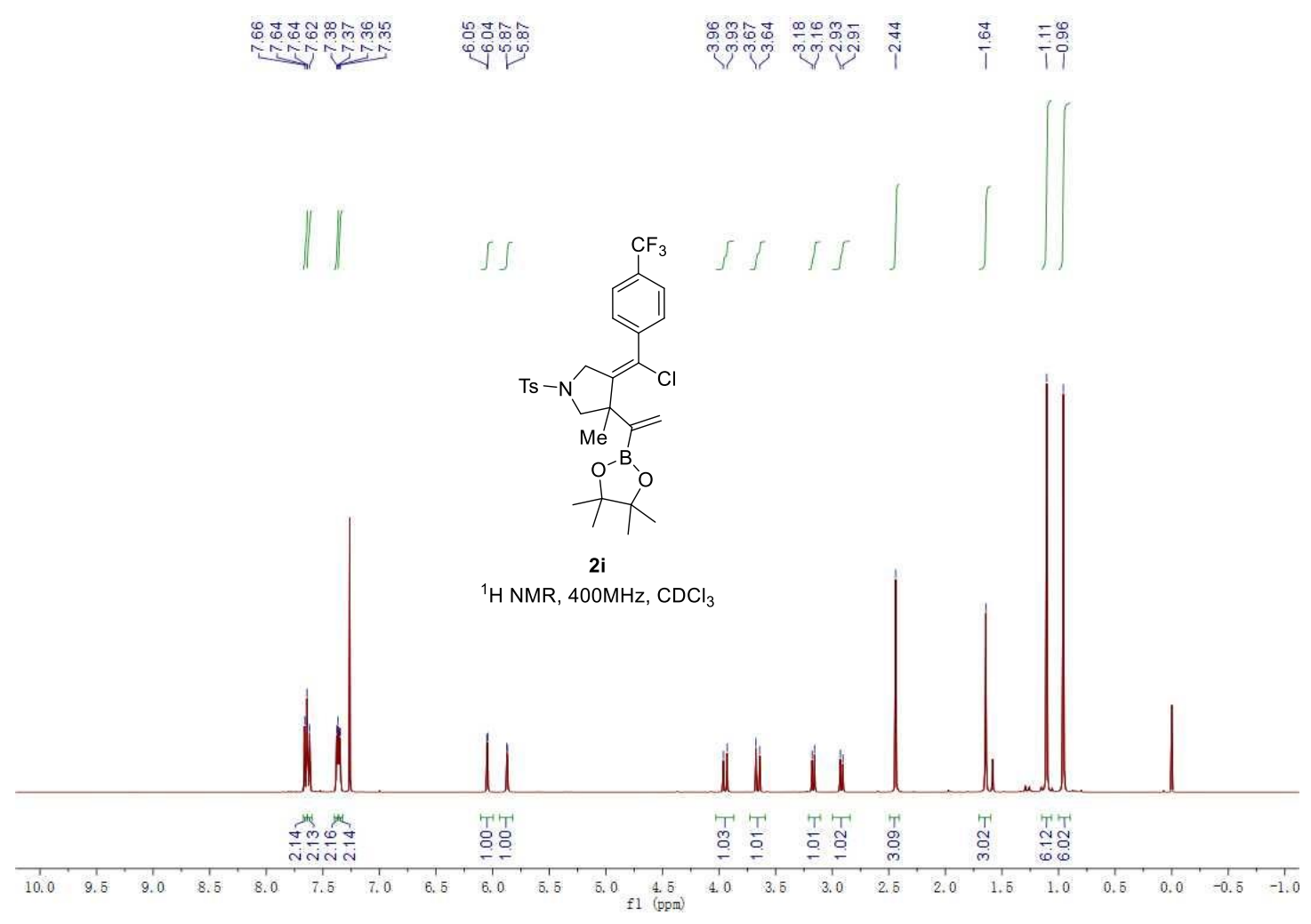

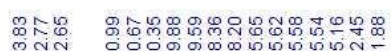

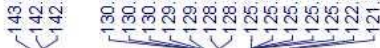

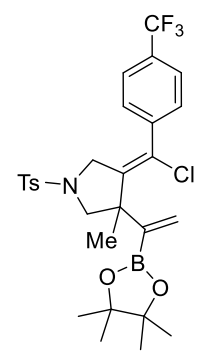

2i

${ }^{13} \mathrm{C}$ NMR, $100 \mathrm{MHz}, \mathrm{CDCl}_{3}$

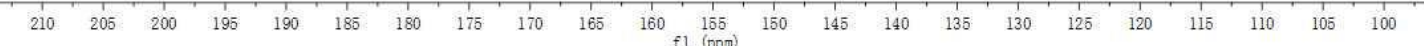




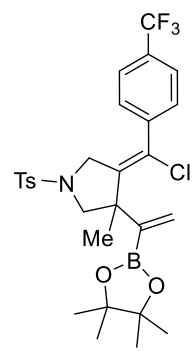

$\stackrel{\infty}{7}$

${ }^{11} \mathrm{~B} \mathrm{NMR}, 128 \mathrm{MHz}, \mathrm{CDCl}_{3}$
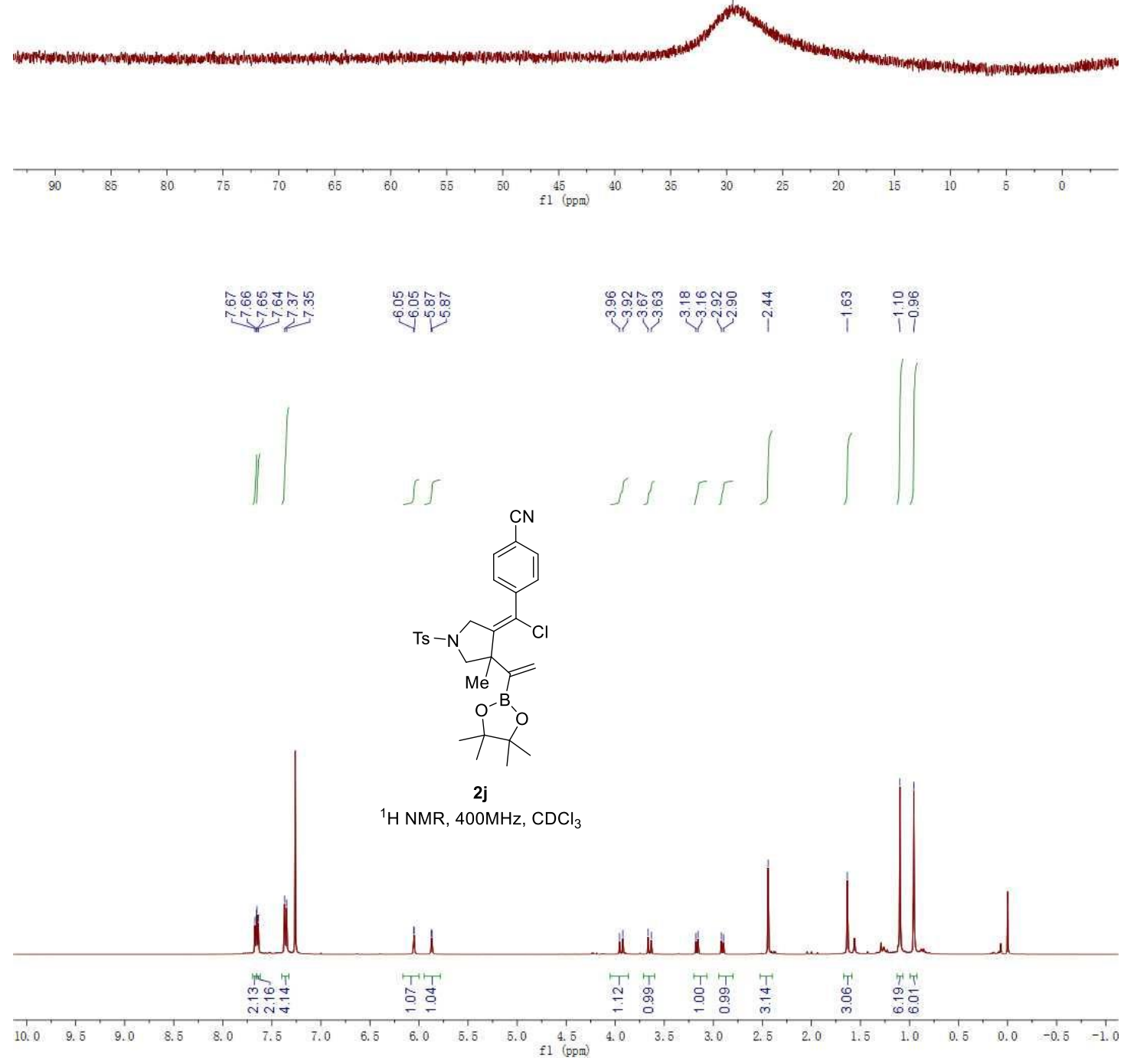

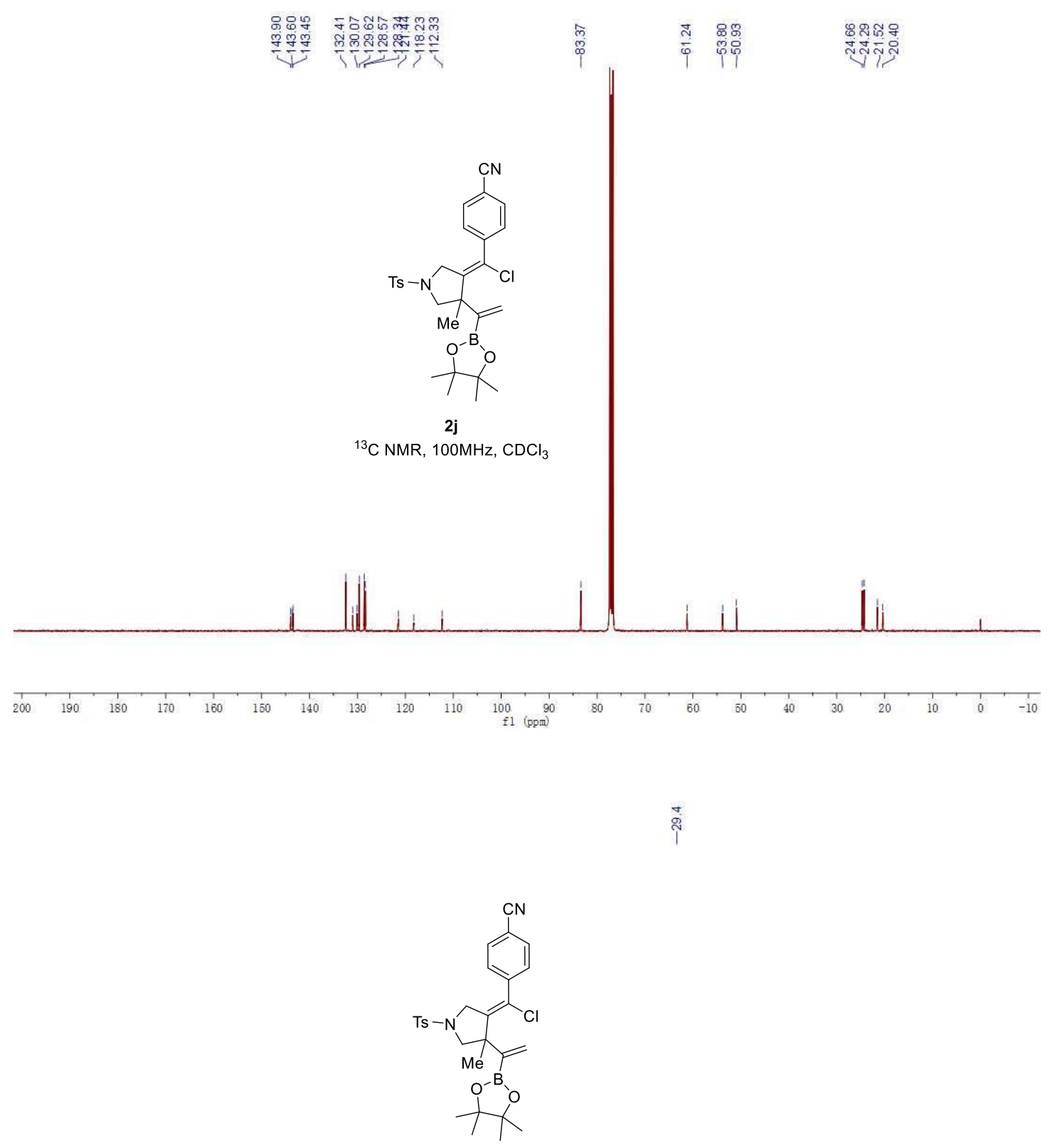

${ }^{11} \mathrm{~B}$ NMR, $128 \mathrm{MHz}, \mathrm{CDCl}_{3}$

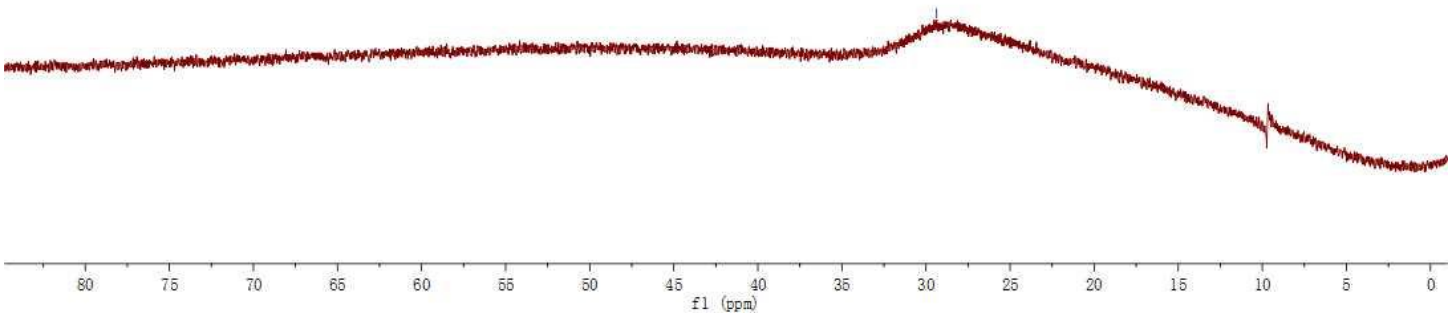




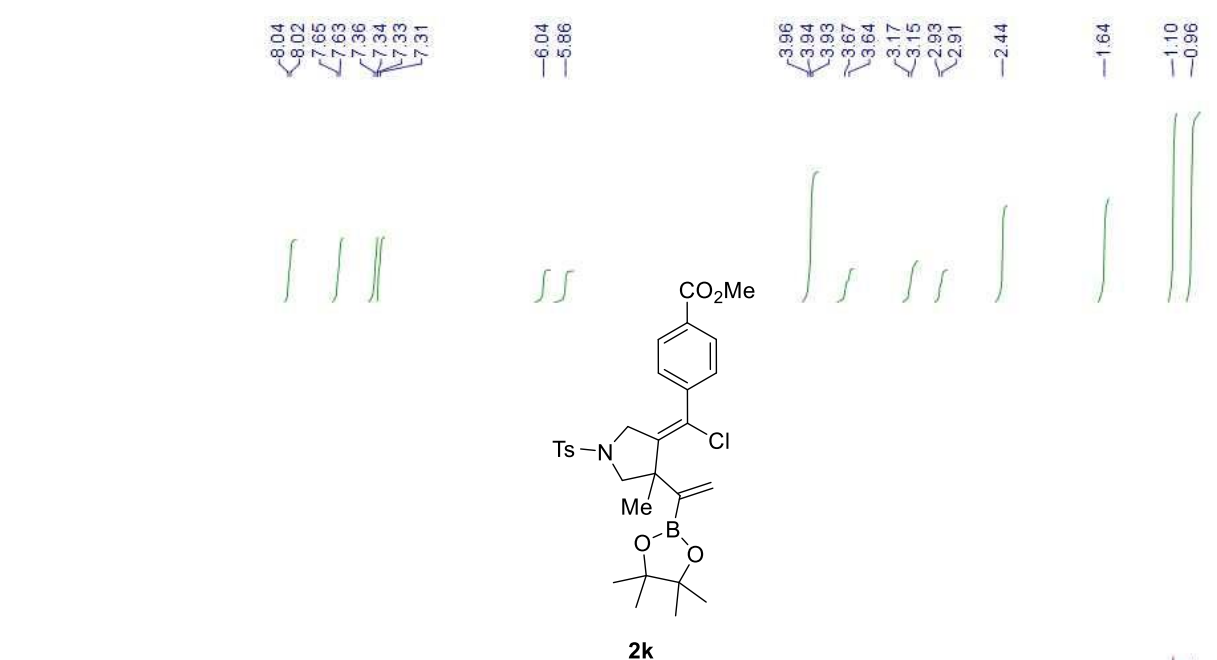

${ }^{1} \mathrm{H} \mathrm{NMR}, 400 \mathrm{MHz}, \mathrm{CDCl}_{3}$
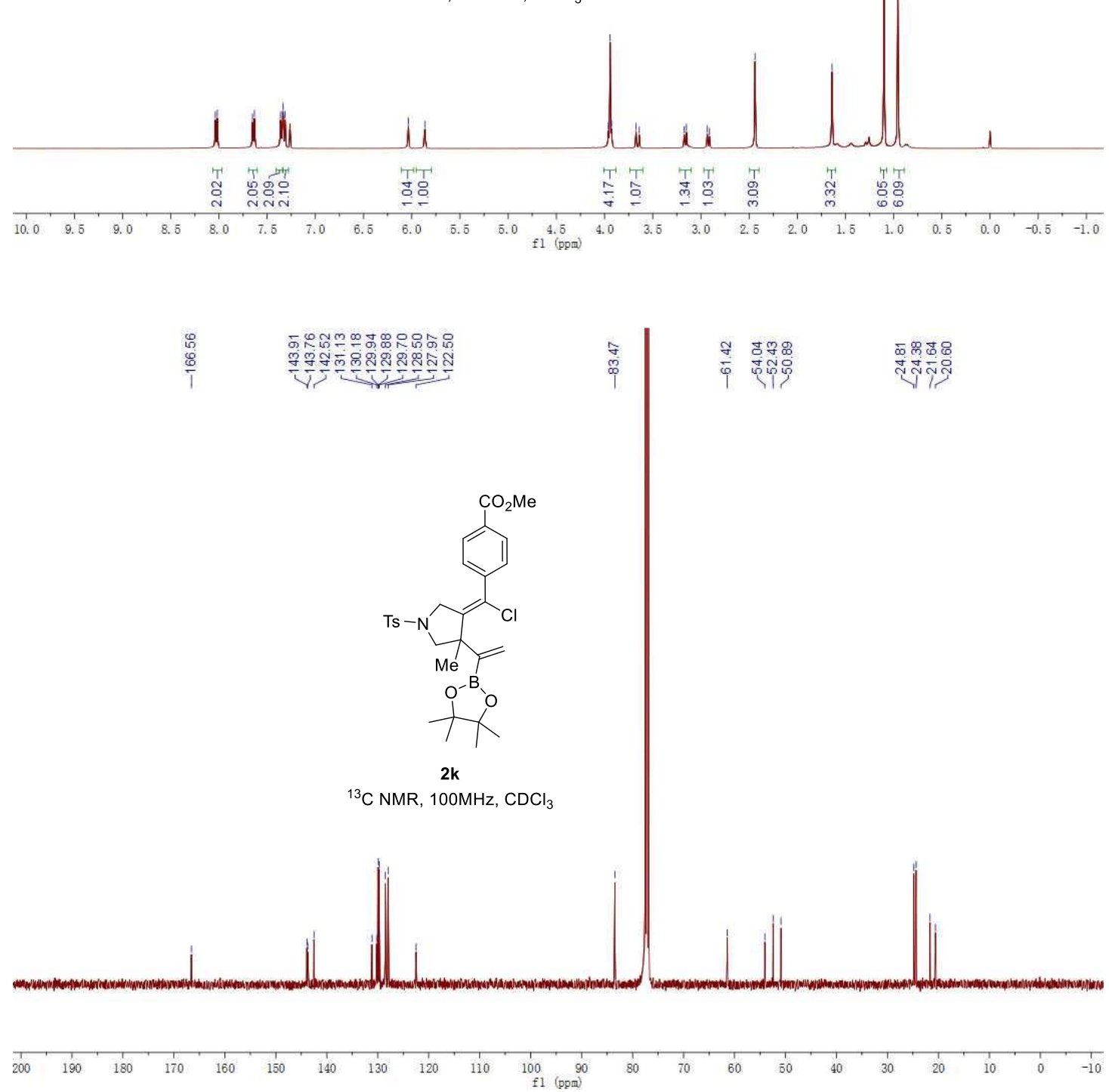


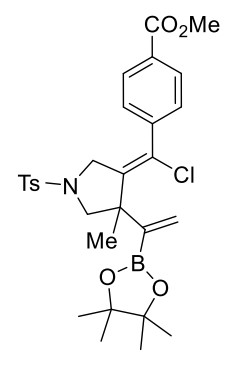

${ }^{11} \mathrm{~B} \mathrm{NMR}, 128 \mathrm{MHz}, \mathrm{CDCl}_{3}$

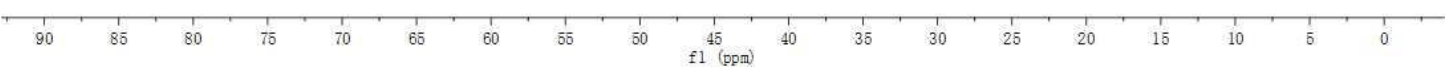

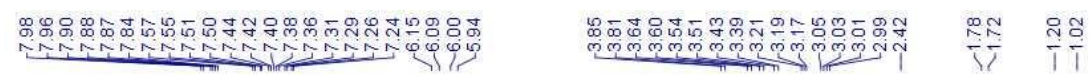
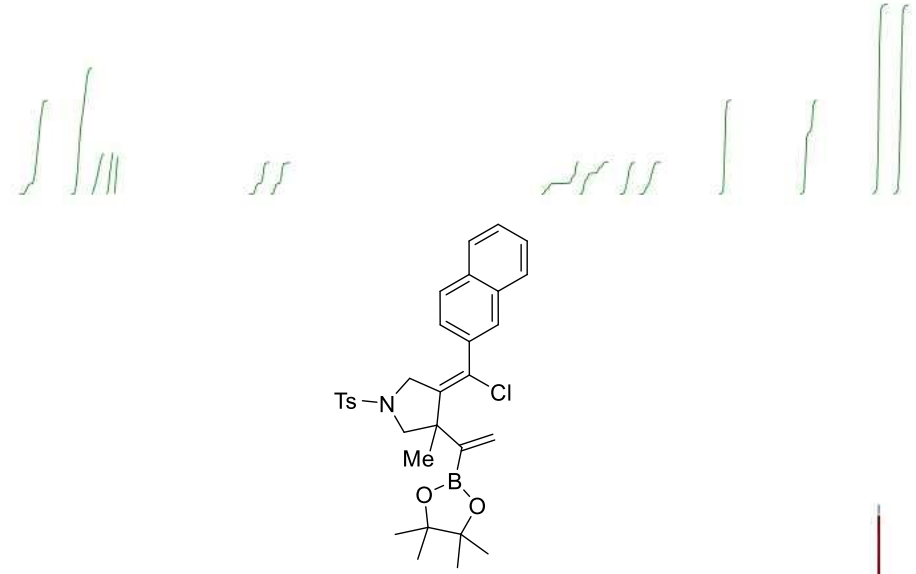

21

${ }^{1} \mathrm{H} \mathrm{NMR}, 400 \mathrm{MHz}, \mathrm{CDCl}_{3}$

Nhath

Whathend

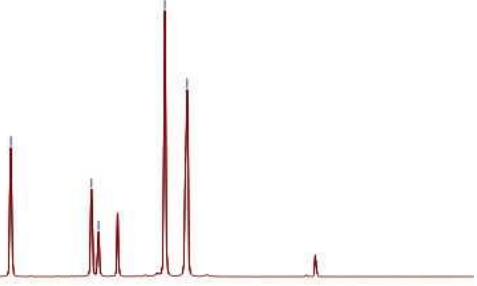

雪

Mil

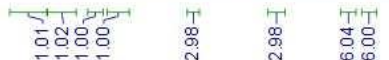

10.

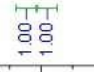

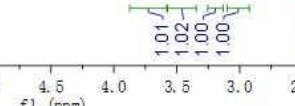

. 2015 

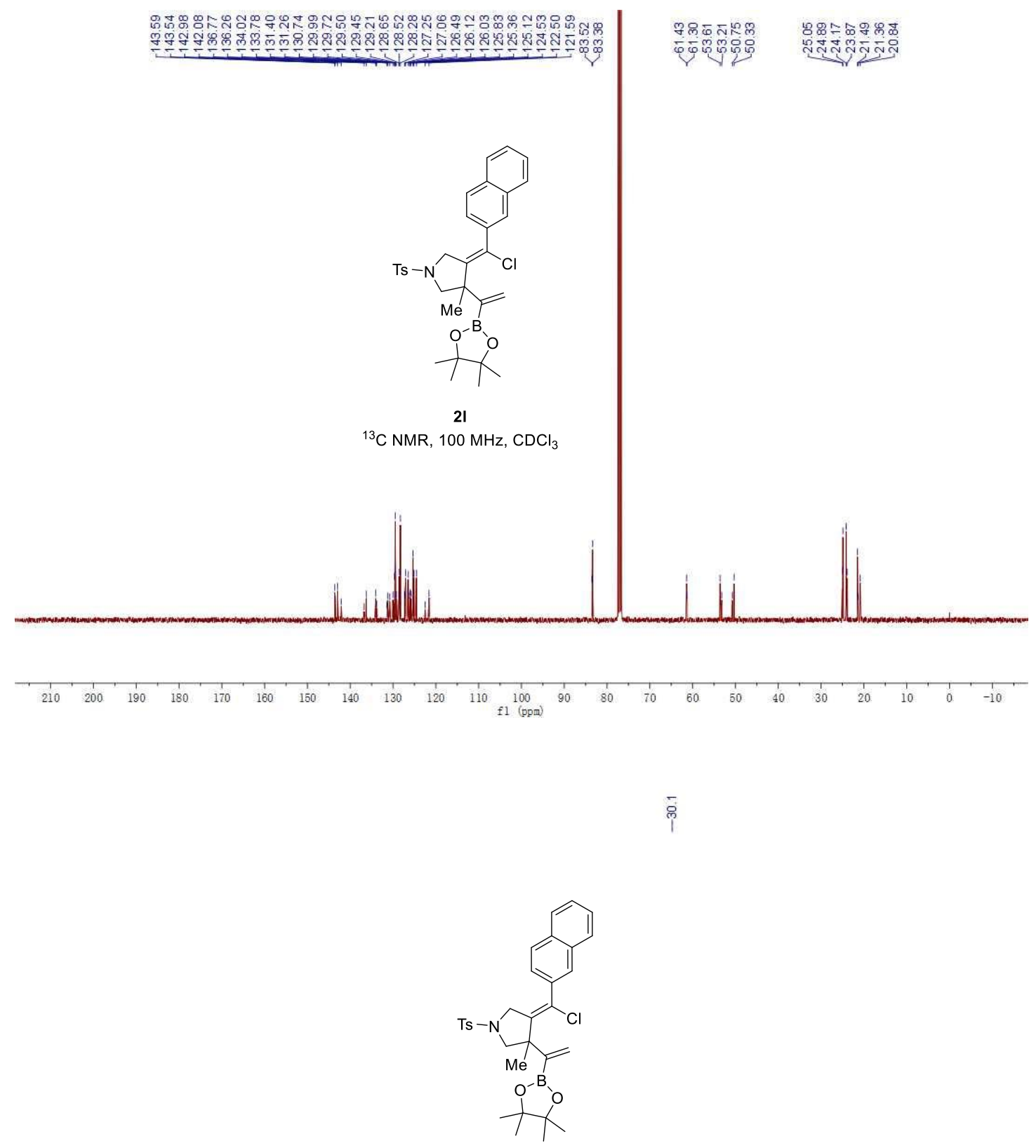

21

${ }^{11} \mathrm{~B}$ NMR, $128 \mathrm{MHz}, \mathrm{CDCl}_{3}$

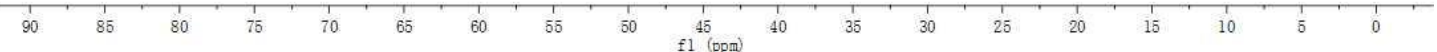




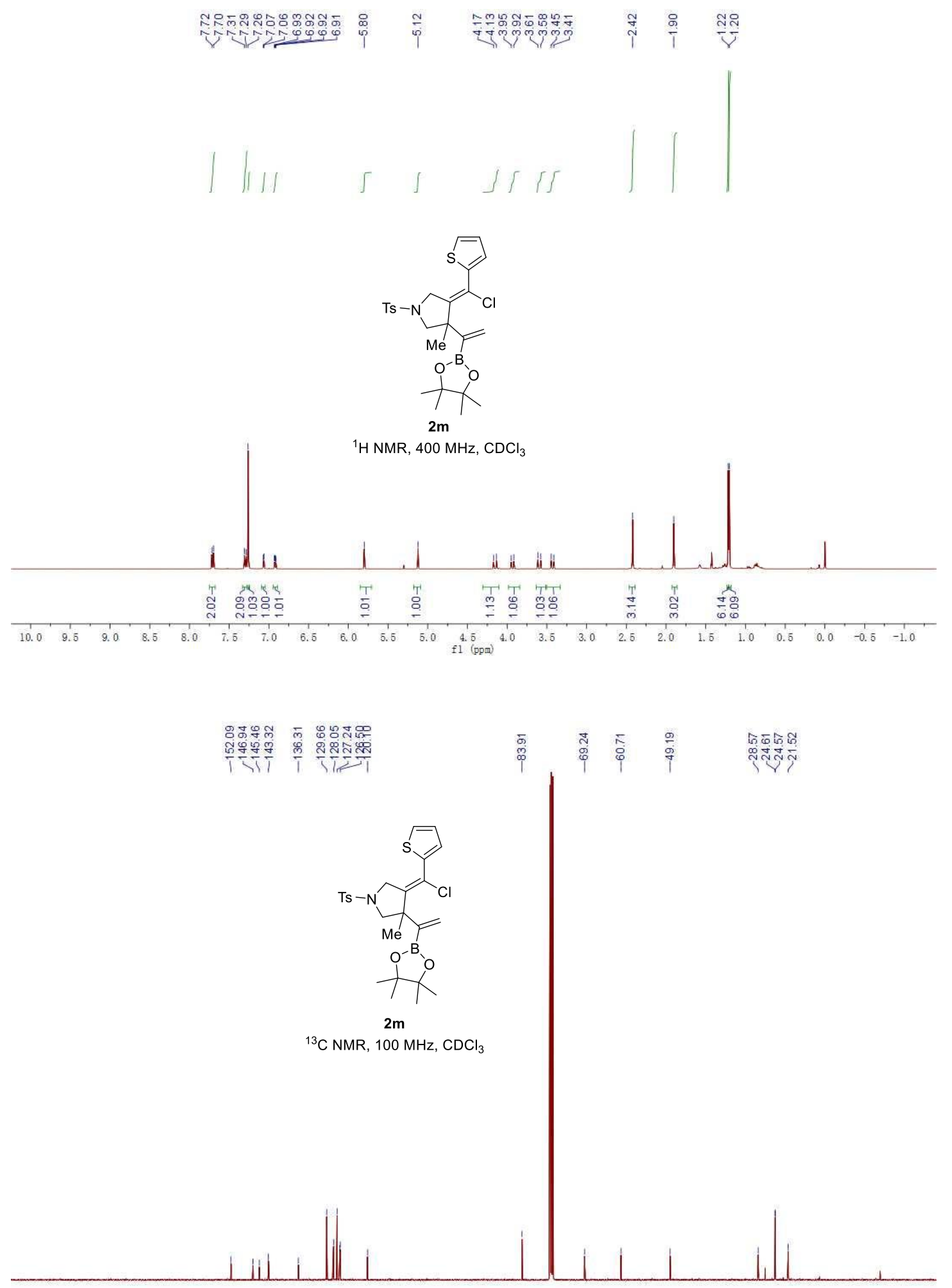

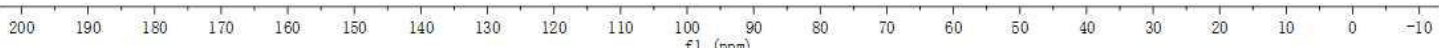




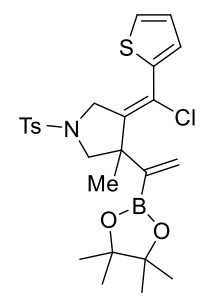

$2 \mathrm{~m}$

${ }^{11} \mathrm{~B}$ NMR, $128 \mathrm{MHz}, \mathrm{CDCl}_{3}$
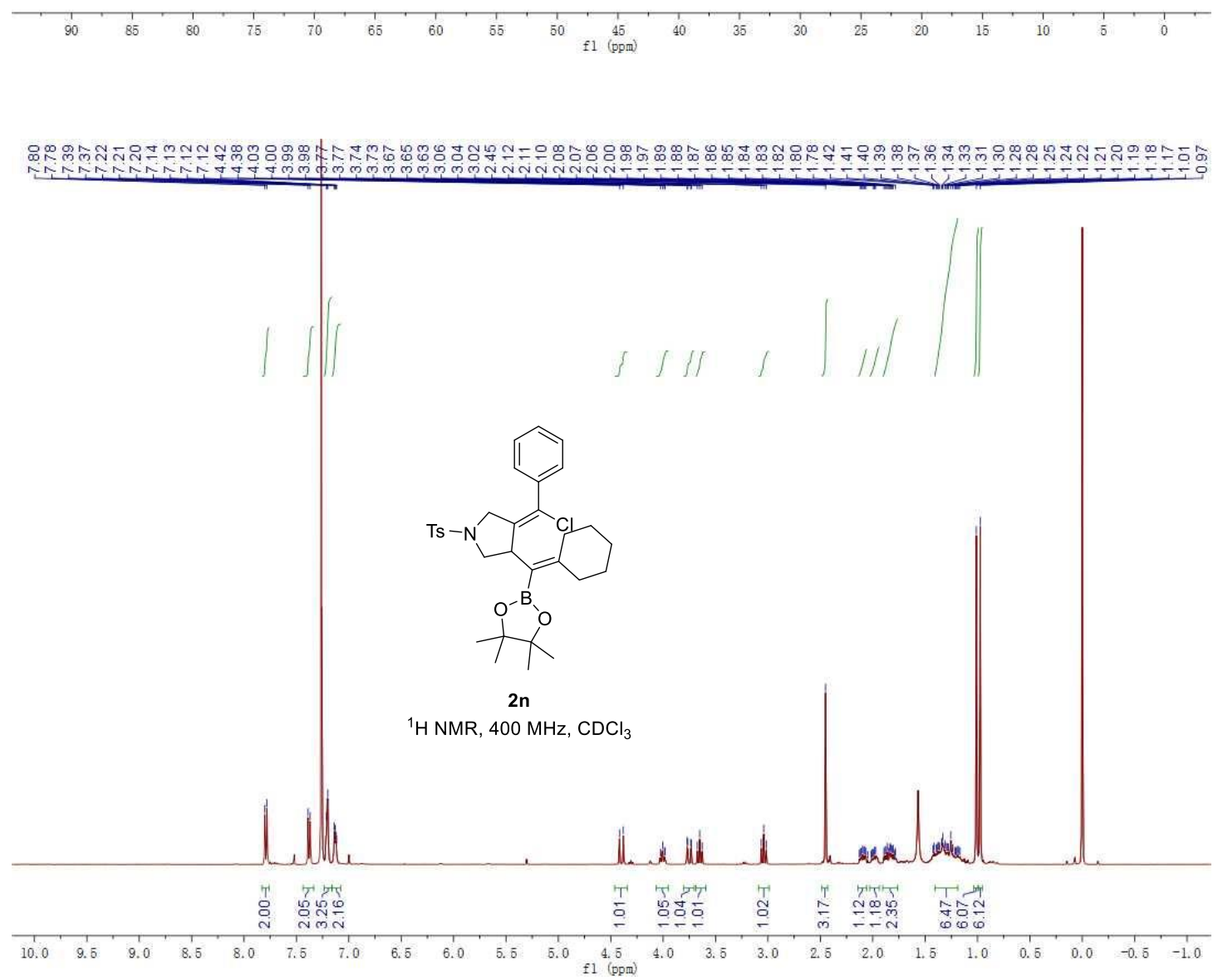

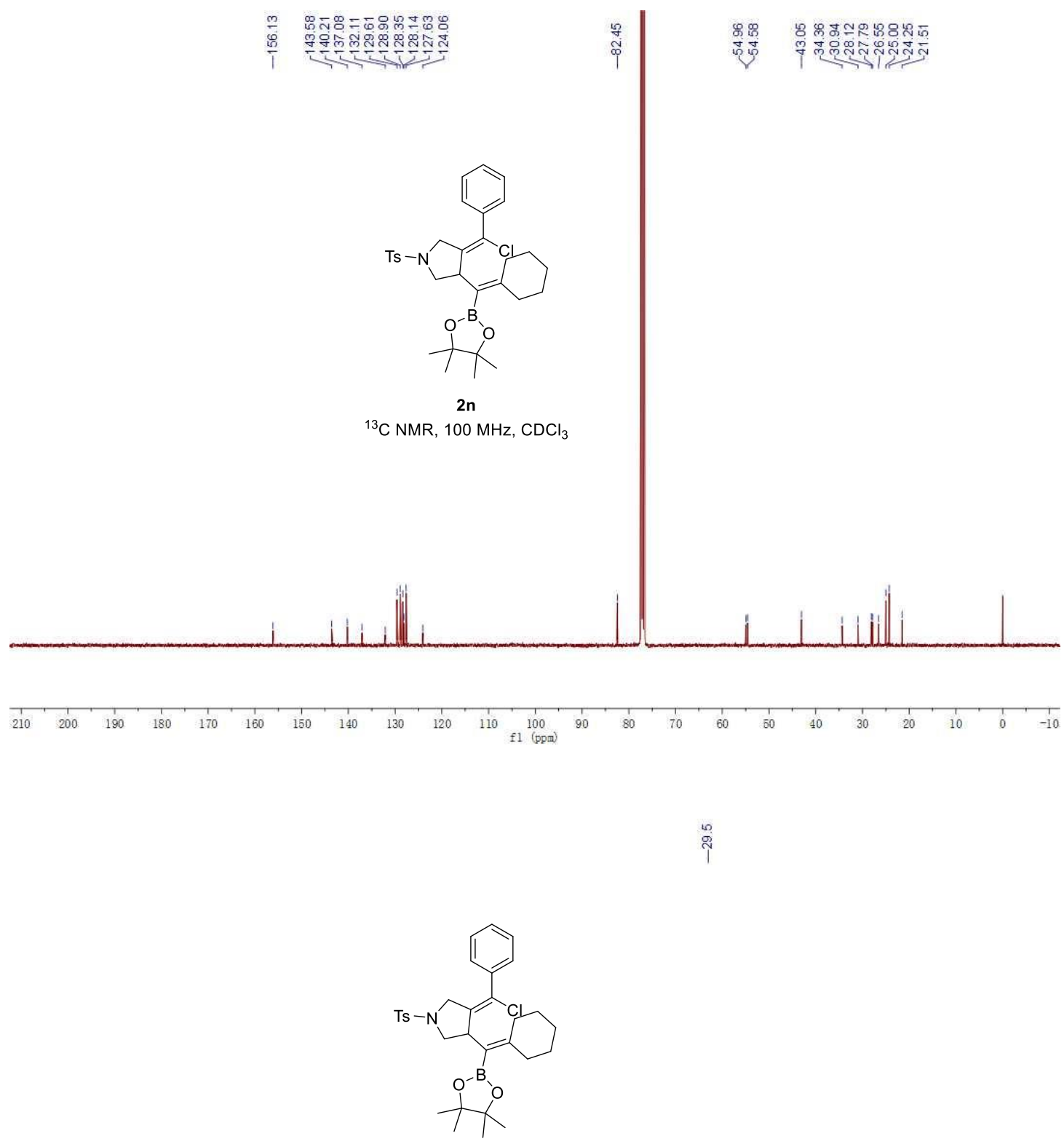

${ }^{11} \mathrm{~B} \mathrm{NMR}, 128 \mathrm{MHz}, \mathrm{CDCl}_{3}$

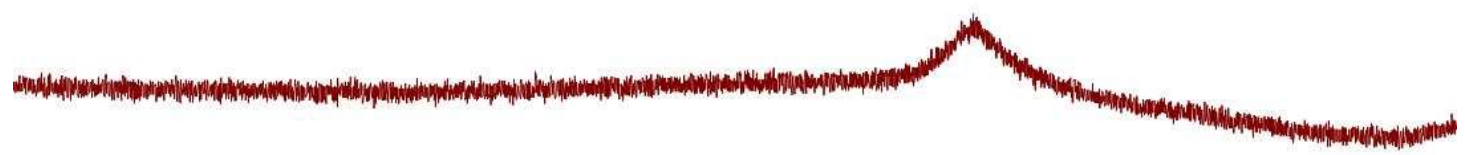

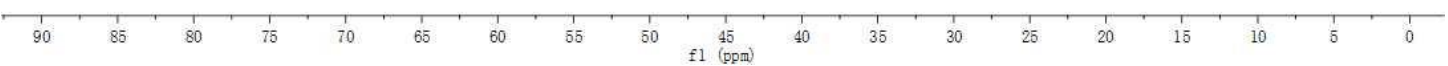




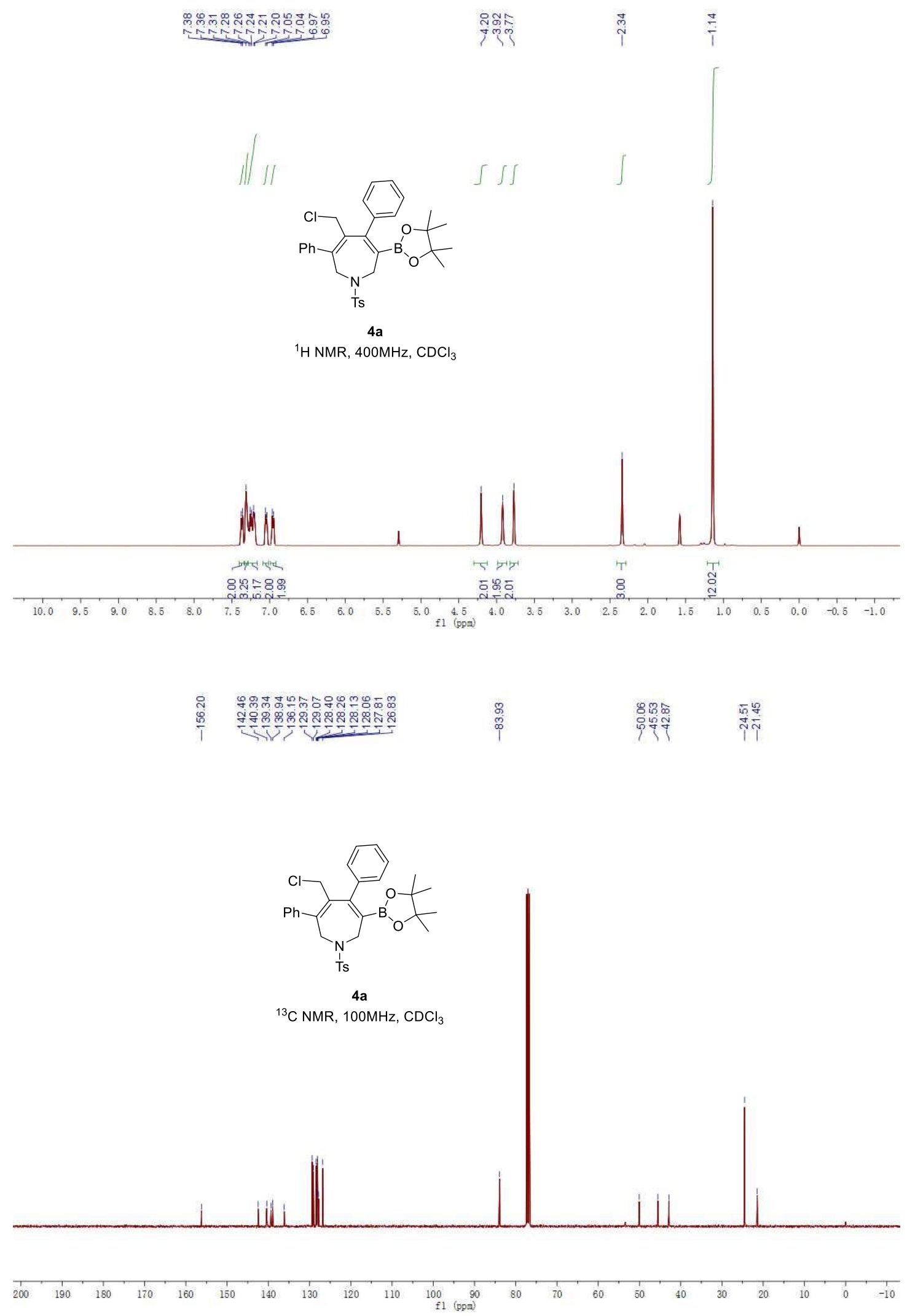




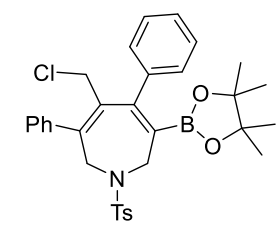

$4 a$

${ }^{11} \mathrm{~B} N M R, 128 \mathrm{MHz}, \mathrm{CDCl}_{3}$
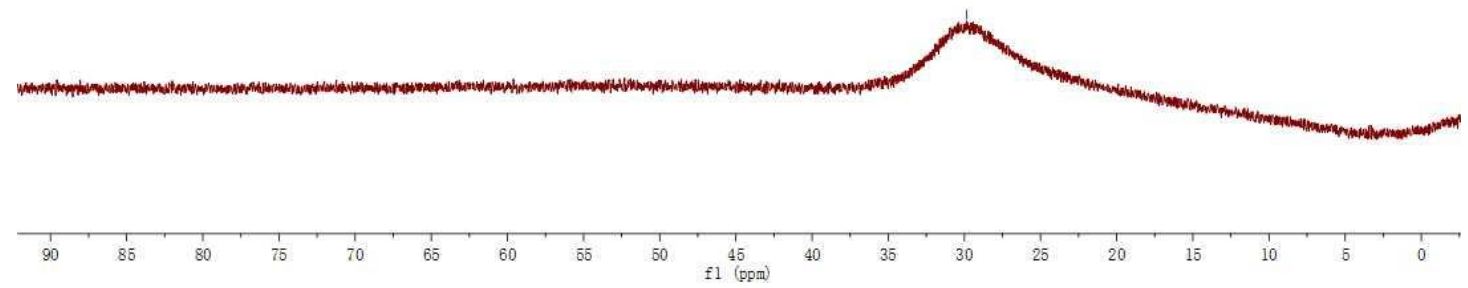

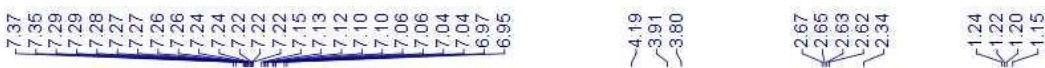
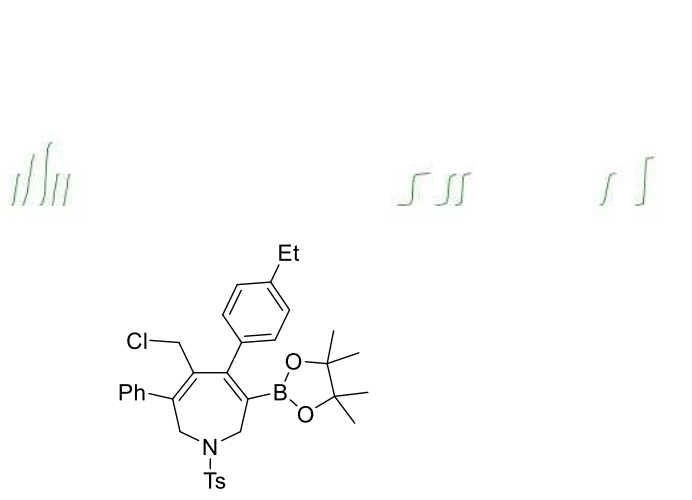

4b

${ }^{1} \mathrm{H} \mathrm{NMR}, 400 \mathrm{MHz}, \mathrm{CDCl}_{3}$

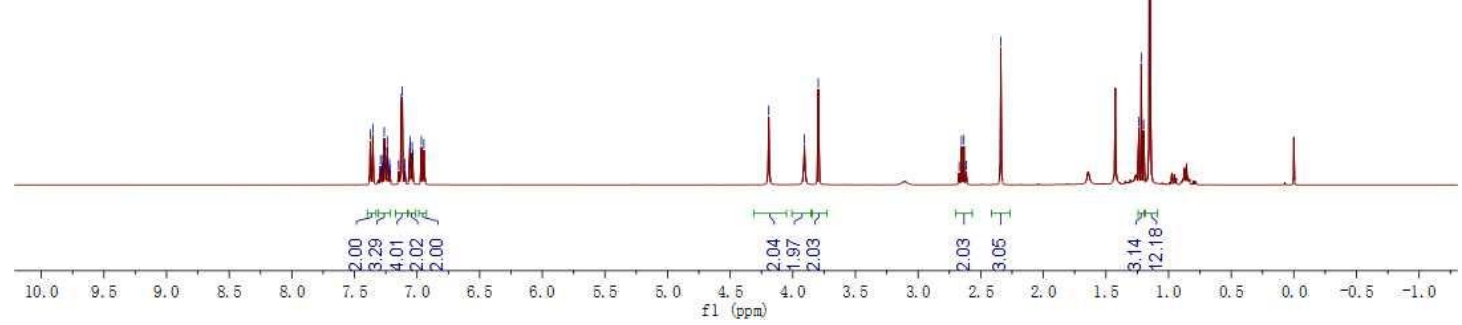




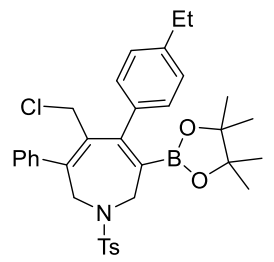

4b

${ }^{13} \mathrm{C} \mathrm{NMR}, 100 \mathrm{MHz}, \mathrm{CDCl}_{3}$
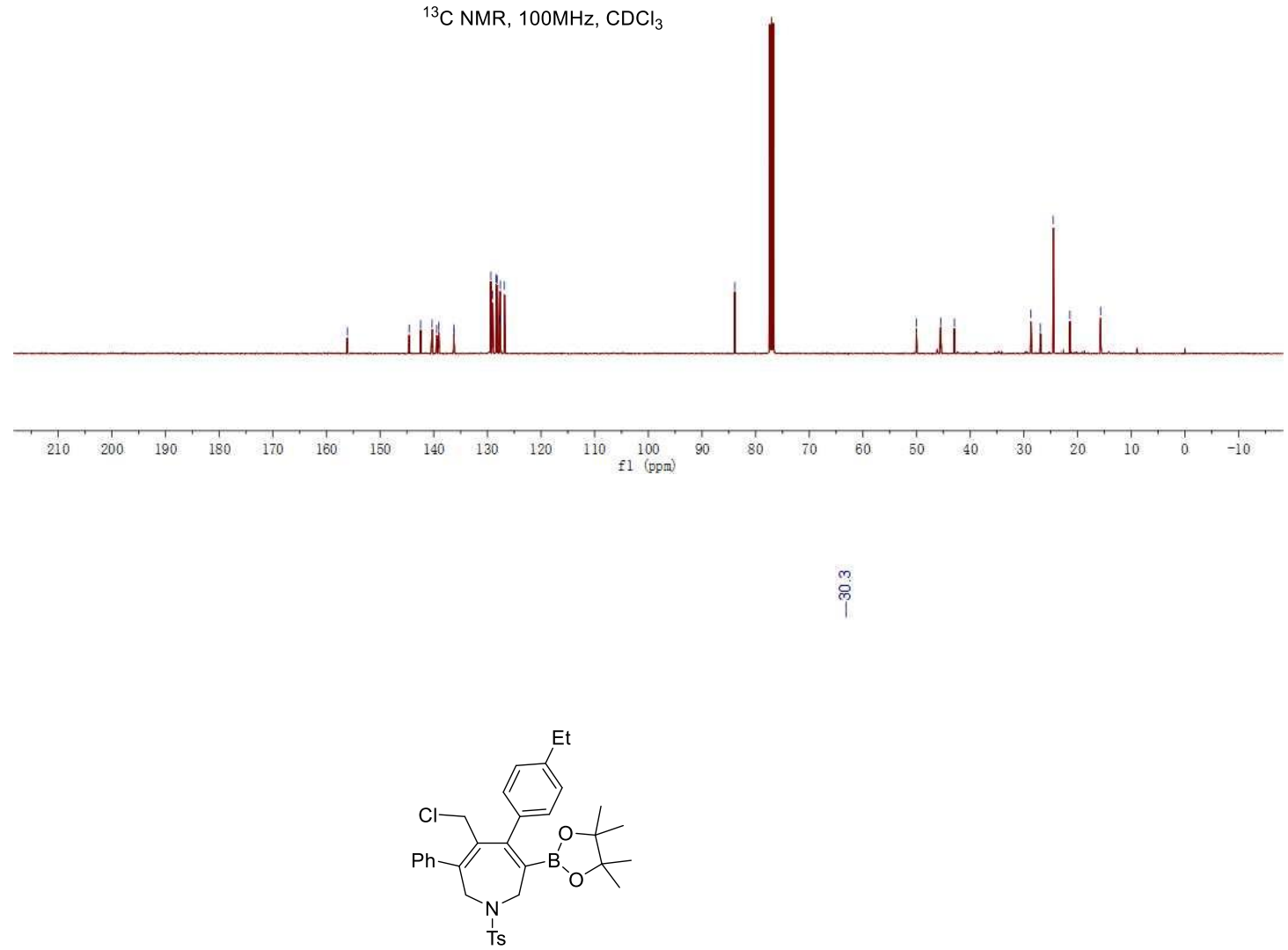

4b

${ }^{11} \mathrm{~B} \mathrm{NMR}, 128 \mathrm{MHz}, \mathrm{CDCl}_{3}$

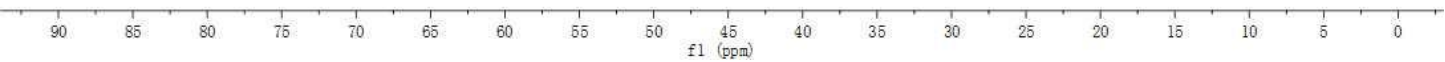




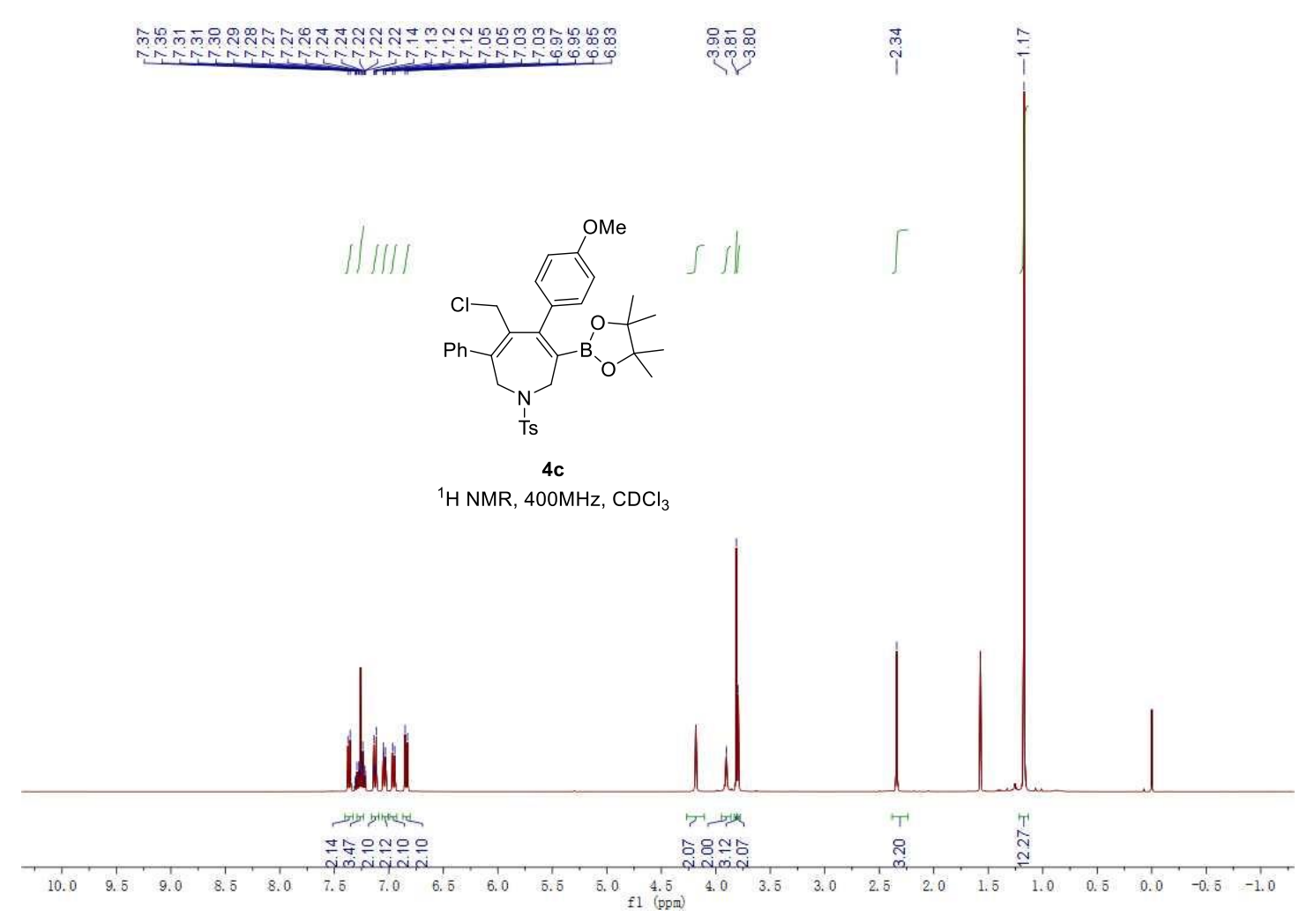

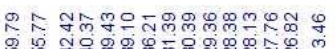

।

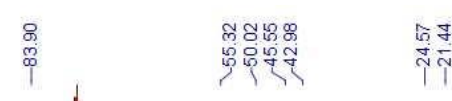

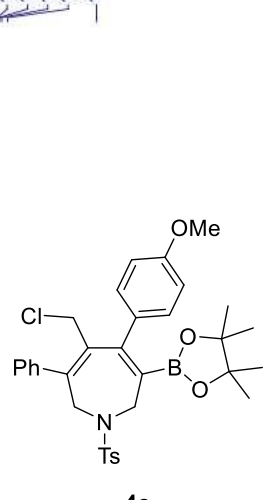

$4 c$

${ }^{13} \mathrm{C} \mathrm{NMR}, 100 \mathrm{MHz}, \mathrm{CDCl}_{3}$

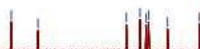
i

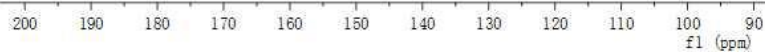




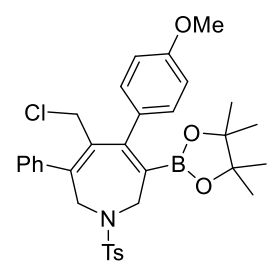

$4 \mathrm{c}$

${ }^{11} \mathrm{~B}$ NMR, $128 \mathrm{MHz}, \mathrm{CDCl}_{3}$
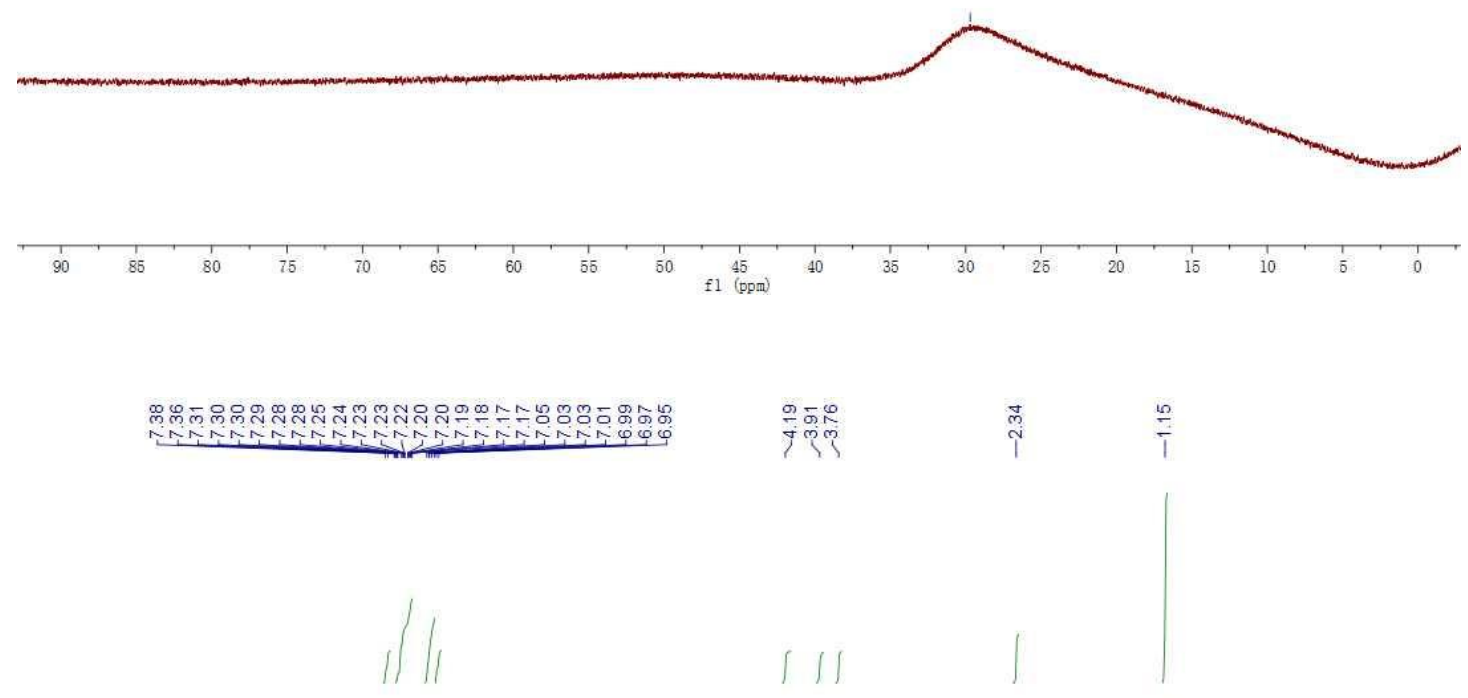

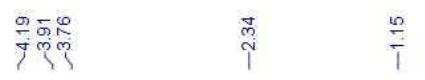

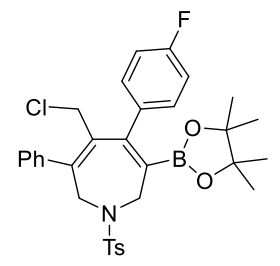

4d

${ }^{1} \mathrm{H} \mathrm{NMR}, 400 \mathrm{MHz}, \mathrm{CDCl}_{3}$

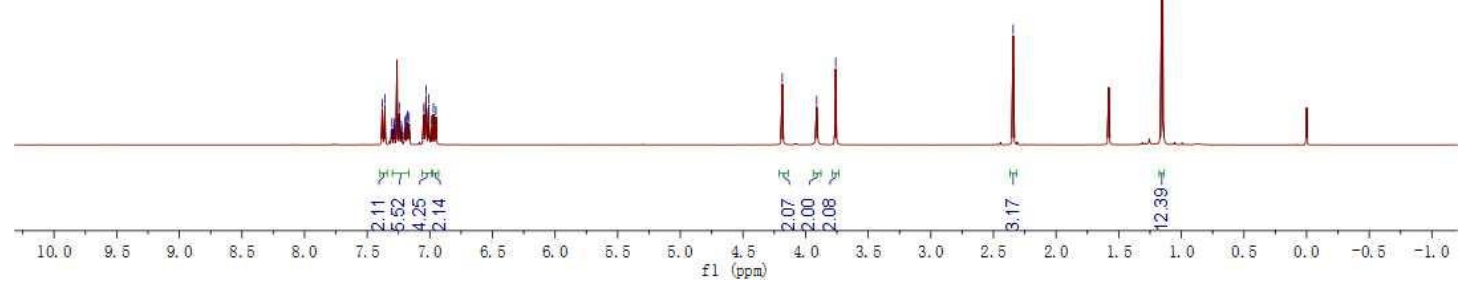




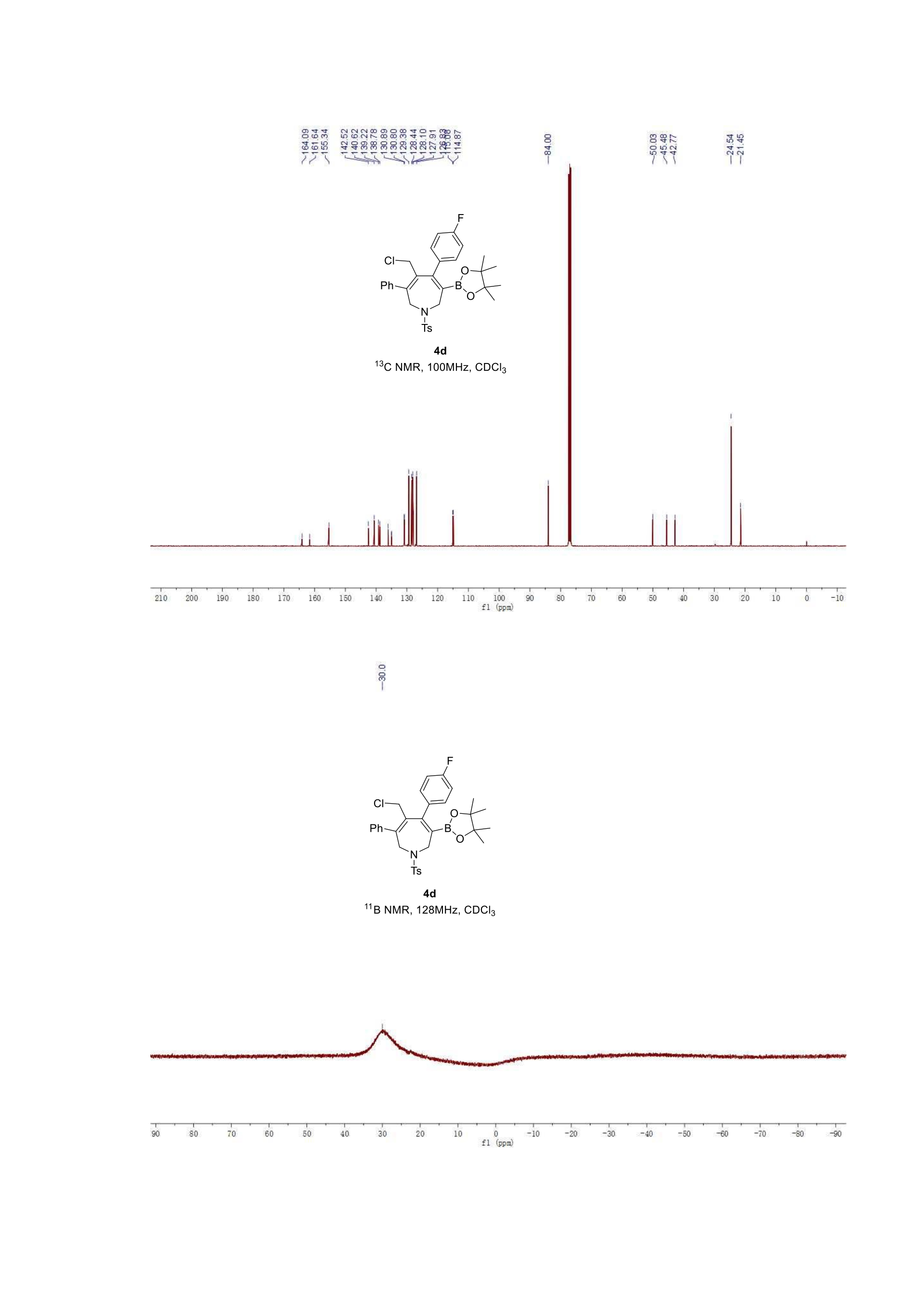




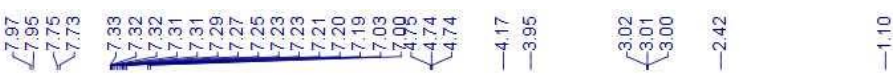
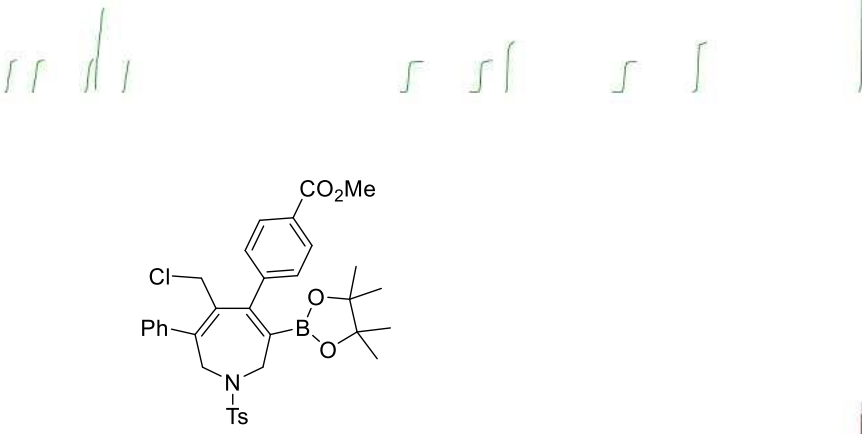

$4 \mathrm{e}$

${ }^{1} \mathrm{H} \mathrm{NMR}, 400 \mathrm{MHz}, \mathrm{CDCl}_{3}$

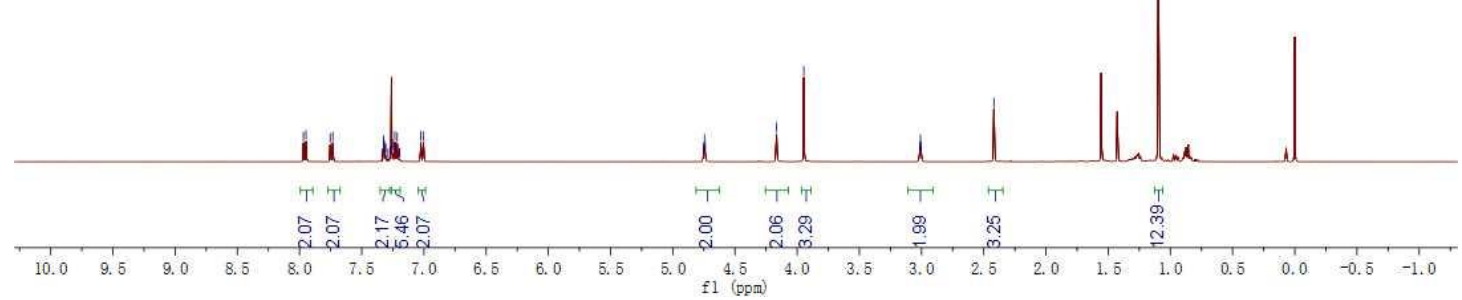

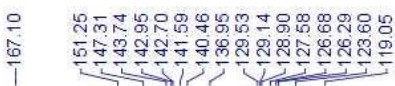
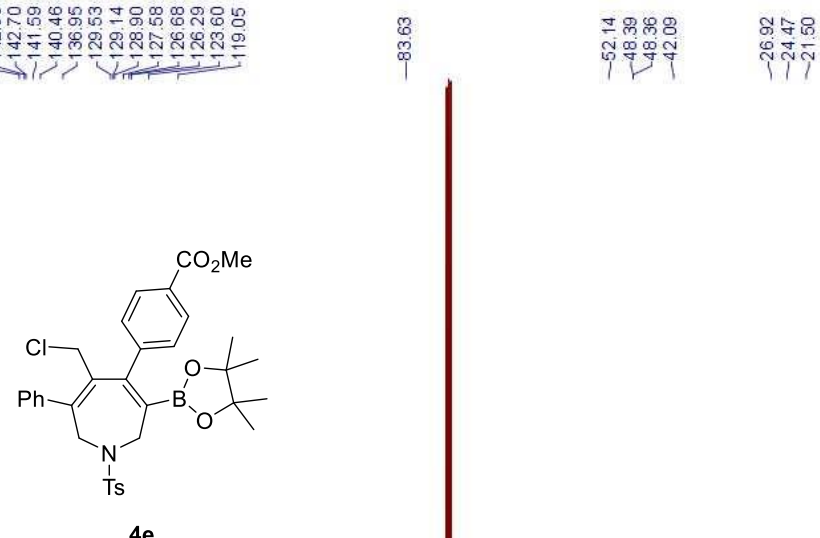

${ }^{13} \mathrm{C} \mathrm{NMR}, 100 \mathrm{MHz}, \mathrm{CDCl}_{3}$

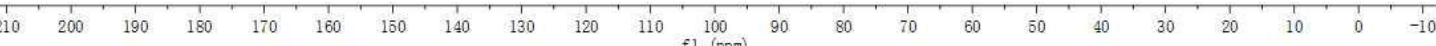




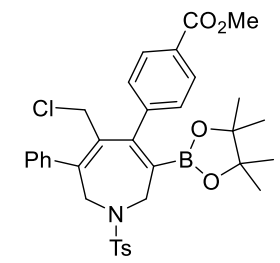

4 e

${ }^{11} \mathrm{~B}$ NMR, $128 \mathrm{MHz}, \mathrm{CDCl}_{3}$

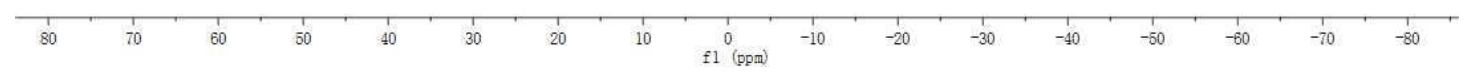

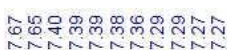

nimininin

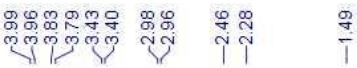
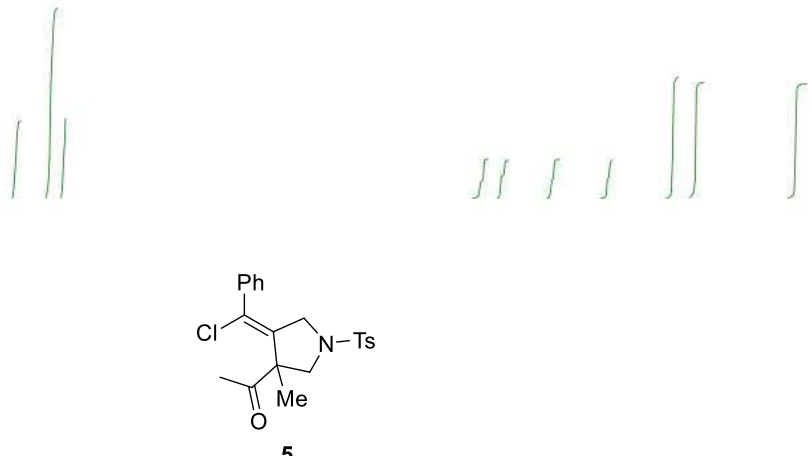

${ }^{1} \mathrm{H} \mathrm{NMR}, 400 \mathrm{MHz}, \mathrm{CDCl}_{3}$

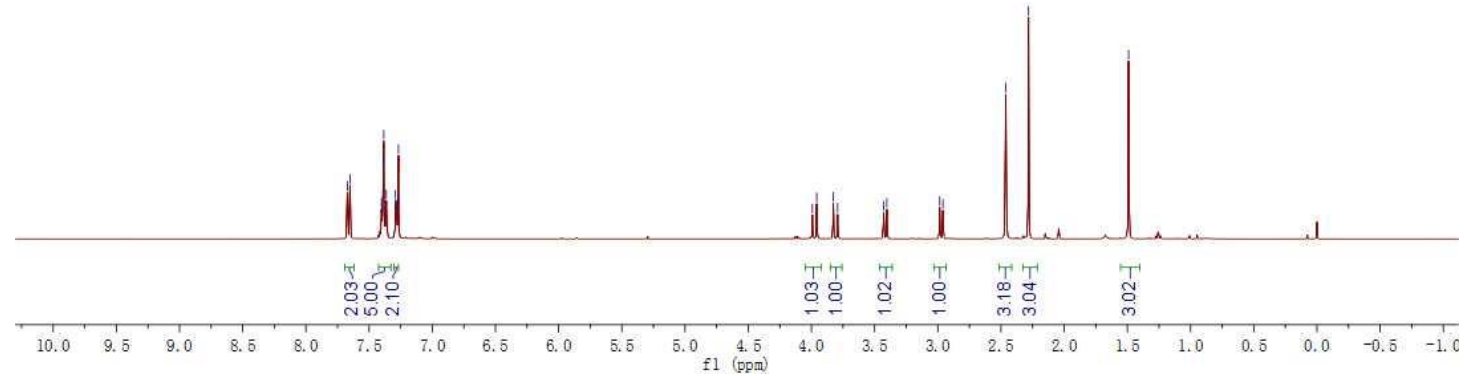




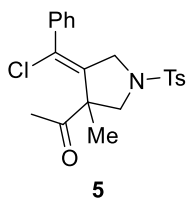

${ }^{13} \mathrm{C} \mathrm{NMR}, 100 \mathrm{MHz}, \mathrm{CDCl}_{3}$
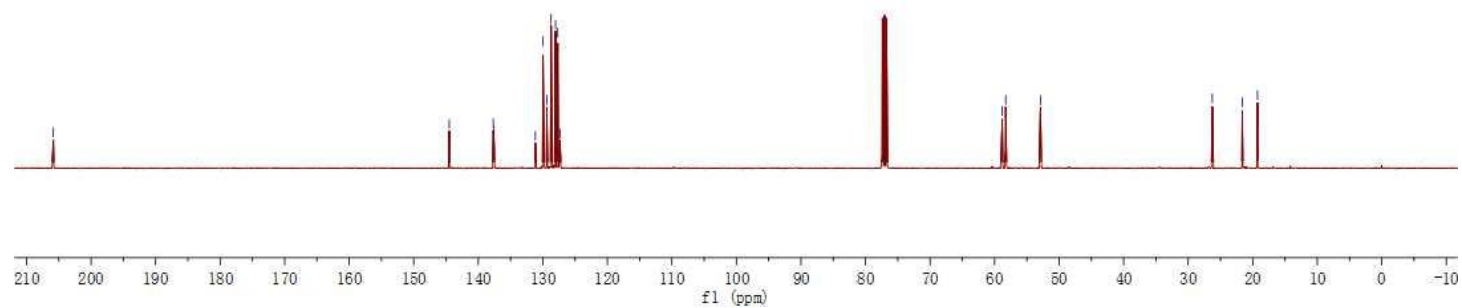

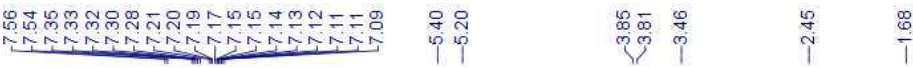
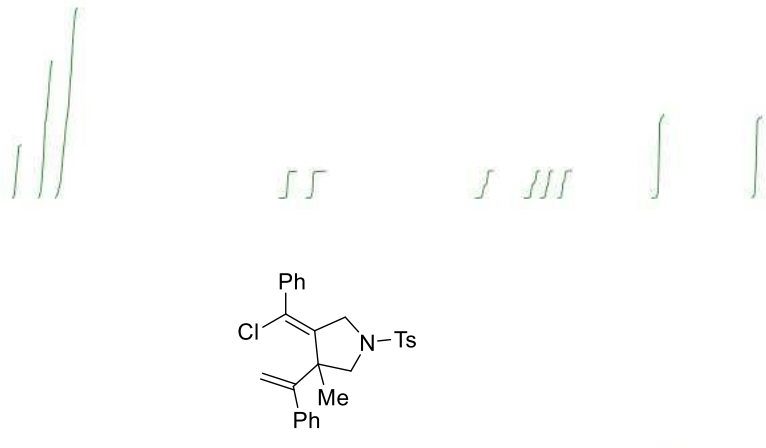

${ }^{1} \mathrm{H}$ NMR, $400 \mathrm{MHz}, \mathrm{CDCl}_{3}$

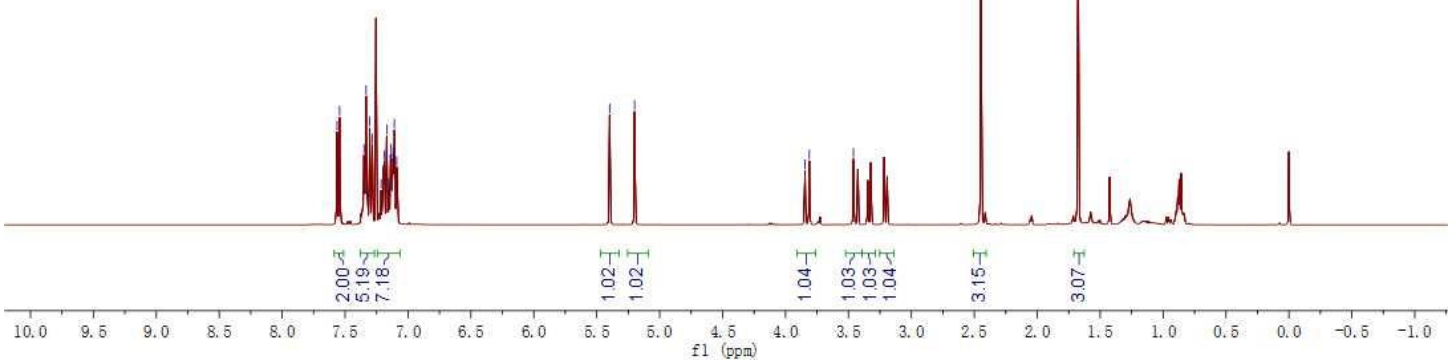




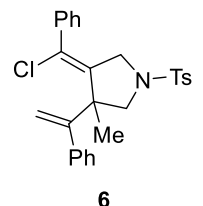

${ }^{13} \mathrm{C}$ NMR, $100 \mathrm{MHz}, \mathrm{CDCl}_{3}$
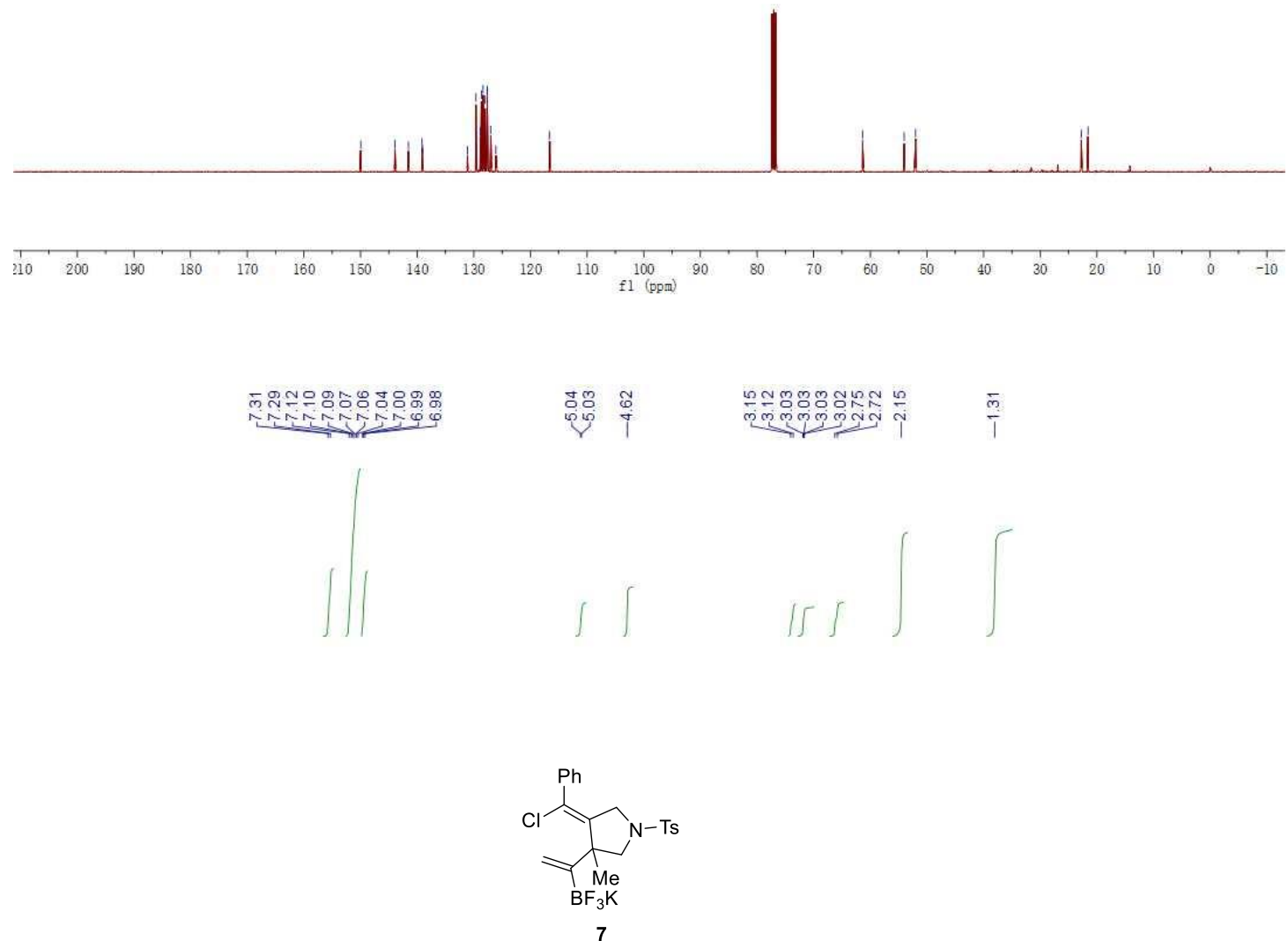

${ }^{1} \mathrm{H}$ NMR, $400 \mathrm{MHz}, \mathrm{CD}_{3} \mathrm{OD}$

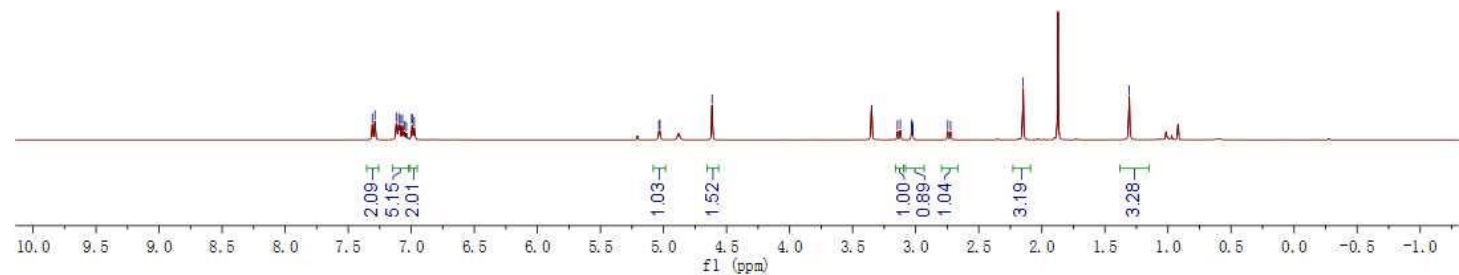




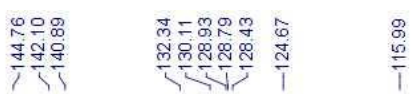

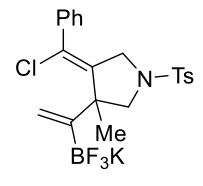

${ }^{13} \mathrm{C} \mathrm{NMR}, 100 \mathrm{MHz}, \mathrm{CD}_{3} \mathrm{OD}$
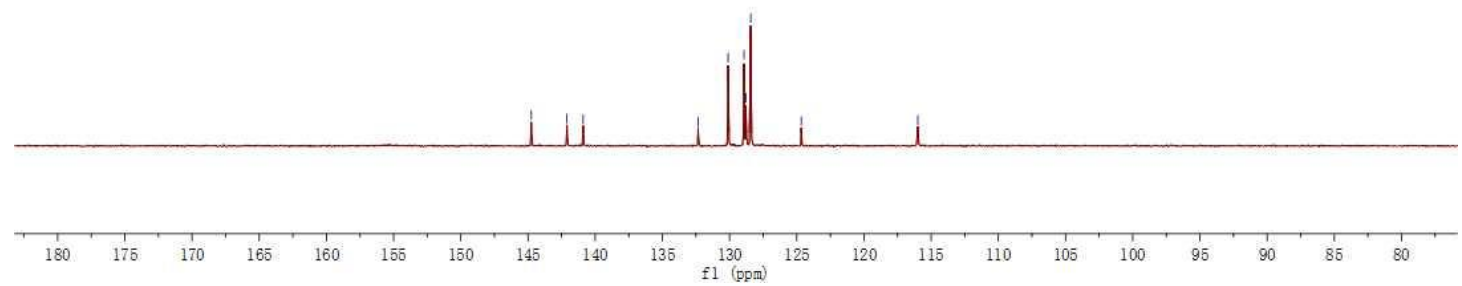

i

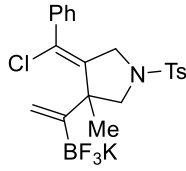

${ }^{11} \mathrm{~B} N M R, 128 \mathrm{MHz}, \mathrm{CD}_{3} \mathrm{OD}$

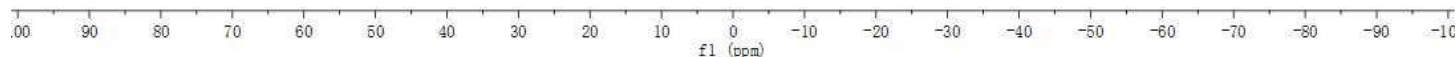


$\| \int||$
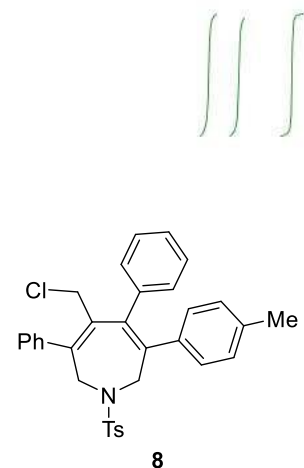

${ }^{1} \mathrm{H} \mathrm{NMR}, 400 \mathrm{MHz}, \mathrm{CDCl}_{3}$

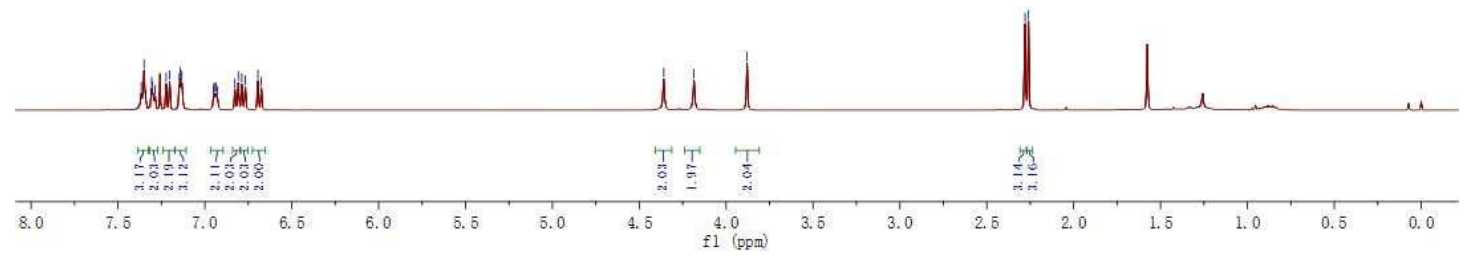

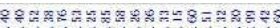

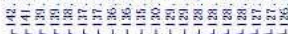

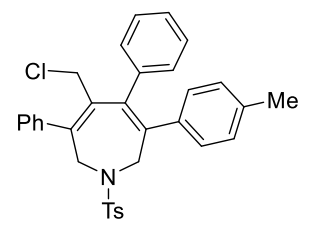

${ }^{13} \mathrm{C}$ NMR, $100 \mathrm{MHz}, \mathrm{CDCl}_{3}$

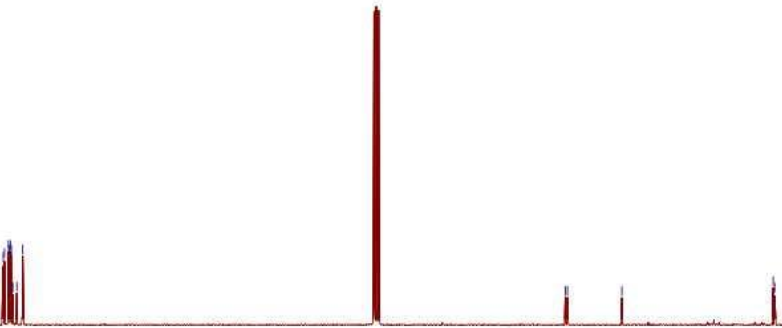

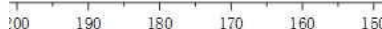
$10 \underset{f 1}{100}(\mathrm{ppm})$ 


\section{X-ray Crystal Structure and Data of 2d}

To get a high quality crystal for X-ray analysis, compound $\mathbf{2 d}$ was dissolved in ethyl acetate and hexane, and was allowed to crystalize via careful evaporation of the solvent. (CCDC: 2095006).

Figure S3. ORTEP drawings of 2d at 30\% displacement ellipsoid probability (the hydrogen atoms are omitted for clarity).

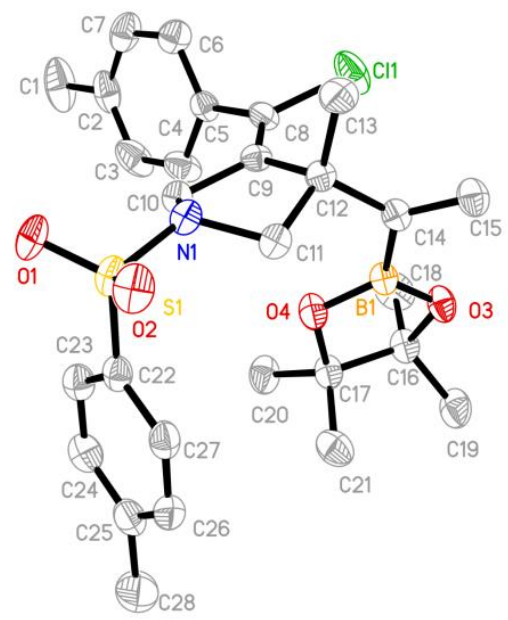

Table S1. Crystal data and structure refinement for $\mathbf{2 d .}$

\begin{tabular}{|l|l|}
\hline Empirical formula & $\mathrm{C}_{28} \mathrm{H}_{36} \mathrm{BClNO}_{4} \mathrm{~S}$ \\
\hline Formula weight & 528.90 \\
\hline Temperature & $293(2) \mathrm{K}$ \\
\hline Wavelength & $0.71073 \mathrm{~A}$ \\
\hline \multirow{2}{*}{ Unit cell dimensions } & $\mathrm{a}=10.584(2) \mathrm{A} \quad$ alpha $=90 \mathrm{deg}$. \\
\cline { 2 - 2 } & $\mathrm{b}=15.879(3) \mathrm{A} \quad$ beta $=91.38(3) \mathrm{deg}$. \\
\cline { 2 - 2 } & $\mathrm{c}=16.789(3) \mathrm{A} \quad$ gamma $=90 \mathrm{deg}$. \\
\hline Volume & $2820.7(10) \mathrm{A} \wedge$ \\
\hline Z, Calculated density & $4, \quad 1.245 \mathrm{Mg} / \mathrm{m}^{\wedge} 3$ \\
\hline Absorption coefficient & $0.243 \mathrm{~mm}{ }^{\wedge}-1$ \\
\hline F(000) & 1124 \\
\hline Theta range for data collection & 2.43 to $28.51 \mathrm{deg}$. \\
\hline Limiting indices & $-14<=\mathrm{h}<=14,-21<=\mathrm{k}<=15,-22<=1<=22$ \\
\hline Reflections collected / unique & $22943 / 7056[\mathrm{R}(\mathrm{int})=0.0660]$ \\
\hline
\end{tabular}




\begin{tabular}{|l|l|}
\hline Completeness to theta $=28.51$ & $98.6 \%$ \\
\hline Refinement method & Full-matrix least-squares on $\mathrm{F}^{\wedge} 2$ \\
\hline Data / restraints / parameters & $7056 / 0 / 326$ \\
\hline Goodness-of-fit on $\mathrm{F}^{\wedge} 2$ & 1.017 \\
\hline Final R indices [I>2sigma(I)] & $\mathrm{R} 1=0.0551, \mathrm{wR} 2=0.1574$ \\
\hline R indices (all data) & $\mathrm{R} 1=0.0996, \mathrm{wR} 2=0.1865$ \\
\hline Extinction coefficient & $0.0034(12)$ \\
\hline Largest diff. peak and hole & 0.413 and -0.366 e. $\mathrm{A}^{\wedge}-3$ \\
\hline
\end{tabular}




\section{X-ray Crystal Structure and Data of 4a}

To get a high quality crystal for X-ray analysis, compound $\mathbf{4 a}$ was dissolved in ethyl acetate and hexane, and was allowed to crystalize via careful evaporation of the solvent. (CCDC: 2095011).

Figure S3. ORTEP drawings of $\mathbf{4 a}$ at $30 \%$ displacement ellipsoid probability (the hydrogen atoms are omitted for clarity).

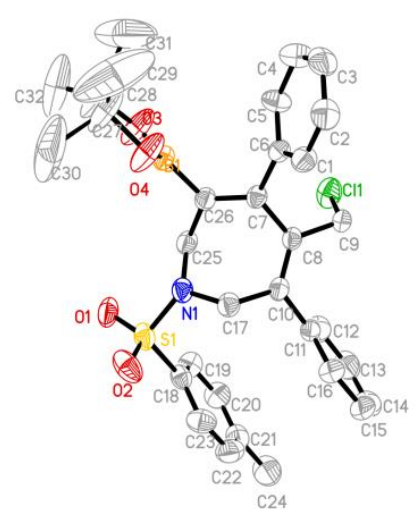

Table S2. Crystal data and structure refinement for $\mathbf{4 a}$.

\begin{tabular}{|l|l|}
\hline Empirical formula & $\mathrm{C}_{32} \mathrm{H}_{35} \mathrm{BClNO}_{4} \mathrm{~S}$ \\
\hline Formula weight & 575.93 \\
\hline Temperature & $293(2) \mathrm{K}$ \\
\hline Wavelength & $0.71073 \mathrm{~A}$ \\
\hline Crystal system, space group & triclinic, P-1 \\
\hline \multirow{2}{*}{ Unit cell dimensions } & $\mathrm{a}=10.971(2) \mathrm{A} \quad$ alpha $=109.29(3) \mathrm{deg}$. \\
\cline { 2 - 3 } & $\mathrm{b}=12.224(2) \mathrm{A} \quad$ beta $=91.45(3) \mathrm{deg}$. \\
\cline { 2 - 3 } & $\mathrm{c}=12.337(3) \mathrm{A} \quad$ gamma $=94.90(3) \mathrm{deg}$. \\
\hline Volume & $1553.6(5) \mathrm{A} \wedge 3$ \\
\hline Z, Calculated density & $2, \quad 1.231 \mathrm{Mg} / \mathrm{m}^{\wedge} 3$ \\
\hline Absorption coefficient & $0.226 \mathrm{~mm}^{\wedge}-1$ \\
\hline F(000) & 608 \\
\hline Crystal size & $0.20 \times 0.2 \times 0.20 \mathrm{~mm}$ \\
\hline Theta range for data collection & 1.87 to $28.48 \mathrm{deg}$. \\
\hline Limiting indices & $-11<=\mathrm{h}<=14,-16<=\mathrm{k}<=16,-16<=1<=16$ \\
\hline
\end{tabular}




\begin{tabular}{|l|l|}
\hline Reflections collected / unique & $12133 / 7565[\mathrm{R}(\mathrm{int})=0.1360]$ \\
\hline Completeness to theta $=28.48$ & $96.3 \%$ \\
\hline Refinement method & Full-matrix least-squares on $\mathrm{F}^{\wedge} 2$ \\
\hline Data / restraints / parameters & $7565 / 0 / 362$ \\
\hline Goodness-of-fit on $\mathrm{F}^{\wedge} 2$ & 0.977 \\
\hline Final R indices [I>2sigma(I)] & $\mathrm{R} 1=0.0959, \mathrm{wR} 2=0.2658$ \\
\hline R indices (all data) & $\mathrm{R} 1=0.2704, \mathrm{wR} 2=0.3784$ \\
\hline Absolute structure parameter & $0.004(4)$ \\
\hline Largest diff. peak and hole & 0.644 and -0.482 e. $\mathrm{A}^{\wedge}-3$ \\
\hline
\end{tabular}

